\title{
Structures magnétiques et polarimétrie neutronique
}

\author{
F. Tasset
}

Institut Laue-Langevin, BP. 156x, 38042 Grenoble cedex, France

\section{1-- Introduction}

Dans l'expérience pionnière de Shull et Smart sur MnO[1], la configuration des moments magnétiques dans la phase ordonnée a été déterminée à partir des intensités de diffraction de neutrons non polarisés. Souvent l'échantillon étudié est polycristallin et bien que cette technique fonctionne relativement bien pour des structures colinéaires, elle ne marche pas souvent pour une configuration plus compliquée, par exemple les structures hélimagnétiques non colinéaires et incommensurables. Pour de telles structures, même une étude par diffraction de neutrons sur des monocristaux de bonne qualité ne conduit pas à une solution unique. Pour des symétries cristallines d'ordre élevé, l'existence de plusieurs domaines magnétiques ajoute à la difficulté. Même la diffraction de neutrons polarisés avec analyse classique de la polarisation peut ne pas aboutir à une configuration de spin unique. [2]

Dans un tel contexte, la deuxième partie de ce cours introduit une technique récente et performante, la Polarimétrie Neutronique Sphérique $\{\mathrm{SNP}\}$, qui est en général capable de déterminer de manière unique la configuration de spin des structures modulées incommensurables ou celle des structures commensurables triangulaires.

Cette technique a été utilisée récemment pour mesurer des facteurs de forme antiferromagnétiques.

Tout cela repose sur la possibilité de mesurer les trois composantes, longitudinale et transverses, du vecteur de polarisation finale en utilisant Cryopad, un polarimètre en champ nul développé à l'ILL $[3,4]$. Cryopad-II est utilisé maintenant pour des mesures inélastiques plus difficiles mais passionnantes dans des systèmes magnétiques de basse dimensionnalité [5]. 


\section{1-Exemples de Structures magnétiques diverses}

terromagnetic

antif erromagnet ic

ferrimognetic

$\phi \phi \phi$

$\phi \phi \phi$

$\phi \phi \phi$
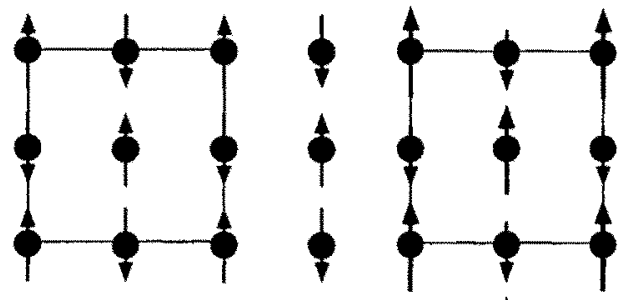

1

1999

(1.1.a)

19

(1.1.b)

$+\phi$

intanguter

canted

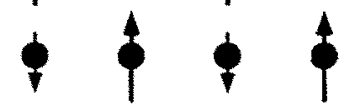

(1.1.c)

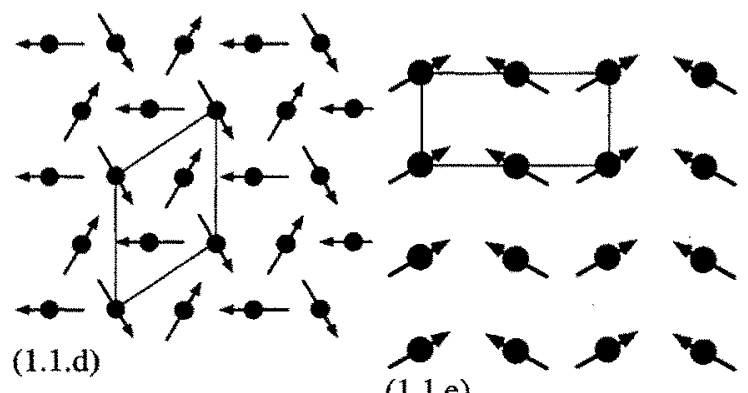

(1.1.e)

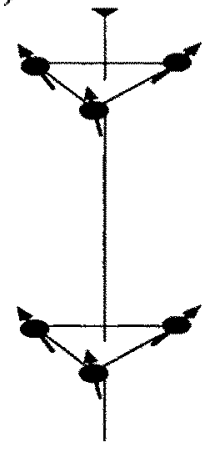

us b $m * 11 a$

(1.1.f)

$b \infty 6 \leftrightarrow$

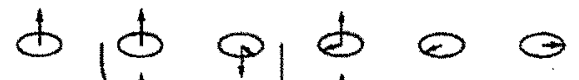

P. $\$ \oplus \oplus \Theta$

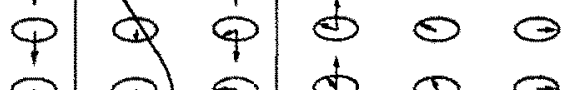

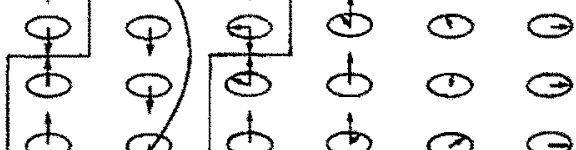

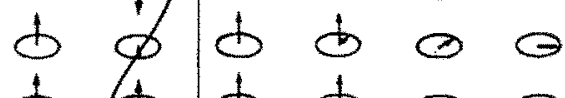

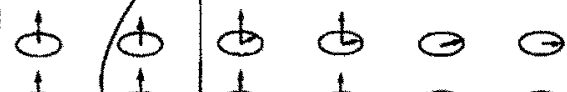

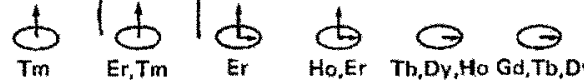

(a) (b) (c) (d) (a) (f)

(1.1.g)

Fig.1(a-g) Divers exemples de structures magnétiques ordonnées: 


\section{2- Le réseau de Bravais cristallin}

Les translations invariantes du réseau cristallin nucléaire sont données par le réseau de Bravais direct:

$$
\vec{l}=n_{1} \vec{a}+n_{2} \vec{b}+n_{3} \vec{c}
$$

où $\mathrm{n}_{1}, \mathrm{n}_{2}, \mathrm{n}_{3}$ sont des entiers

Les coordonnées d'un atome $\mathrm{j}$ quelconque dans la maille élémentaire sont données par :

$$
\vec{R}_{i j}=\vec{i}+\vec{r}_{j}
$$

avec $\quad \vec{r}_{j}=x_{j} \vec{a}+y_{j} \vec{b}+z_{j} \vec{c}$

et $\mathrm{x}, \mathrm{y}, \mathrm{z}$ inférieurs à 1 .

L' espace vectoriel dual appelé réseau réciproque est défini par

$$
\begin{aligned}
& \vec{a}^{*}=\frac{2 \pi}{v_{0}} \vec{b} \wedge \vec{c} \\
& \vec{b}^{*}=\frac{2 \pi}{v_{0}} \vec{c} \wedge \vec{a} \\
& \vec{c}^{*}=\frac{2 \pi}{v_{0}} \vec{a} \wedge \vec{b}
\end{aligned}
$$

dans lequel sont définis des vecteurs reliant l'origine à un nœud de l'espace réciproque

$$
\vec{\tau}=h \vec{a}^{*}+k \vec{b}^{*}+\vec{c}^{*}
$$

si $h, k$ et $l$ sont entiers ou à un point de la lère zone de Brillouin si

$$
\vec{k}=k_{1} \vec{a}^{*}+k_{2} \vec{b}^{*}+k_{3} \vec{c}^{*}
$$

\begin{tabular}{|c|c|c|}
\hline Espace Direct & & Espace réciproque \\
\hline $\begin{array}{l}\text { Pour un cristal péríodique } \\
\qquad \rho(\vec{r}+\vec{l})=\rho(\vec{r})\end{array}$ & (1.9) & $\begin{array}{l}\text { La transformée de Fourier de } \rho(\vec{r}) \text { est } \\
\text { discrète. (Elle est non nulle uniquement } \\
\text { aux nœuds du réseau réciproque) } \\
\qquad F(\vec{k})=\int \rho(\vec{r}) e^{\vec{k} f} d^{3} \vec{r}\end{array}$ \\
\hline
\end{tabular}

$\mathrm{k}_{1}, \mathrm{k}_{2}, \mathrm{k}_{3}$ sont inférieurs à l'unité.

\section{3- Cristallographie mathématique}

Les opérations mathématiques nécessaires pour décrire les structures magnétiques, leur stabilité et les sections efficaces de diffusion des neutrons sont résumées dans les tableaux suivants:

Tableau I: Densité continue périodique dans l'espace direct: 
Tableau 2: Distribution arbitraire aux nouds du réseau réciproque:

\begin{tabular}{|l|l|}
\hline Espace réciproque & Espace direct \\
\hline $\begin{array}{l}\text { Soit une distribution discrète arbitraire } \\
\text { des amplitudes } F(\vec{k})_{\text {sur les nœuds du }} \\
\text { réseau réciproque }\end{array}$ & $\begin{array}{l}\text { La transformation de Fourier inverse } \\
\text { donne une fonction périodique et } \\
\text { continue dans l'espace direct } \\
\rho(\vec{r}+\vec{l})=\rho(\vec{r})\end{array}$ \\
\hline
\end{tabular}

On peut donc établir le théorème suivant:

le réseau réciproque du réseau réciproque est le réseau direct.

Tableau 3: Des nouds du réseau direct vers le réseau réciproque:

\begin{tabular}{|c|c|}
\hline Espace direct & Espace réciproque \\
\hline $\begin{array}{l}\text { Soit une distribution arbitraire } \\
\text { d'amplitudes } m(\vec{l}) \text { aux nouds du réseau } \\
\text { réel. On peut l'écrire à l'aide de sa } \\
\text { transformée de Fourier inverse } \\
\qquad m(\vec{l})=\int \rho(\vec{k}) e^{-i \overrightarrow{k l}} d^{3} \vec{k}\end{array}$ & $\begin{array}{l}\text { La transformée de Fourier } \\
\qquad \rho(\vec{k})=\sum_{t} m(\vec{l}) e^{i \overrightarrow{k l}} \\
\text { est une fonction périodique et continue } \\
\text { dans lespace réciproque }\end{array}$ \\
\hline & $\rho(\vec{k}+\vec{K})=\rho(\vec{k})$ \\
\hline
\end{tabular}

Tableau 4: Généralisation vectorielle: au lieu de s'intéresser à une distribution scalaire $\mathrm{m}$, on part d'une distribution de vecteurs $\vec{m}$. Au lieu d'un seul réseau de Bravais on considère le cas de pjusieurs réseaux enchevêtrés repérés par l'indice j

\begin{tabular}{|l|l|}
\hline$\vec{m}_{j}(\vec{l})=\int \vec{\rho}_{j}(\vec{k}) e^{-i \vec{k} \vec{l}} d^{3} \vec{k} \quad(1.16)$ & $\vec{\rho}_{j}(\vec{k})=\sum_{i} \vec{m}_{j}(\vec{l}) e^{-i \vec{k}} \quad$ (1.17) \\
\hline $\begin{array}{l}\text { lintégrale est dans la première zone de } \\
\text { Brillouin seulement }\end{array}$ & \\
\hline
\end{tabular}

De cette façon on peut décomposer une distribution quelconque de moments magnétiques $\vec{m}_{j}$ dans un cristal

\section{4- Stabilité des structures magnétiques}

Les interactions entre les moments magnétiques peuvent en général se décrire en utilisant le Hamiltonien suivant

$$
H=-\sum_{\substack{b, l^{\prime} \\ j, j^{\prime}}} J_{u^{\prime} j j^{\prime}} \vec{m}_{j^{\prime}}^{\prime} \vec{m}_{l^{\prime} j^{\prime}}
$$

En refroidissant l'échantillon, les moments $\vec{m}_{l j}$ s'ordonnent éventuellement; les solutions $\vec{m}_{l j}$ doivent laisser le Hamiltonien invariant dans les translations de réseau.

La solution générale est :

$$
\vec{m}_{l j}=\int \vec{\rho}_{j}(\vec{k}) e^{-i \overrightarrow{k l}} d^{3} \vec{k}
$$

où $\vec{k}$ parcourt la première zone de Brillouin. 
En minimisant l'énergie du système on peut rencontrer plusieurs situations:

1) un seul vecteur $\vec{k}$ minimise l'énergie, l'état fondamental est alors:

$$
\vec{m}_{l, j}=\vec{m}_{j}^{k} e^{-\vec{k} t}
$$

Dans le cas où il y a plusieurs vecteurs $\vec{k}$ équivalents par symétrie le système peut choisir de rester dans une configuration multi $\vec{k}$

$$
\vec{m}_{l, j}=\sum_{e t h i l e d e \vec{k}} \vec{m}_{j}^{\vec{k}} e^{-i \overrightarrow{k l}}
$$

2) un vecteur $\vec{k}$ et ses harmoniques sont favorables

$$
\vec{m}_{l_{i}, j}=\sum_{\text {harmoniques }} \vec{m}_{j}^{\vec{k}} e^{-i \vec{k}}
$$

Si il y a plusieurs vecteurs $\vec{k}$ (une étoile de $\vec{k}$ ) équivalents on pourra avoir les harmoniques croisées aussi appelées intermodulations

\section{5- Représentation dans l'espace direct}

Pour simplifier la représentation nous allons supposer qu'il n'y a qu'un seul atome par maille; Ceci veut dire qu'il n'y a qu'un réseau, $\mathrm{j}=1$.

$$
\begin{aligned}
& \vec{m}_{l}=\sum_{\vec{k}} \vec{m}^{\vec{k}} e^{-i \overrightarrow{k l}} \\
& \overrightarrow{k l}=2 \pi\left(n_{1} k_{1}+n_{2} k_{2}+n_{3} k_{3}\right)
\end{aligned}
$$

Il faut distinguer 2 cas : $\overrightarrow{k l}$ est, ou n'est pas, multiple de $\pi$.

$\vec{m}_{l}$.est forcément réel. Si $\overrightarrow{k l}$ n'est pas multiple de $\pi$ alors pour tout $\vec{k}$ il faut associer $-\vec{k}$ qui n'est pas équivalent par translation de réseau. Par ailleurs

et

$$
\begin{aligned}
& \vec{m}^{-\vec{k}}=\left(\vec{m}^{\vec{k}}\right)^{*} \\
& m_{l}=\left|\sum_{k}\left(\vec{m}^{k} e^{-i \vec{k} l}+\left(\vec{m}^{k}\right)^{*} e^{i \vec{k} l}\right)\right|=2\left|\vec{m}^{\vec{k}}\right| \cos (k l+\varphi)
\end{aligned}
$$

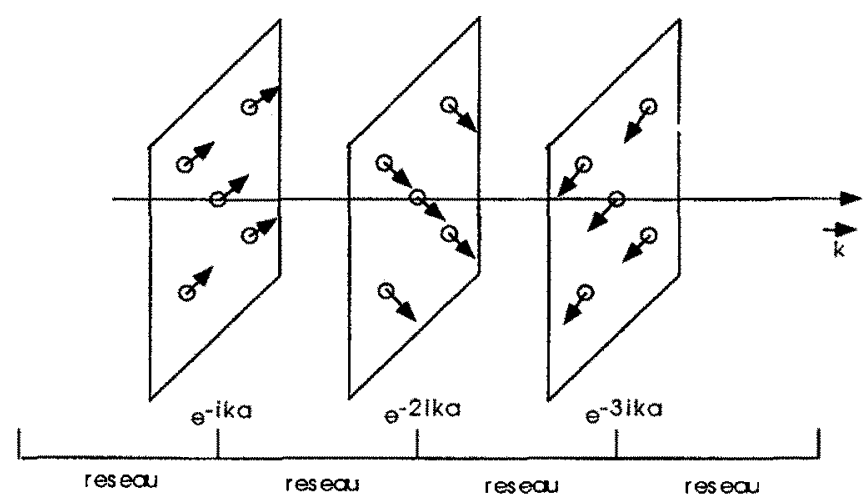

les plans perpendiculares à $\mathrm{k}$ contiennent les moments de mëme drection

Fig. 2 :les moments magnétiques contenus dans des plans perpendiculaires à $k$ sont parallèles. 


\section{6- Recherche du vecteur de propagation}

Les raies magnétiques observées par différence des diagrammes en dessous et en dessus de la température d'ordre vont révéler la longueur de $\vec{Q}=\vec{\tau} \pm \vec{k}$

L'identification peut être intuitive si le module de $\mathrm{k}$ est faible (modulation à longue période) et le diagramme peu encombré de pics (groupes d'espaces très symétriques). Les satellites apparaîtront comme attachés à un noud du réseau réciproque, on parlera par exemple des satellites de l'origine qui apparaissent comme des raies intenses aux petits angles.

Dans ce cas on pourra utiliser une méthode graphique simple comme c'est illustré dans la figure suivante.

Les cercles tracés sont de rayon

$$
H_{i}=\frac{4 \pi \sin \theta_{i}}{\lambda}
$$

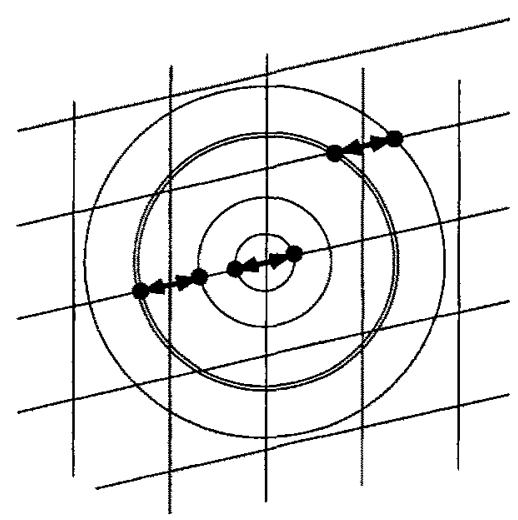

Fig. 3 : A la recherche du vecteur de propagation

La méthode la plus efficace est de confier à un ordinateur la liste des longueurs mesurées pour $\mathrm{Q}$ ainsi qu'une description du réseau réciproque afin qu'il recherche systématiquement les vecteurs $\mathrm{k}$ qui peuvent les satisfaire. La zone de Brillouin est découpée en une fine grille de $\vec{k}$ et pour chaque $\vec{\tau}$ on calcule tous les $\vec{Q}=\vec{\tau} \pm \vec{k}_{i}$. On compare alors $\left|\vec{Q}_{\text {cal }}\right|$ et $H_{i} \pm \Delta H_{i}$ 

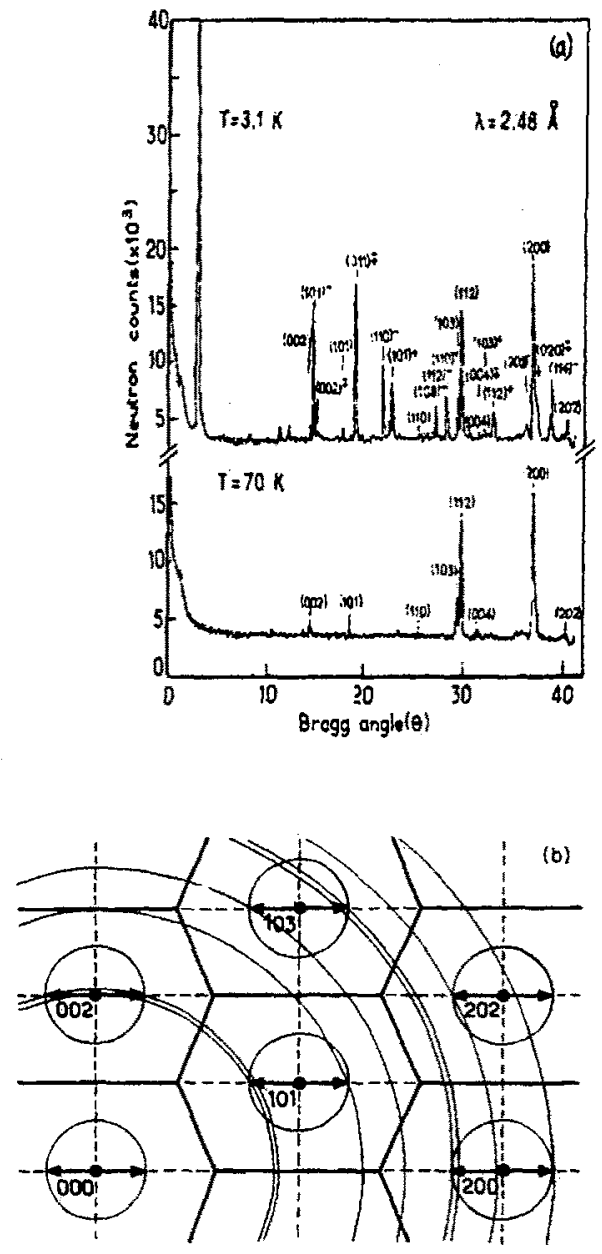

Fig. 4 : Vecteur de propagation de $\mathrm{TbR} \mathrm{H}_{2} \mathrm{Si}_{2}$

TbRu2Si2 adopte une structure quadratique avec un groupe d'espace $14 / \mathrm{mmm}$

a) Les diagrammes de diffraction sur poudre ont été enregistrés de part et d'autre de la transition à $T_{N}=55 K$

b) Le réseau réciproque et les zones de Brillouin associées. On voit que l'ordre magnétique correspond à $k=\langle 0.232,0,0\rangle$ 


\section{2-- Les bases de la diffusion des neutrons}

Quand un neutron rencontre un atome magnétique, il est soumis à deux potentiels de diffusion :

1) une diffusion nucléaire qui révèle l'existence et la nature du noyau

2) une diffusion magnétique, provenant principalement des électrons non appariés.

\subsection{Diffusion nucléaire cohérente}

L'opérateur représentant la diffusion nucléaire sur le noyau de l'atome j s'écrit :

$$
a_{N j}=b_{j}+A_{j} \vec{\sigma} \cdot \vec{I}_{j}
$$

$\vec{I}_{j}$ est le vecteur opérateur de spin nucléaire. A température ambiante, le moment angulaire de spin est orienté aléatoirement d'un site à l'autre. Lorsqu'on somme sur les sites pour obtenir l'amplitude cohérente totale sa contribution s'annule (elle est incohérente). La diffusion moyenne cohérente qui nous intéresse ici est donc $a_{n j}=b_{j}$ que nous appellerons la longueur de diffusion ou longueur de Fermi. Elle est souvent exprimée en unité $10^{-12} \mathrm{~cm}$, mais plus récemment en $\mathrm{fm}\left(1 \mathrm{fm}=10^{-15} \mathrm{~m}, 10\right.$ fois plus petit).

La section efficace nucléaire cohérente pour tous les atomes du cristal est

$$
\left(\frac{d \sigma}{d \Omega}\right)_{N}=\left|\sum_{i j} b_{j} e^{i \vec{Q} \vec{R}_{i j}}\right|^{2}=N \frac{(2 \pi)^{3}}{v_{0}} \sum_{\tau}\left|F_{N}\right|^{2} \delta(\vec{Q}-\vec{\tau})
$$

avec

$$
F_{N}(\vec{Q})=\sum_{j} b e^{i \vec{Q} \vec{q}_{i}} e^{-W_{j}}
$$

N.B. il faut bien comprendre que $A_{j} \vec{\sigma}, \vec{I}_{j}$ n'est pas l'interaction qui nous intéresse pour le magnétisme électronique, il s'agit de la partie de la force d'interaction nucléaire forte qui est responsable de la diffusion du neutron sur le noyau et dépend fortement du spin nucléaire. Evidemment si la polarisation de spin nucléaire n'est pas nulle il faut revoir les expressions.

\subsection{Amplitude de diffusion magnétique}

$$
\begin{aligned}
& a_{M j}=p \vec{\sigma} \cdot \vec{M}_{j 1}(\vec{Q}) \\
& p=\frac{\gamma e^{2}}{2 m_{e} c^{2}}=\frac{\gamma r_{0}}{2}=0.269610^{-12} \mathrm{~cm} / \mu_{B}
\end{aligned}
$$

$\vec{M}(\vec{Q})$ est la composante de Fourier de la densité magnétique $\vec{M}(\vec{r})$ pour le vecteur d'onde $\vec{Q}$ et $\overrightarrow{M_{1}}(\vec{Q})$ est la projection de $\vec{M}(\vec{Q})$ sur le plan perpendiculaire à $\vec{Q}$

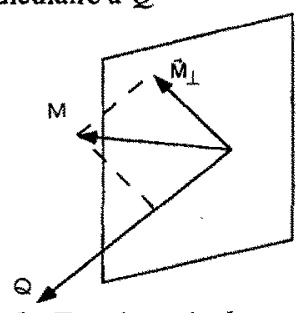

Fig. 5 : Projection de la composante de Fourier résultant de la nature dipolaire de l'interaction (vue dans l'espace réciproque) 
On peut montrer que

$$
\begin{array}{cc} 
& \vec{M}_{\perp}=\hat{Q} \wedge(\vec{M} \wedge \hat{Q})=\vec{M}-\hat{Q}(\vec{M} \cdot \hat{Q}) \\
\text { Avec } & \hat{Q}=\text { vecteur unitaire }=\frac{\vec{Q}}{Q} \\
\text { et } & \vec{M}(\bar{Q})=\vec{m} f(\vec{Q})
\end{array}
$$

\subsection{Les deux sections efficaces de diffusion des neutrons}

Table 5 : Les sections efficaces de diffusion et les graphes correspondants.

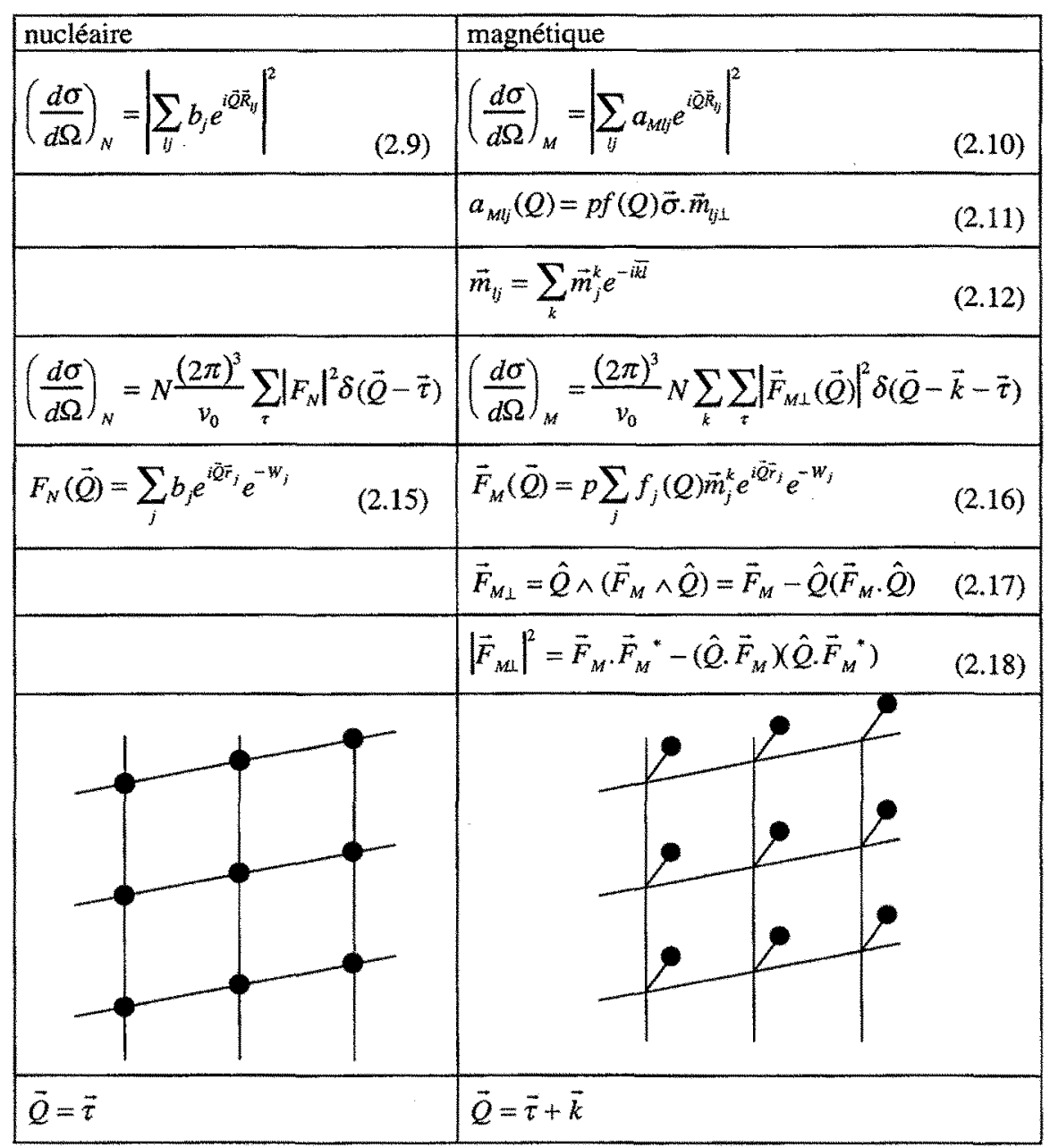




\subsection{Différentes types de section efficaces de diffusion magnétiques}

Table 6: Représentation graphique de diverses Sections efficaces dans l'espace réciproque

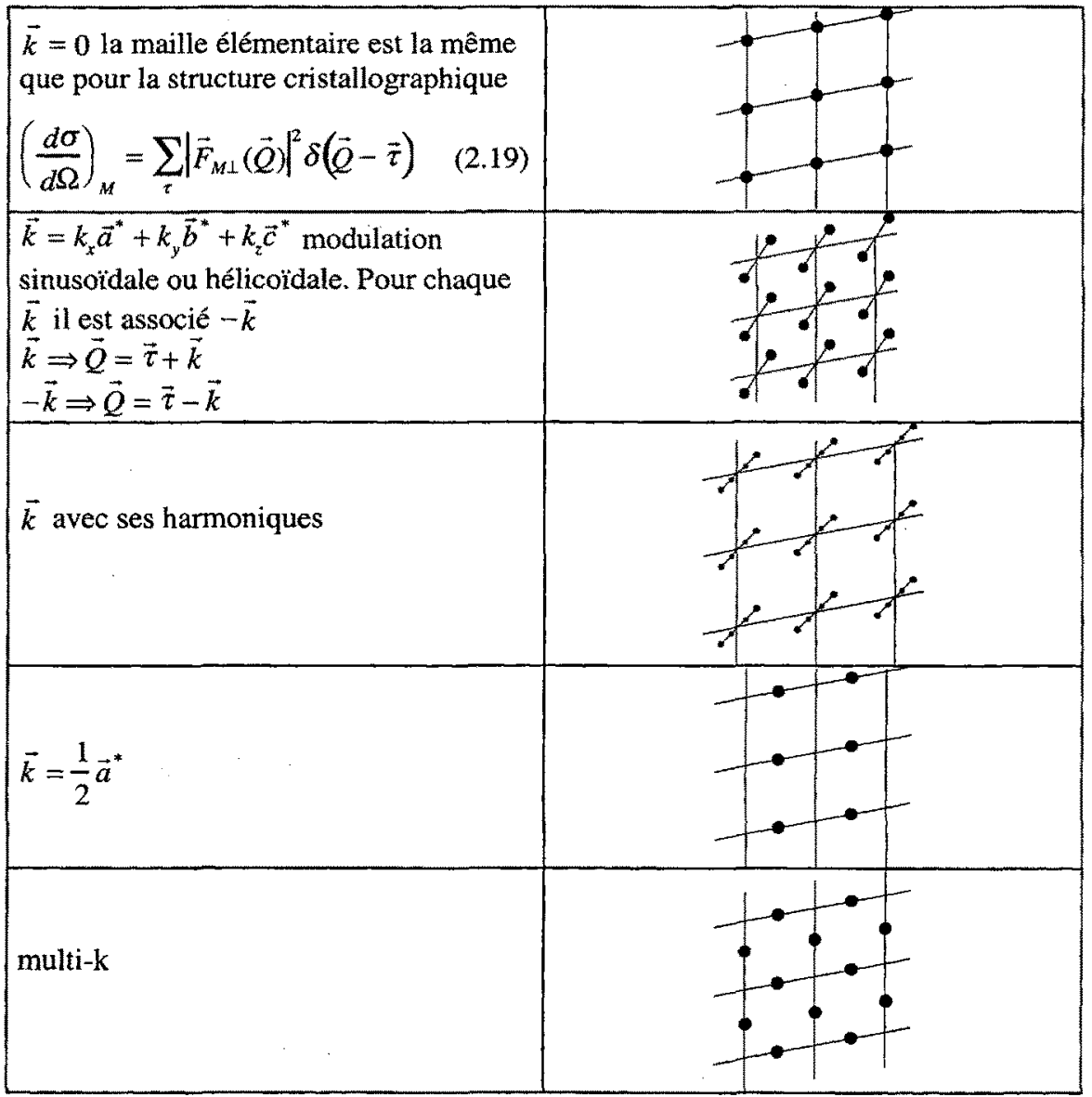




\subsection{Instrumentation}

2.5.1-- Diffractomètres à deux axes pour poudre

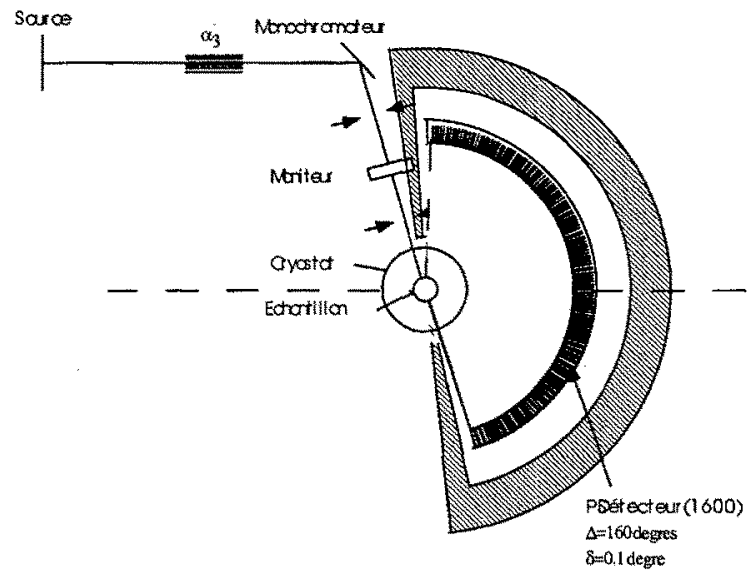

Le Diffroctomet te a poude Dzo de I'LL

Fig. 6 : Le diffractomètre D20 de l'ILL: un grand multidétecteur installé sur un faisceau à haut flux.

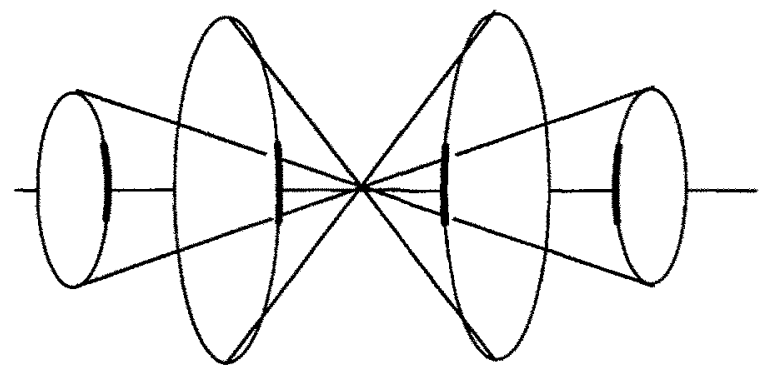

Fig. 7 : Les cônes Debye Scherrer produits par une poudre (vue de côtê)

$$
I_{\mathrm{int}}=\Delta(2 \theta) \sum_{i} J\left(2 \theta_{i}\right)=\int \frac{d \sigma}{d \Omega} d \Omega=\frac{N}{v_{0}} \frac{\lambda^{3}}{8 \pi \sin \theta \sin 2 \theta} \frac{l}{r} \sum\left|F^{2}\right| A
$$

le facteur de Lorentz $\frac{1}{\sin \theta \sin 2 \theta}$

résulte de la probabilité pour un cristallite d'être orienté dans la direction appropriée multipliée par la portion de cône interceptée par le détecteur. 


\subsection{2- Diffractometre pour monocristaux}

\subsubsection{1-Première méthode, le 4 cercles}

En jouant sur les 4 cercles du diffractomètre, le vecteur réciproque $\vec{\tau}$ est amené à traverser la sphère d'Ewald dans le plan horizontal. Le plan de diffusion est horizontal et le détecteur est placé à l'angle de Bragg approprié. De nos jours on utilise souvent un petit détecteur à localisation, très utile pour visualiser la forme de la section efficace (détection éventuelle de satellites, etc.)

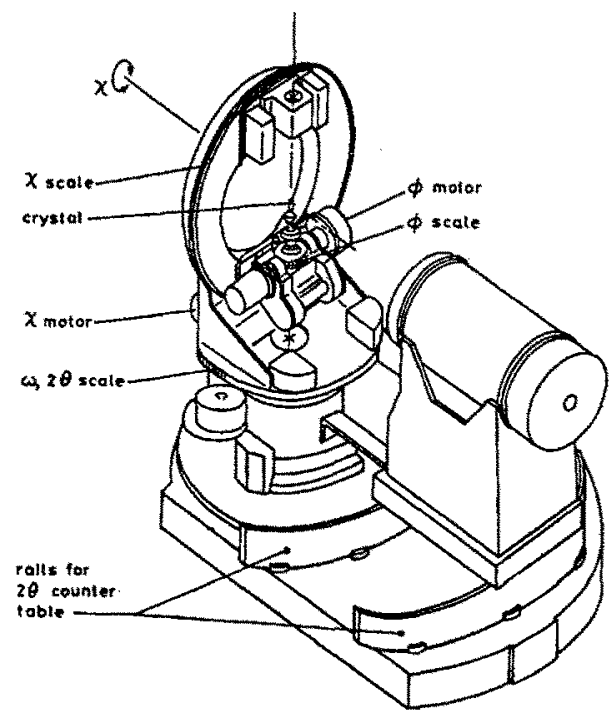

\begin{tabular}{|l|l|}
\hline \multicolumn{2}{|l|}{ Appellation des 4 cercles } \\
\hline$\omega$ & Cristal \\
\hline$\chi$ & Cristal \\
\hline$\varphi$ & Cristal \\
\hline$\chi$ or $2 \theta$ & Détecteur \\
\hline
\end{tabular}

Fig. 8: Un diffractomètre 4 cercles 


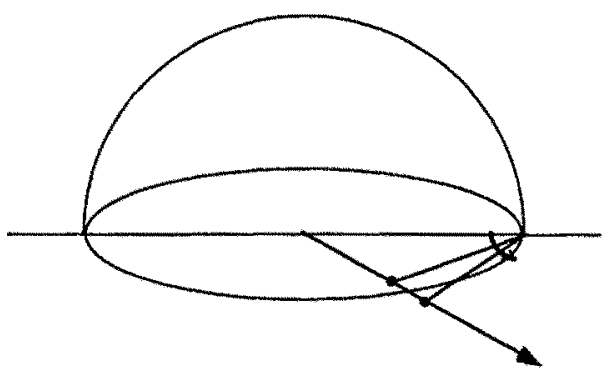

Fig. 9: Les intensités de diffraction sont mesurées dans le plan horizontal en tournant le cristal échantillon. Le noud du réseau réciproque correspondant traverse la sphère d'Ewald.

En toumant le cristal avec l'angle $\omega$, le vecteur $\vec{\tau}$ est amené à traverser la sphère d'Ewald. On obtient ainsi une courbe de réflexion qui est intégrée par le détecteur.

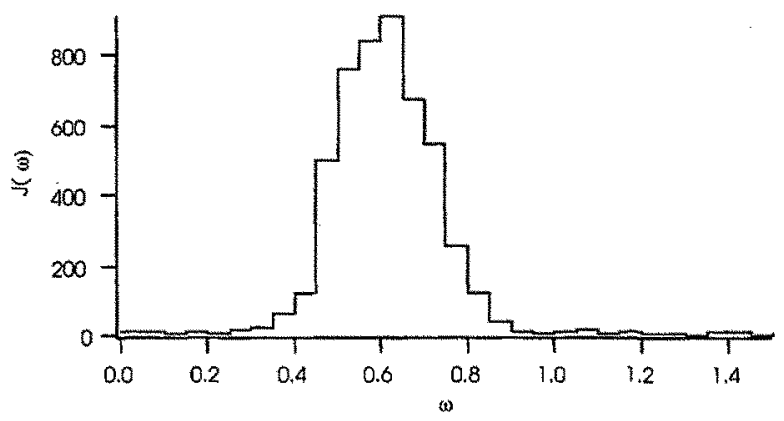

Fig. 10: Histogramme du nombre de neutrons détectés pendant la rotation pas à pas (i.e. un scan de neutron)

$$
I_{\text {iat }}=\Delta(\omega) \sum_{i} J\left(\omega_{i}\right)=\int \frac{d \sigma}{d \Omega} d \Omega=\frac{N}{v_{0}} \frac{\lambda^{3}}{\sin 2 \theta}\left|F^{2}\right|_{A}
$$

le facteur de Lorentz correspondant est $\frac{1}{\sin 2 \theta}$ 


\subsubsection{2- La méthode "normal beam" avec détecteur incliné}

Par analogie avec la méthode du "cristal tournant" utilisée avec les rayons $\mathrm{x}$ il existe une géométrie alternative où l'on garde toujours le même axe du cristal vertical et l'on incline le détecteur pour recueillir les neutrons diffractés hors du plan horizontal. Cette méthode est utilisée lorsqu'on ne peut pas incliner facilement les gros cryostats à Hélium liquide contenant les bobines supraconductrices nécessaires à l'application d'un champ magnétique intense sur l'échantillon (voir la description de D3 dans la section 4.2)

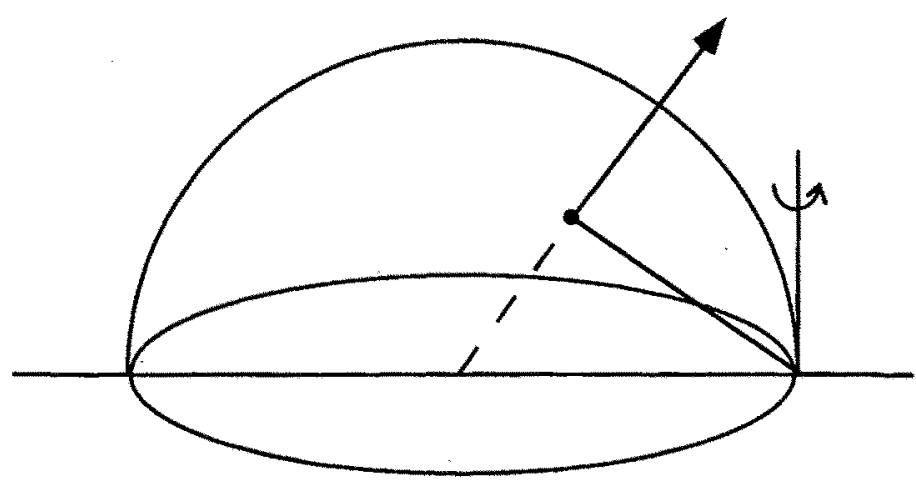

\begin{tabular}{|l|l|}
\hline \multicolumn{2}{|l|}{$\begin{array}{l}\text { Les } 3 \text { angles de la } \\
\text { géométrie "normal beam" }\end{array}$} \\
\hline$\omega$ & cristal \\
\hline$y$ & détecteur \\
\hline$v$ & $\begin{array}{l}\text { inclinaison du } \\
\text { détecteur }\end{array}$ \\
\hline
\end{tabular}

Fig. 11: Géométrie « normal beam »: le nceud du réseau réciproque traverse la sphère d'Ewald en dehors du plan horizontal. Le détecteur doit être incliné pour intercepter les neutrons diffusés. En baissant le détecteur on libère plus d'espace au-dessus de l'échantillon (cf. Nouvel arrangement 10 Tesla sur D3).

En tournant le cristal autour de l'axe vertical avec l'angle $\omega, \vec{\tau}$ traverse la sphère d'Ewald. Sauf pour la strate zéro de l'espace réciproque les réflexions ne sont pas dans le plan horizontal. Le détecteur doit être incliné pour intercepter les neutròns diffusés.

$$
I_{\text {int }}=\Delta(\omega) \sum_{i} J\left(\omega_{i}\right)=\int \frac{d \sigma}{d \Omega} d \Omega=\frac{N}{v_{0}} \frac{\lambda^{3}}{\sin \gamma \sin v}\left|F^{2}\right| A
$$

Pour cette géométrie, le facteur de Lorentz est $\frac{1}{\sin \gamma \sin v}$ 


\subsubsection{Etude des moments magnétiques, module et direction}

A cause de la nature dipolaire de l'interaction magnétique du neutron, l'amplitude de diffusion magnétique comprend 3 composantes qui se transforment comme un vecteur.

Elle a pris le nom de vecteur interaction magnétique $\vec{M}=p \vec{F}_{M \perp}$.

Fig. 12 : Le vecteur interaction magnétique

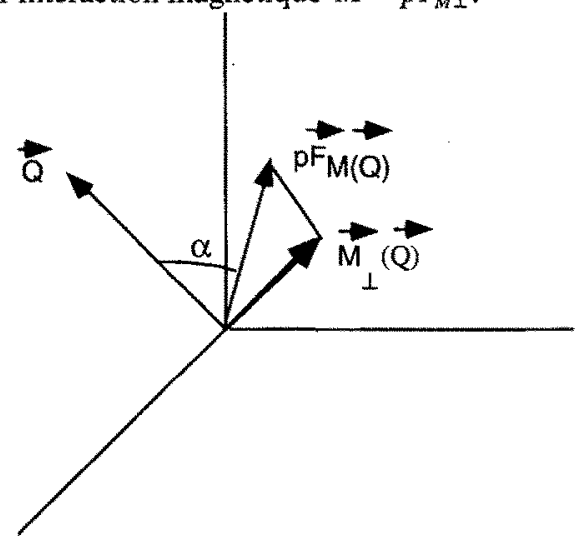

Notons que

$$
\left|\vec{m}_{\perp}\right|=m \sin \alpha
$$

et que

$$
\vec{F}_{M L}(\vec{Q})=\sum_{j} p_{j} f_{j}(\vec{Q}) \vec{m}_{j \perp}^{k} e^{i \vec{Q}_{j}}
$$

Remarquons que pour une réflexion magnétique sur une structure ordonnée colinéaire telle que $\vec{m} / / \vec{Q}, \alpha=0$, le vecteur d'interaction magnétique s'annule car tous les $\vec{m}_{j}^{k}$ sont nuls. La réciproque étant que la connaissance du vecteur d'interaction magnétique pour une seule réflexion ne dit rien sur la composante selon $\vec{Q}$ du facteur de structure magnétique correspondant (le neutron ne voit que 2 des 3 composantes)

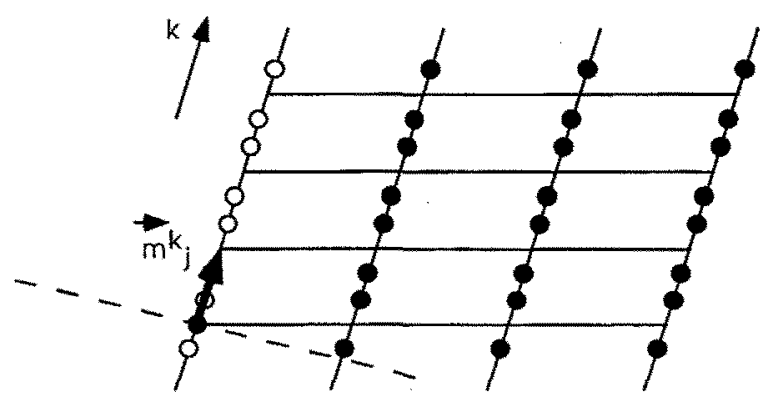

Fig. 13: Annulation de la diffusion magnétique due à la nature dipolaire de l'interaction (les cercles vides correspondent à des intensités nulles, par suite de l'orientation colinéaire du vecteur interaction magnétique et du vecteur de diffusion du neutron) 
Possible Indétermination dans la direction des moments magnétiques.

Supposons que la structure magnétique soit colinéaire et que nous soyons en train de chercher la direction des moments magnétiques sur la base des intensités observées dans un diagramme de poudre.

$$
\left|\vec{F}_{M L}(Q)\right|=\sin \alpha\left|\sum_{j} \pm p_{j} f_{j}(Q) \backslash m_{j}^{k}\right| e^{i \bar{Q} \bar{r}_{j}} \mid
$$

L'intensité sur un pic donné résulte de toutes les réflexions ayant le même module pour $Q$ puisqu'elles diffractent en même temps au même angle $2 \vartheta$. Toutes ces réflexions peuvent avoir des facteurs de structure identiques ou non

$$
\left|\sum_{j} \pm p_{j} f_{j}(Q)\right| m_{j}^{k}\left|e^{i \bar{Q} r_{j}}\right|
$$

mais elles ont des $\sin \alpha$ différents (i.e. leurs vecteurs d'interaction magnétique sont différents). Par suite

$$
I \propto \sum_{\text {Q non equivalent }} z_{Q}\left(\sin ^{2} \alpha\right\rangle\left|\sum_{j} \pm p_{j} f_{j}(Q)\right| m_{j}^{k}\left|e^{i \vec{Q} \vec{Q}_{j}}\right|^{2} .
$$

Shirane a montré que pour la symétrie cubique $\left\langle\sin ^{2} \alpha\right\rangle=2 / 3$ indépendamment de la direction du moment magnétique et que pour une symétrie uniaxe l'intensité dépend de $\theta$ mais pas de $\varphi$.

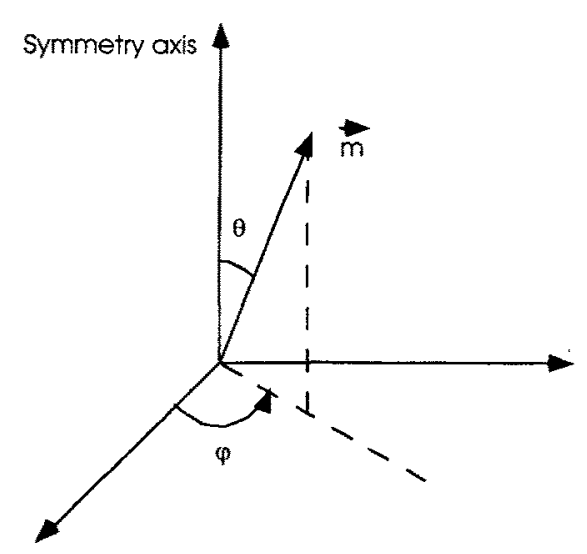

Fig. 14: Définition des angles polaires par rapport à l'axe principal de symétrie 


\subsubsection{Domaines Magnétiques}

\subsubsection{1- Les domaines $K$}

Quand il s'ordonne magnétiquement, le système choisit le vecteur $\mathrm{K}$ qui minimise l'énergie. Mais plusieurs $\mathrm{K}$ peuvent donner la même énergie par suite de la symétrie cristalline. Une partie du cristal va choisir $k_{1}$ pendant que d'autres parties choisissent $k_{2}, k_{3}$, etc. Le célèbre exemple de $\mathrm{MnO}$ avec ses 4 directions d'axes ternaires dans le système cubique est représenté ici :

$$
\vec{k}_{1}=\left(\frac{1}{2}, \frac{1}{2}, \frac{1}{2}\right) ; \vec{k}_{2}=\left(\frac{\overline{1}}{2}, \frac{1}{2}, \frac{1}{2}\right) ; \vec{k}_{3}=\left(\frac{1}{2}, \frac{\overline{1}}{2}, \frac{1}{2}\right) ; \vec{k}_{4}=\left(\frac{1}{2}, \frac{1}{2}, \frac{\overline{1}}{2}\right)
$$
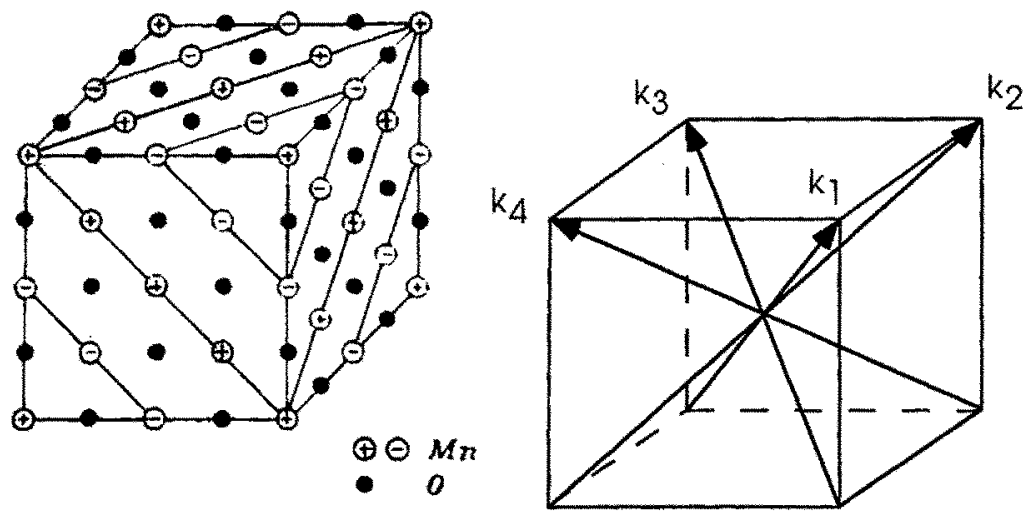

Fig. 15 : Les 4 domaines $k$ dans $\mathrm{MnO}$

Ces directions différentes peuvent être distinctes dans l'espace réciproque ou rendues indiscernables par suite des symétries de translations de réseau comme on le voit dans l'exemple suivant

$$
\begin{aligned}
& \vec{k}_{1}=\left(\frac{1}{2}, 0,0\right) \\
& \vec{k}_{2}=\left(0, \frac{1}{2}, 0\right)
\end{aligned}
$$

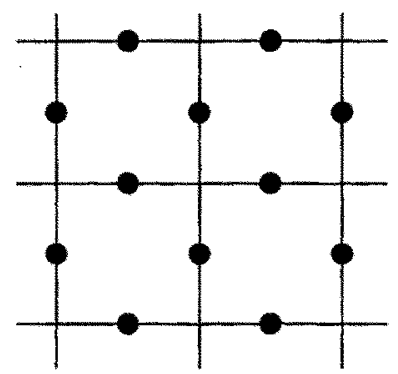

Fig. 16: Superposition de $2 \mathrm{k}$ par suite des translations de réseau 


\subsubsection{2- Les domaines $S$}

Il existe une autre sorte de domaines magnétiques: ils sont dus aux symétries du site cristallographique sur lequel s'ordonne le moment magnétique. De tels domaines s'appellent des domaines $S$ (S pour Spin).

A l'intérieur d'un domaine $\mathrm{K}$, on peut trouver que les moments magnétiques $\vec{m}_{j}^{*}$ ont une seule direction possible ou alors plusieurs directions équivalentes par rapport aux axes $\mathrm{a}, \mathrm{b}, \mathrm{c}$ et la direction $\mathrm{k}$.

On peut montrer que, par suite de l'existence des domaines $S$, les conclusions de Shirane concernant les limitations rencontrées dans la détermination des directions de moments magnétiques à partir des intensités de diffraction des neutrons sur poudre subsistent lors des études sur monocristaux dès lors que les proportions de domaines $\mathrm{S}$ sont égales. Pour résoudre les ambiguittés il est nécessaire de déséquilibrer les proportions de domaines. Ceci peut se faire en appliquant une contrainte extérieure uniaxiale ou un champ magnétique ou électrique.

\subsection{5- Orientation relative des moments magnétiques}

Nous avons vu l'effet

-du vecteur de propagation

-de la direction du moment magnétique

Il nous reste à voir l'effet de l'orientation relative des moments magnétiques lorsqu'il y a plus d'un atome magnétique dans ma maille élémentaire :

$$
\begin{gathered}
I \propto\left|\vec{F}_{M \perp}\right|^{2}=\vec{F}_{M} \vec{F}_{M}^{*}-\left(\hat{Q} \vec{F}_{M}\right)\left(\hat{Q}_{F_{M}}^{*}\right) \\
\vec{F}_{M}(\vec{Q})=p \sum_{j} f_{j}(\vec{Q}) \vec{m}_{j}^{k} e^{i \vec{Q} \vec{r}_{j}}
\end{gathered}
$$

Il faut faire attention de bien ajouter les amplitudes en provenance des différents atomes dans la maille élémentaire.

Tout d'abord, pour un site cristallographique donné, les positions qui sont reliées par des éléments de symétrie tels que

Rotations

Inversions

Rotation + translation fractionnaire

En second lieu, pour les différents sites cristallographiques. 


\section{Interactions magnétiques}

$\mathrm{Si}$, dans l'espace réel, les interactions doivent être sommées sur tous les atomes du cristal

$$
H=-\sum_{i j} \sum_{i^{\prime}} J_{\left.i j^{\prime}\right\}^{\prime}} \vec{m}_{i j} \vec{m}_{l^{\prime} j^{\prime}}
$$

dans l'espace réciproque

$$
H=-\sum_{\vec{k}} \sum_{j j^{\prime}} J_{j j^{\prime}}(\vec{k}) \vec{m}_{j}^{k} \vec{m}_{j^{*}}^{-k}
$$

les interactions ne doivent plus qu'être sommées sur les atomes de la maille.

En effet

$$
\vec{m}_{i j}=\sum_{k} \vec{m}_{j}^{k} e^{-\sqrt{k g}} \text { et } \vec{m}_{i^{\prime}}=\sum_{k^{\prime}} \vec{m}_{j^{\prime}}^{k^{\prime}} e^{-i \bar{k}^{\prime} \hat{T}^{\prime}}
$$

alors

$$
H=-\sum_{i l^{\prime}} \sum_{i j^{\prime}} J_{1 l^{\prime} j j^{\prime}} \sum_{k k^{\prime}} \vec{m}_{j}^{k} e^{-i \vec{k} \vec{t}} \vec{m}_{j^{\prime}}^{k^{\prime}} e^{-i \vec{k} \vec{q}^{\prime}}
$$

Introduisons

$$
e^{-i \overrightarrow{k I}} e^{i k \bar{k}^{*}}=1
$$

alors

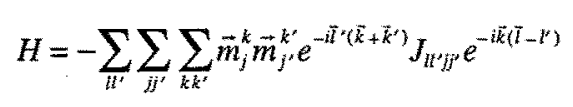

posons par définition

$$
J_{i j j^{\prime}}(k)=\sum_{i} J_{i^{\prime j j}} e^{-\vec{k}(\bar{l}(-\vec{I})} .
$$

et notons que cette transformée de Fourier est indépendante de $l^{\prime}$

Alors

$$
\begin{gathered}
H=-\sum_{i l^{\prime}} \sum_{k k^{\prime}} \vec{m}_{j}^{k} \vec{m}_{j^{\prime}}^{k^{\prime}} J_{j j^{\prime}}(\vec{k}) \sum_{l^{\prime}} e^{-i l^{\prime}(\vec{k}+\vec{k})} \\
\sum_{l^{\prime}} e^{-\vec{i}^{\prime}\left(\vec{k}+\vec{k}^{\prime}\right)}=\delta\left(\vec{k}+\vec{k}^{\prime}-\vec{\tau}\right)
\end{gathered}
$$

mais dans la première zone de Brillouin $\vec{\tau}=0$ et donc $\vec{k}^{\prime}=-\vec{k}$.

On trouve bien l'expression recherchée

$$
H=-\sum_{\vec{k}} \sum_{j j^{\prime}} J_{j j^{\prime}}(\vec{k}) \vec{m}_{j}^{k} \vec{m}_{j^{\prime}}^{-k}
$$




\section{1- Classification des structures magnétiques}

Prenons le cas général où le vecteur $\vec{k}$ est un point sans symétrie particulière dans la zone de Brillouin. On dit que la structure est incommensurable car $\vec{k} \vec{l}$ n'est pas un multiple de $2 \pi$. Exemple : la structure sinusoïdale :

$$
\vec{m}_{l j}=\vec{m}_{j}^{k} e^{-i \overrightarrow{k l}}+\vec{m}_{j}^{-k} e^{i \vec{k} t}=\vec{u}_{j} \cos \left(\vec{k} \vec{l}+\varphi_{j}\right)
$$

La longueur des moments magnétiques va changer d'une maille à la suivante

$$
\vec{m}_{j}^{k}=\frac{\vec{u}_{i}}{2} e^{i \varphi_{j}}
$$

$$
\vec{F}_{M}(Q)=p \sum_{j} f_{j}(Q) \frac{\vec{u}_{i}}{2} e^{i\left(\hat{Q}, \vec{r}_{j}+\varphi_{j}\right)}
$$

Fig. 17 : Structure magnétique sinusoüdale

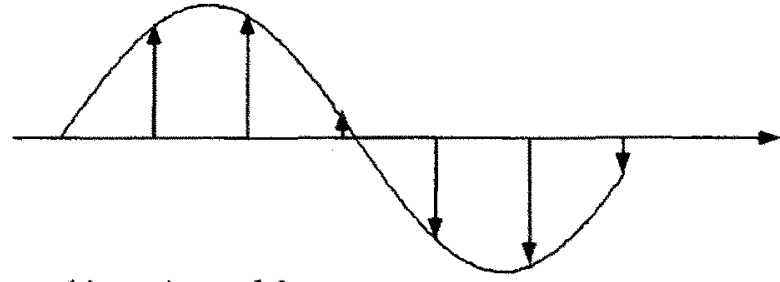

\subsection{1- Structure carrée}

Parfois, à basse température, la modulation change de forme, elle devient carrée.

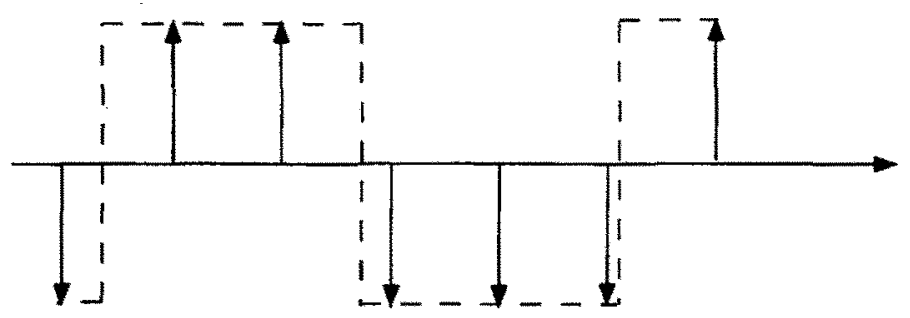

\section{Fig.: 18 Structure carrée}

La structure est toujours incommensurable, mais la longueur des moments magnétiques ne change pas, seulement le signe.

La décomposition en composantes de Fourier s'écrit

$$
\vec{m}_{b j}=\sum_{n} \vec{m}^{n k} e^{-i n k \vec{k}}
$$

avec une infinité de vecteurs de propagation $n \vec{k}$, avec n entier.

$$
\begin{aligned}
& \vec{m}_{j}^{n k}=\frac{2 m}{i \pi n} e^{i \varphi_{j}} \vec{u} \\
& \vec{m}_{j}^{k}=\frac{2 m}{i \pi} e^{i \varphi_{j}} \vec{u}
\end{aligned}
$$




$$
\begin{aligned}
& \vec{m}_{j}^{3 k}=\frac{2 m}{3 i \pi} e^{3 i \varphi_{j}} \vec{u} \\
& \vec{F}_{M}\left(\vec{Q}^{n}\right)=\sum_{j} f_{j}(Q) \frac{2 m_{i \pi n}}{i \pi n} e^{i\left(\vec{Q}_{j_{j}}+n \varphi_{j}\right)}
\end{aligned}
$$

S'il n'y a qu'un atome par maille alors

$$
\begin{aligned}
& \frac{F_{M}\left(Q^{3}\right)}{F_{M}\left(Q^{1}\right)}=\frac{1}{3} \frac{f\left(Q^{3}\right)}{f\left(Q^{1}\right)} \\
& \frac{I_{M}\left(Q^{3}\right)}{I_{M}\left(Q^{1}\right)} \cong \frac{1}{9}
\end{aligned}
$$

\subsection{2- Structures hélicoïdales}

Pour les structures hélicoidales, les moments magnétiques se décrivent à l'aide de deux vecteurs

$$
\vec{m}_{l j}=\vec{u}_{j} \cos \left(\overrightarrow{k l}+\varphi_{j}\right)+\vec{v}_{j} \sin \left(\overrightarrow{k l}+\varphi_{j}\right)
$$

et dans l'espace réciproque

$$
\vec{m}_{j}^{k}=\left(\frac{\vec{u}_{i}}{2}-i \frac{\vec{v}_{j}}{2}\right) e^{p_{j}}
$$

Nous avons donc deux vecteurs de base

$$
\begin{aligned}
& \frac{\vec{u}_{j}}{2} e^{i \varphi_{j}} \\
& -i \frac{\vec{v}_{j}}{2} e^{i \varphi_{j}}
\end{aligned}
$$

$m_{j}^{-k}$ étant associé à $m_{j}^{k}$ afin que $\vec{m}_{l j}$ soit réel.

Le facteur de structure magnétique est

$$
\vec{F}_{M}(\vec{Q})=p \sum_{j} f_{j}(\vec{Q})\left\lfloor\frac{\vec{u}_{j}}{2}-i \frac{\vec{v}_{j}}{2}\right\rfloor e^{i \varphi_{j}} e^{i \vec{Q} \vec{Q}_{j} e^{-\psi_{j}}}
$$

Du point de vue de la symétrie, cet objet se transforme comme une représentation irréductible à 2 dimensions qui peut être générée par des axes de symétrie 3,4 , ou 6 . Une telle configuration peut rester stable à basse température. 


\subsection{3- Les structures commensurables}

Dans la zone de Brillouin, les points de symétrie représentent des extrema pour le Hamiltonien $\mathrm{H}(\mathrm{k})$.

$$
\vec{k}=0,0,0
$$

correspond forcément à une structure ferromagnétique si on a un seul atome magnétique par maille. Elle peut être ferrimagnétique ou antiferromagnétique s'il y a plusieurs atomes par maille.

On a

$$
\vec{m}_{l j}=\vec{m}_{j}^{k}=\vec{m}_{0}
$$

Le coefficient de Fourier est égal au moment.

Si $\vec{k}$ est en un point de symétrie à la surface de la zone de Brillouin :

$$
\vec{m}_{l j}=(-1)^{\frac{\vec{k}}{n}} m_{j}^{k}
$$

on a une alternance de plans parallèles et antiparallèles.

Table 7 : classification des structures commensurables possibles

\begin{tabular}{|l|l|l|l|}
\hline & type I & type II & type III \\
\hline cubique P & $001 / 2$ & $1 / 21 / 20$ & $1 / 21 / 21 / 2$ \\
\hline cubique I & 001 & $1 / 21 / 20$ & \\
\hline cubique F & 001 & $1 / 21 / 21 / 2$ & \\
\hline
\end{tabular}

Pour une structure commensurable on a pour le facteur de structure

$$
\vec{F}_{M}(\vec{Q})=p \sum_{j} f_{j}(\vec{Q}) \vec{m}_{j} e^{i \bar{Q} \vec{r}_{j}} e^{-w_{j}}
$$

Le coefficient de Fourier est réel. On peut voir une structure commensurable comme une discontinuité pour une structure modulée.

\subsection{4-Structures mono-k et multi-k}

Par souci de simplicité, nous ne considérerons que des structures avec un seul atome par maille et que $\vec{m}^{k} / / \vec{k}$.

On a

$$
\vec{m}_{l}=\sum_{k} \vec{m}^{k} e^{-\bar{k} t}
$$

$\vec{k} /-\vec{k}$ sont déjà automatiquement associés.

De plus on supposera qu'il y a plusieurs vecteurs $\vec{k}$ équivalents : $\vec{k}_{1}, \vec{k}_{2}, \vec{k}_{3}$ 
Table 8 : Les modes magnétiques et l'Hamiltonien associé

\begin{tabular}{|l|l|l|}
\hline $\begin{array}{l}\text { ler } \\
\text { domaine }\end{array}$ & $\vec{m}_{l}=\vec{m}^{k_{1}} e^{-i \vec{k}_{l} I}(3.30)$ & $H_{1}=-J(k) \vec{m}^{k_{1}} \vec{m}^{-k_{1}}$ \\
\hline $\begin{array}{l}\text { 2ème } \\
\text { domaine }\end{array}$ & $\vec{m}_{l}=\vec{m}^{k_{2}} e^{-i \vec{k}_{2} l}(3.32)$ & $H_{2}=-J(k) \vec{m}^{k_{2}} \vec{m}^{-k_{2}}$ \\
\hline multi-k & $\begin{array}{c}\vec{m}_{l}=\vec{M}^{k_{1}} e^{-i \vec{l}_{1} I}+\vec{M}^{k_{2}} e^{-\vec{k}_{2} \vec{I}} \\
(3.34)\end{array}$ & $\begin{array}{c}H=-J(k)\left[\vec{M}^{k_{1}} \vec{M}^{-k_{1}}+\vec{M}^{k_{2}} \vec{M}^{-k_{2}}\right] \\
(3.35)\end{array}$ \\
\hline
\end{tabular}

si $M^{k_{1}}=\frac{1}{\sqrt{2}} m^{k_{1}}$ (double $\left.\mathrm{k}\right) ; M^{k_{1}}=\frac{1}{\sqrt{3}} m^{k_{1}}$ (triple k)

Soit le Hamiltonien magnétique suivant :

$$
\begin{aligned}
& H=-J \sum_{k_{i}} m^{k_{i}} m^{-k_{i}}+A_{4} \sum_{k_{i}}\left(m^{k_{i}}\right)^{4}+A_{4}^{\prime} \sum_{k_{i} k_{i^{\prime}}}\left(m^{k_{i}}\right)^{2}\left(m^{k_{i^{\prime}}}\right)^{2} \\
& +A_{6} \sum_{k_{i}}\left(m^{k_{i}}\right)^{6}+A_{6}^{\prime} \sum_{k_{i} k_{k^{\prime}}}\left(m^{k_{i}}\right)^{4}\left(m^{k_{i^{\prime}}}\right)^{2}+A_{6}^{\prime \prime} \sum \cdots
\end{aligned}
$$

On montre que pour un Hamiltonien limité à l'ordre 2, il n'y a pas de différence d'énergie entre une structure mono- $\mathrm{k}$ (avec domaines) et une structure multi-k

au 4ème ordre : la structure peut passer de mono-k à triple-k

au 6ème ordre : la structure peut passer de mono-k à double $k$ ou triple $k$ (terres rares)

\subsection{5- Les structures Multi-k}

Dans le cas général

$$
\vec{m}_{l}=\vec{m}^{k_{1}} e^{-i \overrightarrow{k_{1}} \vec{l}}+\vec{m}^{k_{2}} e^{-i \overrightarrow{x_{2}} \vec{l}}
$$

mais $\vec{m}_{l}$ est réel, pour tout $\vec{k}_{1} /-\vec{k}_{1}$ et pour tout $\vec{k}_{2} /-\vec{k}_{2}$

$$
\vec{m}_{l}=\vec{m}^{k_{1}} \cos \left(\vec{k}_{1} \vec{l}+\varphi_{1}\right)+\vec{m}^{k_{z}} \cos \left(\overrightarrow{k_{2}} \vec{l}+\varphi_{2}\right)
$$

Si nous faisons l'hypothèse que $\vec{m}^{k} / / \vec{k}$

nous avons les structures décrites ci-dessous. 


\subsubsection{Structure mono-k}

Table 9: Graphiques pour structures mono-k et sections efficaces

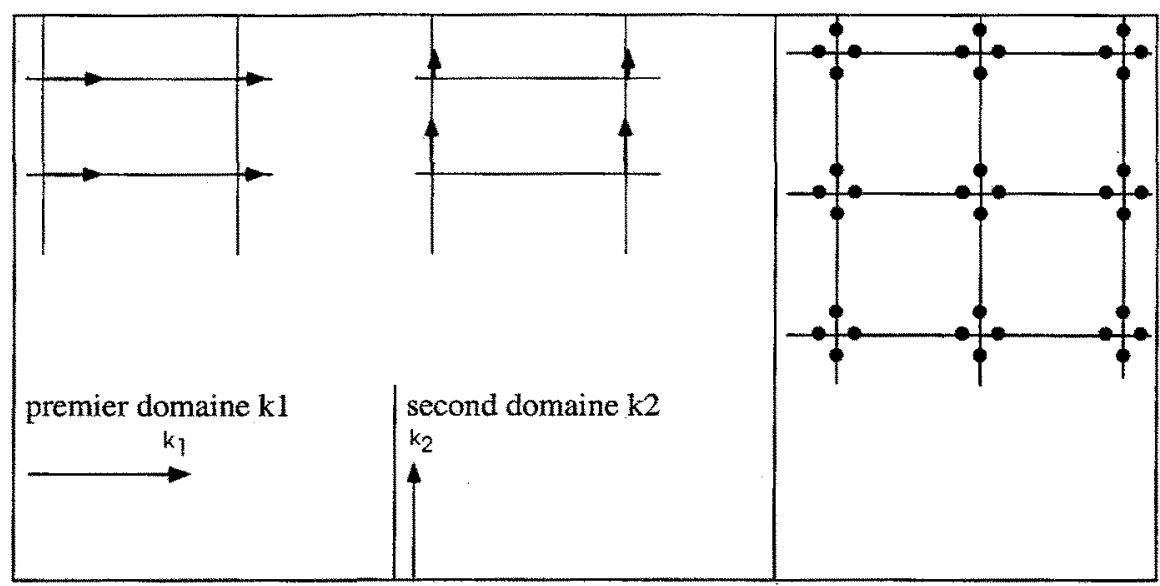

\subsubsection{Structure double $k$}

Table 10: Graphiques pour les structures double-k et les sections efficaces. Note: Attention, pour la structure mono-k, on obtient la même image dans l'espace réciproque et les intensités deviennent égales dès que l'on a une population égale des deux domaines.

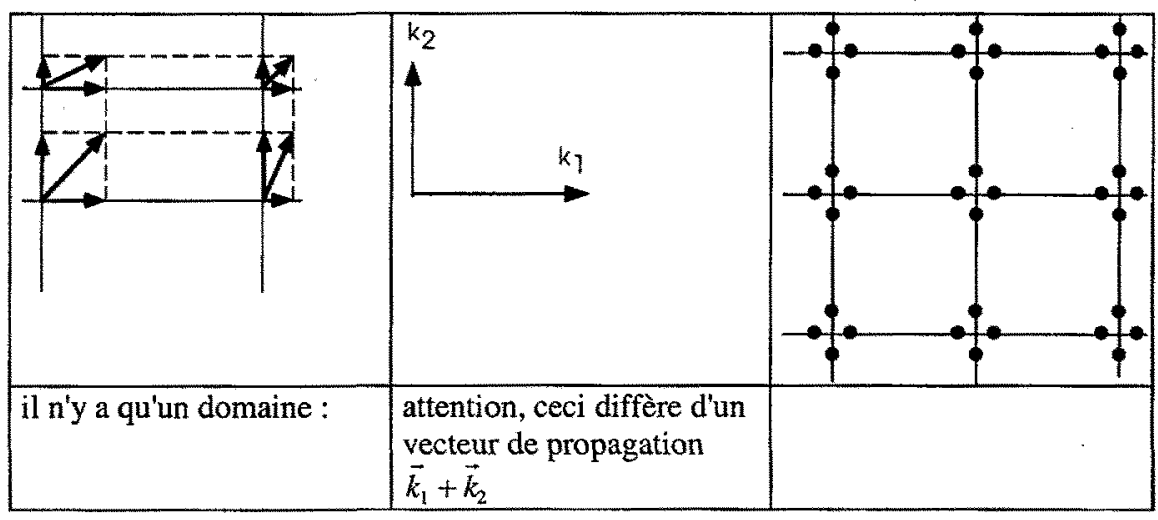




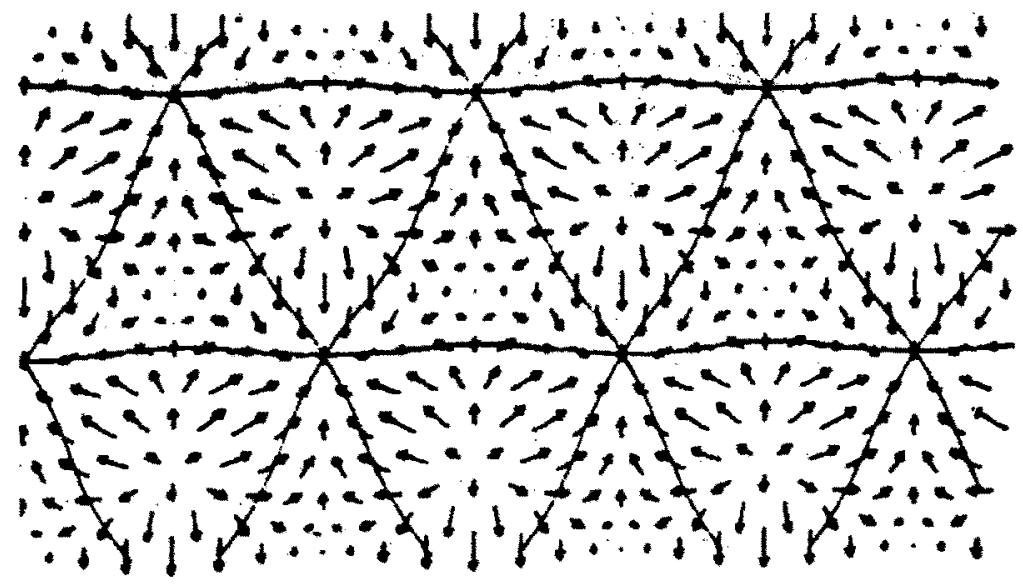

Fig. 19 : Exemple d'une structure triple $k$ :

\subsection{Comment enlever les ambiguités pour les structures multi-k}

3.2.1- Application d'une contrainte sur les multi-k

$$
\text { Single } \vec{k} \quad \text { Double }-\vec{k} \quad \text { Triple }-\vec{k} \quad \text { Four }-\vec{k}
$$
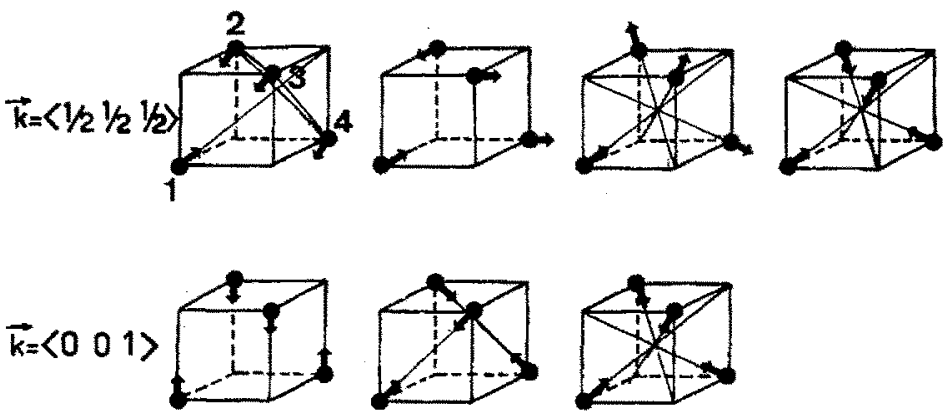

Fig. 20: structures multi-k

Exemple des structures multi- $\mathrm{k}$ les plus symétriques associées avec le vecteur de propagation $\mathrm{k}=$ $<1 / 2,1 / 2,1 / 2>$ et $k=<0,0,1>$ pour le réseau cubique à face centrées. Les ions $1,2,3,4$ sont au centre des faces du cube.

On voit sur l'exemple suivant que l'évolution des intensités des réflexions lorsqu'on applique une perturbation extérieure est différente.

UAs: L'application d'une contrainte uniaxiale déforme le réseau et favorise le domaine $\mathrm{k}$ pour lequel la modulation se propage parallèlement. On en déduit que l'ordre est mono-k. On peut penser qu'il l'était avant la contrainte.

USb: La même contrainte uniaxiale déforme le réseau, mais ne prođuit aucun changement dans les intensités. La structure reste triple $\mathrm{k}$ avec un seul domaine. 


\section{4- Diffusion des neutron polarisés}

\subsection{Théorie : Les cartes de densité magnétique atomiques et la dépendance de spin de la section efficace de diffusion des neutrons}

Soit $F_{N}(\vec{Q})[\mathrm{cm}]$ le facteur de structure nucléaire et $\vec{F}_{M}(\vec{Q})\left[\mu_{B}\right]$ le vecteur des facteurs de structure magnétique, $\vec{Q}$ étant le vecteur de diffusion. Dans un cristal magnétique, le moment magnétique du neutron interagit avec le champ d'induction magnétique périodique des électrons non-appariés résultant dans une amplitude de diffusion magnétique. A cause de la nature dipolaire de ce couplage seule la composante $\vec{F}_{M_{I}}(\vec{Q})$ qui est perpendiculaire à $\vec{Q}$ est effective.

$$
\vec{F}_{M L}(Q)=\hat{Q} \wedge\left(\vec{F}_{M}(\hat{Q}) \wedge \hat{Q}\right)
$$

On peut montrer que l'opérateur représentant l'amplitude de diffusion du neutron s'écrit dans l'espace de spin du neutron :

$$
a_{M}(\vec{Q})=p \vec{F}_{M \perp}(\vec{Q}) \cdot \vec{\sigma}
$$

L'opérateur de spin $\vec{\sigma}$ est un vecteur qui a 3 composantes. Chaque composante s'écrit dans l'espace de Hilbert à 2 dimensions du spin $1 / 2$ à l'aide des matrices de Pauli et

$$
p=0.269510^{-12}\left[\mathrm{~cm} / \mu_{B}\right]
$$

Est la longueur de diffusion associée à un magnéton de Bohr.

Il est très utile de définir le vecteur d'interaction magnétique :

$$
\vec{M}(\vec{Q})=p \vec{F}_{M \perp}(\vec{Q})
$$

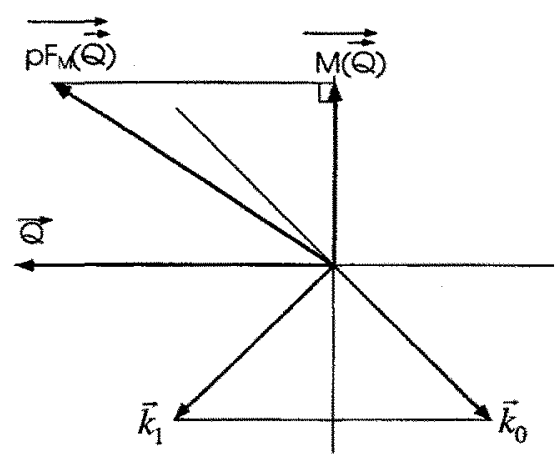

Figure 21 : Le vecteur d'interaction magnétique du neutron 
La section efficace dépendant du spin s'écrit :

$$
\begin{gathered}
\frac{\partial \sigma}{\partial \omega}=N N^{*}+\vec{P}\left(\vec{M} N^{*}+\vec{M}^{*} N\right)+\vec{M} \cdot \vec{M}^{*}+i \vec{P}_{i}\left(\vec{M}^{*} \wedge \vec{M}\right) \\
\text { avec } N=F_{N}(\vec{Q}) \text { et } \vec{M}=\vec{M}(\vec{Q})
\end{gathered}
$$

Pour simplifier nous allons supposer que le cristal est ferromagnétique ou paramagnétique avec un champ vertical appliqué et que nous sommes en train de mesurer des réflexions dans le plan horizontal. Nous faisons aussi l'hypothèse que la polarisation est \pm 1 , c'est-à-dire parallèle $(+)$ ou opposée (-) au champ magnétique appliqué. Alors :

$$
\begin{gathered}
\frac{\partial \sigma}{\partial \partial}=N N^{*}+M N^{*}+M^{*} N+M M^{*} \\
\text { avec } M=p\left|\vec{F}_{M}(\vec{Q})\right|=p F_{M}
\end{gathered}
$$

Si de plus le cristal a un centre de symétrie alors $N=N^{*}, M=M^{*}$ et l'on aboutit à l'expression suivante très simple pour le rapport des 2 sections efficaces généralement appelé rapport de flipping

$$
R=\frac{\sigma^{+}}{\sigma^{-}}=\left(\frac{N+M}{N-M}\right)^{2}
$$

A partir des valeurs expérimentales de $R(\vec{Q})$, les équations du 2ème degré précédentes ont deux solutions pour le rapport $M / N$. Le facteur de structure nucléaire ayant été mesuré par d'autres techniques les valeurs de $N$ sont connues et il est normalement facile de choisir la bonne valeur de $M$.

Grâce à l'existence des termes d'interférence magnetique-nucléaire, ces mesures nous fournissent des informations très sensibles sur l'amplitude des termes de diffusion magnétiques.

\subsection{Instrumentation : Exemple de diffractomètre à monocristaux équipé pour les mesures de neutrons polarisés sous haut champ magnétique : $D 3$ à I'ILL}

D3 est un diffractomètre à monocristaux recevant un faisceau incident de neutrons polarisés. En pratique l'instrument est positionné sur un pic de Bragg d'une structure cristalline préalablement connue. Simplement en renversant la polarisation du faisceau incident, D3 effectue une mesure très sensible du terme d'interférence nucléaire-magnétique qui se trouve dans la section efficace. Le petit monocristal échantillon est aimanté dans un champ intense allant jusqu'à 10 Tesla[6]. 


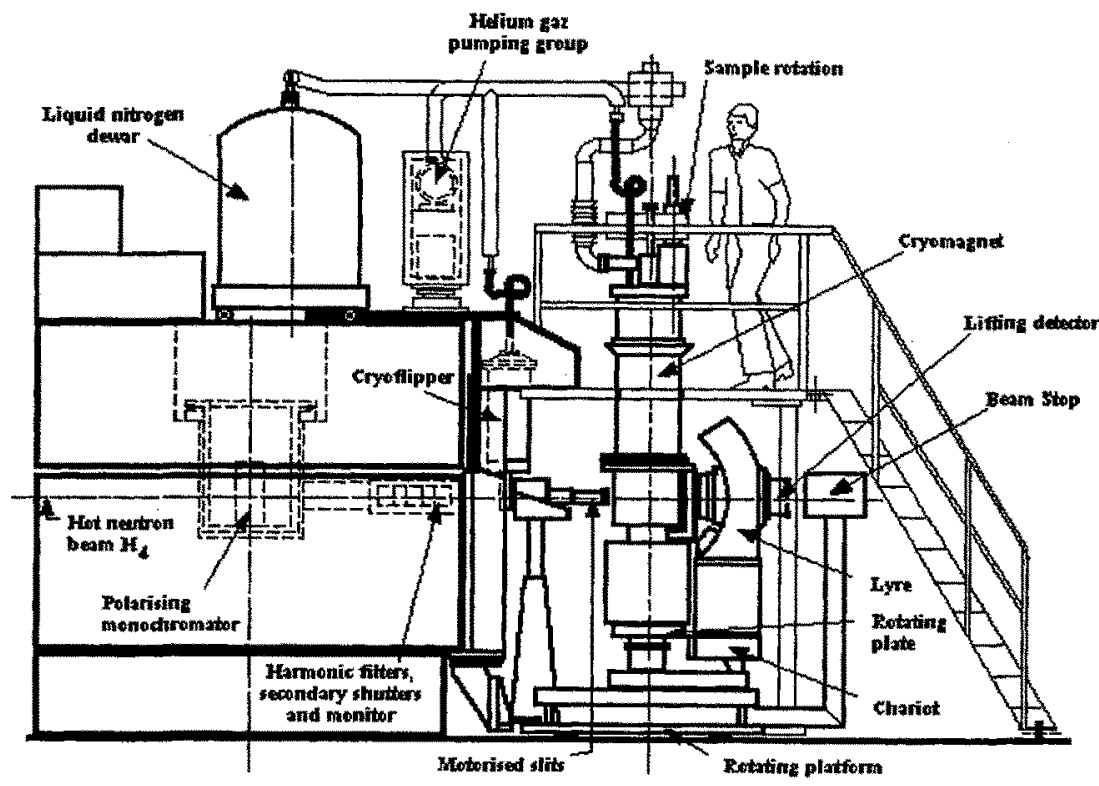

Fig 22: Le diffractomètre à neutrons polarisés D3 (http://www.ill.fr/YellowBook/D3/)

(a) Le faisceau de neutrons chaud. Depuis qu'il est installé sur le doigt de gant $\mathbf{H} 4 \mathrm{du}$ réacteur à haut flux de l'ILL, D3 exploitait les neutrons thermalisés par la source chaude de l'TLL avec des longueurs d'onde courtes allant jusqu'à $0.4 \AA$. La source chaude étant présentement en cours de réfection, on doit se limiter à $0.7 \mathrm{~A}$ et il faudra attendre 2001 pour un retour à la normale.

(b) Le spectromètre primaire polarisant. D3 dispose de deux cassettes monochromateur de CoFe et de $\mathrm{Cu}_{2} \mathrm{MnAl}$ contenant chacune un puissant aimant permanent apte à saturer complètement le cristal polariseur. Elles peuvent être facilement échangées. Le changement de longueur d'onde est automatique avec l'introduction d'un filtre métallique résonant pour diminuer la contamination harmonique. Ces changements de longueur d'onde sont particulièrement utiles quand le phénomène d'extinction affecte les mesures. Les calibrations sont normalement disponibles de 0.42 to $0.84 \AA$. Le flux de neutron le plus élevé est de $0.510^{7} \mathrm{~cm}^{-2} \mathrm{~s}^{-1}$. La polarisation du faisceau est élevée et dépend un peu de la longueur d'onde, du monochromateur utilisé et de la collimation en pile. Au moins égale à $90 \%$ elle peut atteindre $99 \%$ au plus haut. Le renversement de la polarisation (flipping) est effectué avec un cryoflipper insensible à la longueur d'onde et aux champs de fuite du cryoaimant. 
(c) Le diffractomètre "normal beam" à détecteur inclinable automatique.

L'axe secondaire peut se déplacer sur un sol de marbre à l'aide de coussins d'air "ILL-Tanzboden". Il est attaché mécaniquement à la sortie du monochromateur (Fig. 22). La géométrie "Normalbeam" combinée avec le détecteur inclinable permet d'explorer l'espace réciproque dans les 3 dimensions tout en gardant le cryostat vertical. De plus, le bras détecteur et la table échantillon étant essentiellement amagnétiques les cryoaimants à fort champ peuvent être utilisés dans de bonnes conditions mécaniques. Le détecteur s'incline vers le bas afin de libérer l'espace au-dessus du plan horizontal pour les gros cryostats. Un système de demi-volet pneumatiques est inséré devant le mono détecteur de $5 \mathrm{~cm}$ de diamètre afin de faciliter la détermination de la matrice d'orientation du cristal échantillon.

(d) Haut Champ et basse température sur l'échantillon. Presque toutes les mesures effectuées sur D3b nécessitent que l'échantillon soit maintenu à basse température et dans un champ magnétique intense. Pour cette raison, un cryoaimant à champ vertical de 10 Tesla est attitré à l'instrument. Il permet d'incliner le détecteur jusquà $-25^{\circ}$ vers le bas. Un contrôleur de température et une alimentation électrique pour le champ sont interfacés avec l'ordinateur de commande qui peut régler ces variables dans l'intervalle (1.0-10.0 Tesla, 1.5-300.0 Kelvin). Un sas étanche attaché au sommet du cryostat permet d'insérer des échantillons sensibles à l'air ou dangereux sans contact avec l'atmosphère.

(e) Acquisition de données. L'instrument est largement automatisé. Positionnement, comptage, stockage et transfert de données sont sous le contrôle d'un calculateur dédié. Le système d'acquisition comprend de nombreuses commandes simples (telle que "*SMF 9.6" pour établir le champ magnétique, "*WAV $.843^{*}$ pour choisir la longueur d'onde, suivi de "*SBH Er" pour introduire le filtre d'Erbium approprié) et des commandes plus sophistiquées pour collecter les données. Des fichiers d'input contenant de telles commandes peuvent être préparés. Les fichiers de sortie produits par les mesures sont stockés sur un serveur de fichiers. Ils peuvent être facilement analysés par un puissant système de programmes basés sur la bibliothèque CCSL [7]. Ceci permet une réduction rapide des données, un rassemblement des mesures équivalentes collectées dans les différents séquences d'acquisition pour faire des moyennes. Les facteurs de structure magnétiques ainsi obtenus sont alors transformés de Fourier pour une visualisation directe des densités magnétiques atomiques avant d'être utilisé pour affiner des modèles d'électrons magnétiques.

\subsection{Détermination de densités de spin Ferro, Ferri et Paramagnétique}

Les différentes façons d'exploiter ces coefficients de Fourier $M(k)$ ne serons pas développées ici car une discussion détaillée est donnée par J. Schweizer dans son cours "Form Factors and Magnetization densities" [8] 


\section{5- La polarimétrie neutronique sphérique : Analyse de polarisation du faisceau diffusé}

\subsection{Principe de la polarimétrie}

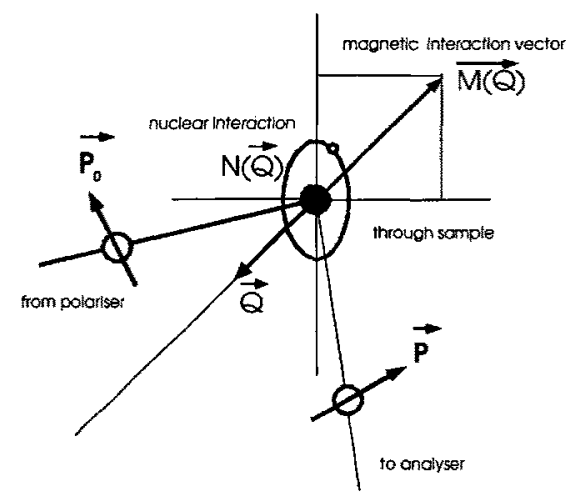

Fig. 23 : Polarimétrie Neutronique : Le faisceau incident est diffusé (ou diffracté) à un vecteur de diffusion $Q$. On oriente le vecteur polarisation de spin du faisceau incident dans une direction pertinente. La manière dont il est rendu nous renseigne sur la composante de Fourier des potentiels nucléaire et magnétique rencontrés dans l'échantillon.

\subsection{Théorie pour la polarimétrie neutronique élastique}

Dès 1963, la théorie complète de la diffusion neutronique élastique incluant le vecteur polarisation de spin, était publiée par M. Blume[9].

Deux équations étaient utilisées, la première décrivant la section efficace de diffusion et la seconde la polarisation finale en fonction de la polarisation initiale. Les deux équations contiennent les 2 interactions principales, nucléaire et magnétique, décrites dans la section précédente. En utilisant les mêmes notations que dans 2.2 la formule générale pour le vecteur de polarisation $\vec{P}$ des neutrons diffractés dans un pic de Bragg en partant d'une polarisation incidente $\vec{P}_{0}$ devient:

$$
\begin{aligned}
\sigma_{t}= & N N^{*}+\vec{P}_{0}\left(\vec{M} N^{*}+\vec{M}^{*} N\right)+\vec{M} \cdot \vec{M}^{*}+i \vec{P}_{0}\left(\vec{M}^{*} \times \vec{M}\right) \\
\vec{P} \sigma_{t}= & \vec{P}_{0} N N^{*}+\vec{M} N^{*}+\vec{M}^{*} N-i\left(\vec{P}_{0} \times \vec{M} N^{*}-\vec{P}_{0} \times \vec{M}^{*} N\right) \\
& +\vec{M}\left(\vec{P}_{0} \cdot \vec{M}^{*}\right)+\vec{M}^{*}\left(\vec{P}_{0} \cdot \vec{M}\right)-\vec{P}_{0}\left(\vec{M} \cdot \vec{M}^{*}\right)-i\left(\vec{M}^{*} \times \vec{M}\right)
\end{aligned}
$$

Ces 2 équations clefs peuvent être avantageusement découpées en 4 parties, chacune avec une origine physique bien identifiée que nous présentons alors dans la table suivante : 
La section efficace s'écrit :

$$
\sigma_{i}=\left(N N^{*}\right)+\left(\vec{M} \cdot \vec{M}^{*}\right)+\left(\vec{P}_{0} \cdot\left(\vec{M}^{*} \times \vec{M}\right)\right)+\left(\vec{P}_{0} \cdot\left(\vec{M} N^{*}+\vec{M}^{*} N\right)\right)
$$

et la polarisation finale:

$$
\begin{aligned}
\vec{P} \sigma_{t}=\left(\vec{P}_{0} N N^{*}\right) & +\left(-\vec{P}_{0}\left(\vec{M} \cdot \vec{M}^{*}\right)+\vec{M}\left(\vec{P}_{0} \cdot \vec{M}^{*}\right)+\vec{M}^{*}\left(\vec{P}_{0} \cdot \vec{M}\right)\right)+\left(-i\left(\ddot{M}^{*} \times \ddot{M}\right)\right) \\
& +\left(N \vec{M}^{*}+N^{*} \vec{M}+i\left(N \vec{M}^{*}-N^{*} \vec{M}\right) \times \vec{P}_{0}\right)
\end{aligned}
$$

\begin{tabular}{|c|c|c|}
\hline \multicolumn{3}{|c|}{$\begin{array}{l}\text { Equations clefs pour la polarimétrie neutronique Nucléa } \\
\qquad \sigma_{t}=\sigma_{n}+\sigma_{m}+\sigma_{c}+\sigma_{i} \\
\qquad \vec{P} \sigma_{t}=(P \vec{P})_{n}+(P \sigma)_{m}+(P \vec{\sigma})_{c}+(\overrightarrow{P \sigma})_{i}\end{array}$} \\
\hline (indexe) /mode & Contribution élastique & Commentaire \\
\hline nucléaire & $\begin{array}{l}\sigma_{n}=N N^{*} \\
(\overrightarrow{P \sigma})_{n}=\vec{P}_{0} \sigma_{n}\end{array}$ & $\begin{array}{l}\text { L'intensité varie comme le carré du } \\
\text { module du facteur de structure } \\
\text { nucléaire } \\
\text { La polarisation incidente est conservée }\end{array}$ \\
\hline $\begin{array}{r}(\mathrm{m}) \\
\text { magnétique } \\
\text { partie normale }\end{array}$ & $\begin{array}{l}\sigma_{m}=\vec{M} \cdot \vec{M}^{*} \\
(\overrightarrow{P \sigma})_{m}=-P_{0} \sigma_{m}\left\{\hat{P}_{o}-2\left(\hat{P}_{0} \cdot \hat{M}\right) \hat{M}\right\}\end{array}$ & $\begin{array}{l}\text { L'intensité "normale" varie comme le } \\
\text { carré du module du Vecteur } \\
\text { d'Interaction Magnétique (MIV) } \\
\text { La polarisation incidente est renversée } \\
\text { sauf pour la composante selon le MIV }\end{array}$ \\
\hline $\begin{array}{r}(\mathrm{c}) \\
\text { magnétique } \\
\text { partie chirale }\end{array}$ & $\begin{array}{l}\sigma_{c}=i\left(\vec{M}^{*} \times \vec{M}\right) \vec{P}_{0} \\
(P \vec{\sigma})_{c}=-i\left(\ddot{M}^{*} \times \vec{M}\right)\end{array}$ & $\begin{array}{l}\text { L'intensité "chirale" dépend de la } \\
\text { polarisation initiale } \\
\vec{M} \times \vec{M}^{*} \neq 0 \text {, la chiralité, crée de la } \\
\text { polarisation dans la direction du } \\
\text { vecteur de diffusion }\end{array}$ \\
\hline $\begin{array}{r}\text { (i) . interférence } \\
\text { nucleaire- } \\
\text { magnétique }\end{array}$ & $\begin{array}{l}\sigma_{i}=\left(N \vec{M}^{*}+N \vec{M}\right) \cdot \vec{P}_{0} \\
(P \vec{\sigma})_{i}=N \vec{M}^{*}+N^{*} \vec{M} \\
\quad+i\left[\left(N \vec{M}^{*}-N^{*} \vec{M}\right) \times \vec{P}_{0}\right]\end{array}$ & $\begin{array}{l}\text { L'intensité dépend de la polarisation } \\
\text { incidente lorsque N\&M sont en phase } \\
\text { De la polarisation est créé selon } \vec{M} \\
\text { quand N\&M sont en phase. } \\
\text { La polarisation incidente est tournée } \\
\text { sur un cône autour de } \vec{M} \text { quand N\&M } \\
\text { sont en quadrature. }\end{array}$ \\
\hline
\end{tabular}

Table 11 : Les 4 contributions principales dans la diffusion polarimétrique des neutrons : 
En un point $\vec{Q}$ de l'espace réciproque, l'opérateur amplitude de diffusion est

$$
F(\vec{Q})=N(\vec{Q})+\vec{M}(\vec{Q}) \cdot \vec{\sigma}
$$

$N(\vec{Q})=-n^{-\frac{1}{2}} \sum_{n} b_{n} e^{i \vec{Q} \cdot \vec{k}_{n}}$ est le facteur de structure nucléaire

$\vec{M}(\vec{Q})=p \vec{F}_{M \perp}(\vec{Q})$ est le vecteur d'interaction magnétique (MIV)

$$
\begin{gathered}
p=0.269510^{-12}\left[\mathrm{~cm} / \mu_{B}\right] \\
\left.\vec{F}_{M 1}(\vec{Q})=p \sum_{m} f_{m}(\vec{Q}) \vec{n}_{m}-\left(\hat{Q} \cdot \vec{m}_{m}\right) \hat{Q}\right]^{i \vec{Q} \cdot k_{m}} e^{-W_{m}}
\end{gathered}
$$

Les 3 composantes de $\vec{\sigma}$ sont les matrices de Pauli (vecteur opérateur), $\vec{r}_{m}$ est la position de l'atome magnétique $\mathrm{m}, \vec{m}_{m}$ le moment magnétique localisé en magnétons de Bohr $\mu_{B}$, et $f_{m}(\vec{Q})$ est le facteur de forme magnétique normalisé à 1 en $\mathrm{Q}=0$.

5.2.1 Comment les 3 composantes de la polarisation finale peuvent détecter la configuration magnétique absolue

En faisant l'hypothèse que la partie imaginaire et la partie réelle du vecteur interaction magnétique complexe $\vec{M}$ sont parallèles (i.e. $\ddot{M}=M \cdot \hat{M}$ ), le terme chiral disparaît. Une représentation intéressante des directions de polarisation[10] est représentée dans un système de coordonnés où l'axe polaire est parallèle à $\vec{M}$

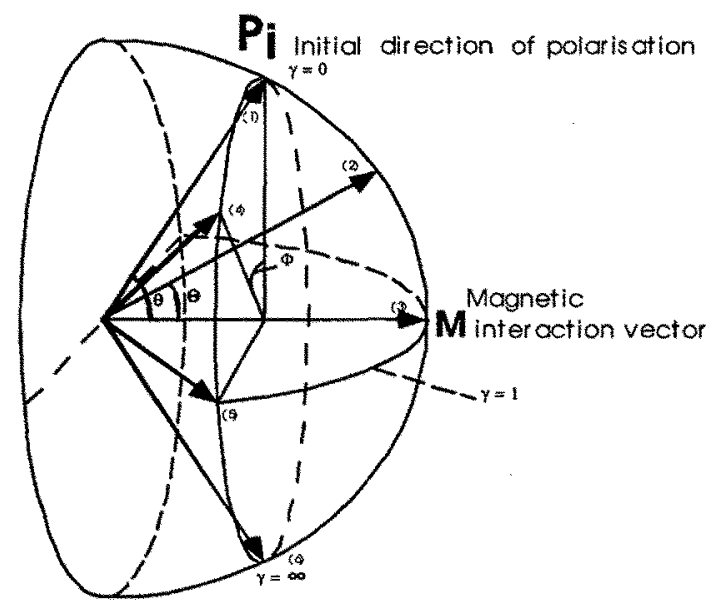

Fig 24 : Rotation du vecteur polarisation du neutron $\vec{P}$ relativement à la direction de $\vec{M} . \gamma_{\text {est un }}$ nombre qui exprime la longueur du vecteur interaction magnétique $\vec{M}$ en unitê de facteur de structure $N$. Les directions particulières (i) sont discutées dans le texte. 
Alors, en utilisant le jeu de coordonnées polaires ordinaires et en faisant l'hypothèse que $P_{i}=1$ :

$$
\begin{gathered}
\vec{P}_{i}\left[1, \theta_{i}, \varphi_{i}\right], \quad \vec{P}_{f}\left[1, \theta_{f}, \varphi_{f}\right], \quad \text { et } \delta \varphi=\varphi_{f}-\varphi_{i} \\
\delta \varphi=\cos ^{-1}\left\lfloor\frac{N N^{*}-M M^{*}}{\sqrt{\left(N N^{*}+M M^{*}\right)^{2}-\left(N M^{*}+N^{*} M\right)^{2}}}\right\rfloor \\
\cos \theta_{f}=\frac{\left(N N^{*}+M M^{*}\right) \cos \theta_{i}+N M^{*}+N^{*} M}{N N^{*}+M M^{*}+\left(N M^{*}+N^{*} M\right) \cos \theta_{i}}
\end{gathered}
$$

A ce niveau, il est utile d'introduire le rapport complexe

$$
\Gamma=\frac{M}{N}
$$

Quand $M$ et $N$ sont en phase, $\Gamma$ est un nombre réel. Quand ils sont en quadrature c'est un nombre imaginaire pur.

Nous pouvons faire les remarques suivantes: (les nombres entre parenthèse sont ceux de la Fig. 24) - $\theta$ et $\varphi$ sont complètement découplés quand $\Gamma$ est réel ou imaginaire pur.

-L'angle de "précession" $\delta \varphi$ dont tourne le plan $(\vec{M}, \vec{P})$ autour de $\vec{M}$ est indépendant de l'angle $\varphi$ initial.

De plus :

-Si $\Gamma$ est réel $\delta \varphi$ est nul. La polarisation reste dans le plan $(\vec{M}, \vec{P})$ initial. Elle ne précesse pas mais s'incline vers $\vec{M}$ quand $\Gamma$ augmente. En partant naturellement de la direction (1) (direction initiale) pour $\Gamma=0$ (nucléaire pur) elle passe en (2) quand $\Gamma$ augmente et devient colinéaire à $\pm \vec{M}$, (3), quand $\Gamma= \pm 1$. Pour $\Gamma= \pm \infty$ (réflexion magnétique pure) elle est retournée dans la direction opposée (6) (comme si elle avait precessé de $\pi$ autour de $\vec{M}$ ).

-Si $\Gamma$ est imaginaire pur $\theta_{i}=\theta_{f}$, la composante de polarisation parallèle à $\vec{M}$ reste inchangée. On peut dire que la polarisation "précesse" autour de $\vec{M}$ d'un angle $\delta \varphi$ sans changement de l'angle de nutation $\theta . \delta \varphi$ varie de $-\pi$ pour $\Gamma=-i \infty(6)$ à $\pi$ pour $\Gamma=i \infty(6)$ en passant par $\pi / 2$ (5) quand $|\Gamma|=1$. Le signe de $\delta \varphi$ est le même que celui de $\Gamma$, la polarisation tourne positivement autour de $\vec{M}$ quand $\Gamma$ est positif.

-On remarque la cohérence entre les 4 chemins différents qui mènent vers le cas magnétique pur $(\Gamma= \pm \infty ; \quad \Gamma= \pm i \infty)$. Tous se rendent vers le même point en passant par 4 chemins différents sur la sphère),

-Dans le cas général où $\Gamma$ est complexe la situation se complique : Les contributions "réelles" et "imaginaires" à la polarisation ne sont pas simplement additives à cause des termes venant des sections efficaces dépendant du spin. Néanmoins, la polarisation finale reste dans des quadrants distincts de la représentation sphérique, avec les frontières bien définies et les points spéciaux discutés plus haut. Ces contraintes géométriques apportent souvent des recettes approximatives pour la direction, la grandeur et la phase du vecteur interaction. L'analyse complète de la mesure [11] nécessite naturellement des calculs sur ordinateur [7] qui s'appuient sur le formalisme complet et qui prennent en compte la symétrie du problème (Les domaines différents donnent des Ms différents). Chaque $\mathrm{M}$ donne une polarisation finale différente. Les polarisations ne sont pas simplement additives car elles sont pondérées par les sections efficaces [10]. 


\subsubsection{Théorie spéciale pour la composante longitudinale de polarisation finale LPA}

Historiquement, le premier arrangement fait pour observer la polarisation finale diffusée en dehors du faisceau direct a été réalisé à Oak-Ridge. La direction de polarisation initiale $\vec{P}_{0}$ était fixée adiabatiquement à l'aide d'un champ de guidage relativement fort appliqué au niveau de l'échantillon. Cet axe particulier, pas forcément vertical était appelé $z$ et choisi comme axe de quantification pour décrire les états de spin $(t,-)$ du neutron (voir l'appendice). Dans un tel champ de guidage, la seule composante de la polarisation finale $\vec{P}$ qui est conservée pour être analysée ultérieurement est $P_{z}$, la "composante longitudinale". Moon Riste et Koehler [12] étant persuadés que l'on ne pouvait pas travailler sans un champ de guidage ont focalisé leur attention sur cette composante. Ils produisirent une théorie simple pour interpréter les 4 sections efficaces indexées par l'état de spin dans cette direction $z$ unique et qui sont devenues fameuses :

$$
\begin{gathered}
\sigma_{z z}^{++}=\left\langle b+p S_{\perp}^{z}\right\rangle^{2} \\
\sigma_{z z}^{-}=\left\langle b-p S_{\perp}^{z}\right\rangle^{2} \\
\sigma_{z z}^{+-}=\left\langle p \cdot\left(S_{\perp}^{x}+i S_{\perp}^{y}\right\rangle^{2}\right. \\
\sigma_{z z}^{++}=\left\langle p \cdot\left(S_{\perp}^{x}-i S_{\perp}^{y}\right\rangle^{2}\right.
\end{gathered}
$$

Nous avons ajouté en indice pour les sections partielles $\sigma$ les 2 axes de quantification zz (avant et après diffusion) qui etaient restés implicites dans la pensée de MRK et donc malheureusement absents dans leur notation. En utilisant le polarimètre en champ nul Cryopad nous avons maintenant accès aux 12 sections indépendantes $\left(\sigma_{z z}^{++}, \sigma_{z z}^{--}, \sigma_{z z}^{+-}, \sigma_{z z}^{+}, \sigma_{z x}^{++}, \sigma_{z x}^{--}, \sigma_{z x}^{+-}, \sigma_{z x}^{-+}, \sigma_{z y}^{++}, \sigma_{z y}^{-}, \sigma_{z y}^{+-}, \sigma_{z y}^{-+}\right)$qui sont exprimés de manière plus claire à l'aide du formalisme publié antérieurement par Blume et basé sur le concept universellement connu de vecteur de polarisation, c'est-à-dire sur les asymétries des sections efficaces (Il faut faire attention que pour décrire les résultats de SNP $z$ est toujours choisi vertical)

Cependant, et pour plus de 20 ans, LPA (malencontreusement appelée Polarisation Analysis par ses inventeurs) était la première technique disponible a $\vec{Q}$ fini. Les expérimentateurs se sont habitués au formalisme des sections efficaces partielles et ont oublié les hypothèses simplificatrices, jusqu'au point ou la méthode LPA fut appelée "Full Polarisation Analysis" par des promoteurs trop enthousiastes!

La mesure de tels processus Spin-Flipped SF $(+-;+)$ et Non-Spin-Flipped NSF $(++,-\cdots)$ dans la direction $z$ du champ de guidage étant la seule possible quand des études « sous champ " doivent être effectuées, il est devenu habituel d'utiliser cette théorie simplifiée qui est la seule présentée dans la plupart des livres.

Nous recommandons d'utiliser "Longitudinal Polarisation Analysis" (LPA) pour décrire cette technique et d'être plus prudent envers une interprétation trop optimiste des résultats : comme la technique LPA n'est pas capable, par définition, de détecter les composantes transversale de polarisation nous préférons utiliser la SNP dans toutes les études sur les antiferromagnétiques où l'application d'un champ magnétique n'est pas nécessaire.

Par exemple il est souvent affirmé que la LPA peut séparer les termes nucléaires (b) des termes magnétiques (p) dans l'interaction : en mettant l'axe de quantification z (i.e. le champ de guidage) le long du vecteur de diffusion, on peut annuler géométriquement le terme magnétique sans spinflip p. $\mathbf{S}_{\perp}^{2}$. Ainsi tous les termes nucléaires deviennent non-spin-flip et les termes magnétiques spin-flip selon $z$. Cette méthode est vraie et facile mais peu sensible ! 
Sachant les problèmes de statistique qui sont presque toujours présents dans ces mesures qui nécessitent l'analyse de la polarisation finale, la méthode peut facilement manquer de détecter un petit changement de celle-ci, seul indicateur d'une corrélation nucléaire magnétique pourtant bien excitante. Nous verrons qu'une telle corrélation produit une rotation sensible de la polarisation et donc une composante transverse de polarisation. La mesure LPA ne prouve donc pas vraiment que ces termes d'interférence nucléaire-magnétique sont nul car elle est peu sensible à cela. C'est seulement lorsque les 2 amplitudes deviennent comparables que la polarisation est largement tournée et que la composante longitudinale est vraiment réduite. Tant que les 2 composantes sont déséquilibrées (faible effet nucléaire dans un fort magnétisme ou l'inverse) elles ne peuvent être facilement détectés qu'à l'aide de 2 effets : la dépendance de la section efficace avec la polarisation initiale si les 2 amplitudes sont en phase, la composante transverse de polarisation si elles sont en quadrature de phase. C'est en cela que les techniques de neutron polarisés sont vraiment sensibles et méritent d'être vraiment poussées.

\subsection{Aspects expérimentaux}

\subsubsection{La technique de la chambre en champ nul}

Pendant longtemps, la théorie de l'analyse de polarisation généralisée [9] avait prédit que la mesure des trois composantes de la polarisation finale était intéressante, parfois la seule manière de décider entre deux configurations magnétiques [13]

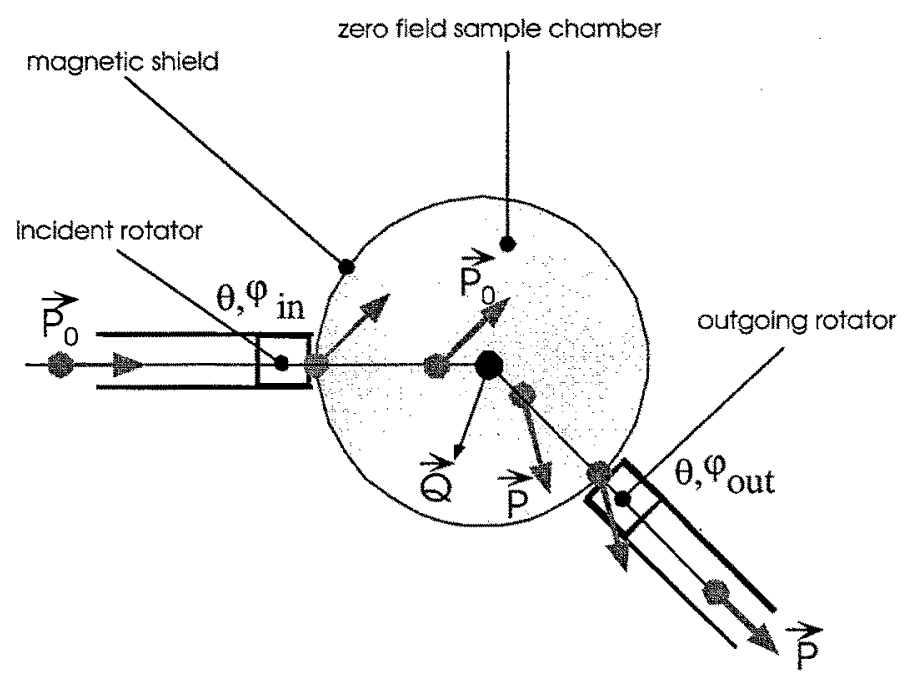

Fig. 25: Principe de la polarimétrie neutronique en champ nul

\subsubsection{Cryopad}

Une telle mesure n'est pas facile à cause de l'influence importante de tout champ magnétique sur la polarisation des neutrons. Elle requiert de raccorder 2 champs magnétiques de guidage dans des directions différentes avec une chambre de champ nul. Nous avons réussi à résoudre ce problème de 
manière radicale dans notre polarimètre neutronique Cryopad en construisant la chambre échantillon avec des écrans magnétiques supraconducteurs à effet Meissner.[3]

Cryopad est donc un outil qui permet d'effectuer le contrôle complet du vecteur polarisation ainsi qu'il apparait nécessaire pour exploiter au mieux la théorie présentée en section 5.2 [9]. Lorsqu'il est installé sur un faisceau monochromatique polarisé provenant d'un spectromètre primaire, Cryopad permet d'orienter le vecteur polarisation dans une direction appropriée avant qu'il rentre dans la chambre échantillon. Cette chambre est maintenue dans un état de vide magnétique (au maximum quelques $\mathrm{mG}$ ) afin de minimiser la précession résiduelle qui doit rester de l'ordre du degré pour satisfaire nos objectifs. De la sorte le changement de polarisation résulte uniquement du processus de diffusion dans l'échantillon dont l'aimantation macroscopique doit être nulle. Lorsque la polarisation finale sort de la chambre échantillon, Cryopad agit sur elle pour amener une après l'autre les 3 composantes dans la direction du champ de guidage final qui va les transporter jusqu'à l'analyseur. Plus de détails sur la conception de ce polarimètre et sur la technique de l'analyse de polarisation généralisée se trouvent dans [3], [11],[14]

(a) Le spectromètre hôte. Cryopad peut s'utiliser comme une option sur le spectromètre triple-axes IN20 qui dispose d'un cristal de Heusler polarisant sur le premier et le dernier axe. Il peut également s'installer sur le diffractomètre à neutron polarisés D3 en insérant un filtre de $3 \mathrm{He}$ polarisant devant le détecteur.

Une description à jour de IN20 et D3 se trouve sur les pages web de 1'ILL (www.ill.fr). Sur IN20, lors de nos mesures, les arrangements de cristaux de Heusler étaient à focalisation verticale (120mm de haut pour le monochromateur et $85 \mathrm{~mm}$ pour l'analyseur) en géométrie de Bragg symétrique avec le champ magnétique appliqué horizontalement dans le plan miroir et un gap de $75 \mathrm{~mm}$ ]. Les deux instruments vont être améliorés dans les années qui viennent dans le cadre du Millénium programme de l'ILL.

(b) Le système d'acquisition de données. Sur IN20, Cryopad est piloté par une version spéciale du software inélastique de l'ILL. Cela permet de mettre la polarisation incidente dans une direction arbitraire et de mesurer la polarisation finale dans n'importe quelle direction. Des courants appropriés sont automatiquement introduits dans les bobines en tenant compte de l'énergie différente dans les deux bras du spectromètre. Sur D3 Cryopad est conduit par une version spéciale du programme cristallographique d'acquisition de données qui intègrent des commandes spéciales pour la polarisation.

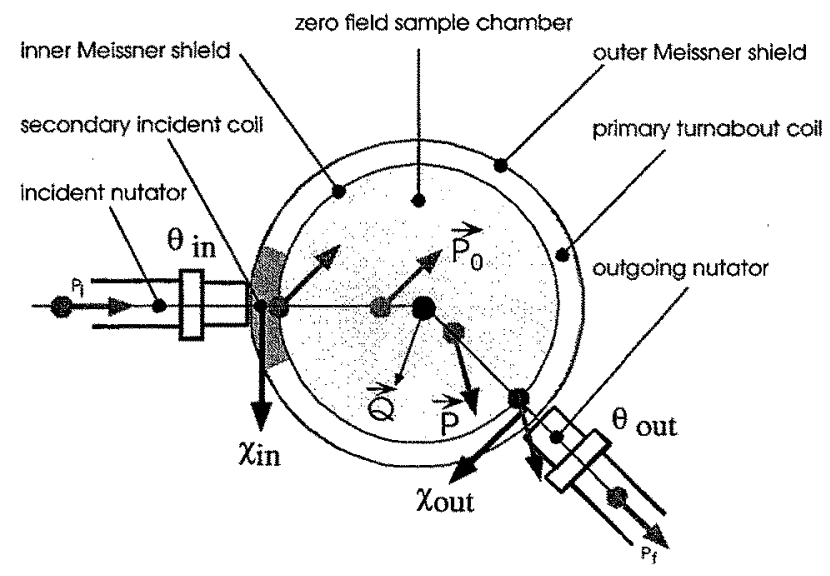

Fig 26 Vue Schématique des différentes régions magnétiques dans Cryopad 


\subsubsection{LPA dans un champ guide}

La première mesure systématique utilisant la méthode d'analyse de polarisation a été développée à Oak-Ridge par Moon Riste et Koehler [MRK] [12]. Utilisant un spectromètre 3 axes sur un faisceau thermique du HFIR, le contrôle de la direction de polarisation $\mathrm{z}$ au niveau de l'échantillon se faisait avec un champ magnétique de guidage.

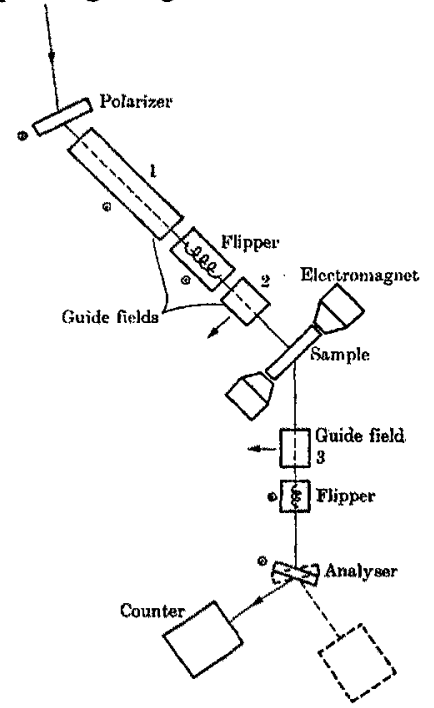

Fig. 27: Représentation schématique d'un spectromètre 3 axes équipé pour l'analyse de polarisation (d'après [12])

Du fait que seule la composante $\mathrm{z}$ de polarisation longitudinale (parallèle au champ et donc à la polarisation incidente) est mesurée, les autres composantes étant délibérément perdues au cours de la mesure (comme on le voit sur la figure suivante) nous l'appelons "Longitudinal Polarisation Analysis" LPA

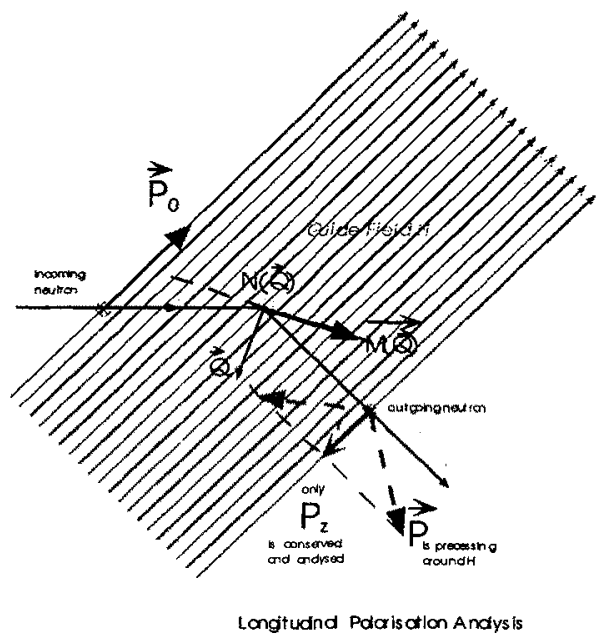

Fig. 28 : LPA : Effet d'un champ magnétique de guidage sur la polarisation diffusée. 


\section{6- La polarimétrie neutronique sphérique en action}

\section{1-Utilisation de la polarimétrie neutronique sphérique pour la détermination des structures magnétiques [16]}

La détermination des structures magnétiques est fondamentalement différente de la détermination de structures cristallographiques traditionnelles. Dans ces dernières, le but recherché est de déterminer les positions atomiques alors que pour des structures magnétiques, les sites des atomes magnétiques sont en principe déjà connus, mais la grandeur ainsi que la direction des moments magnétiques doivent être déterminés. De plus le facteur de structure magnétique diffère du facteur de structure conventionnel en étant un vecteur complexe, plutôt qu'une quantité scalaire complexe. Puisque dans une expérience classique de diffraction seule l'intensité des réflexions de Bragg peut être mesurée, le problème de la phase est aggravé dans le cas magnétique parce que 6 quantités, les amplitudes et les phases des 3 composantes des facteurs de structure, doivent être déduites d'une seule mesure d'intensité. En fait, à cause de la forme particulière de l'interaction dipolaire entre le neutron et l'induction magnétique dans le cristal, seules les composantes du facteur de structure magnétique perpendiculaires au vecteur de diffusion contribuent à l'intensité diffusée et c'est cette particularité qui permet éventuellement de déterminer la direction du moment. Cependant, dans les structures complexes en particulier celles qui sont non colinéaires, la seule mesure de l'intensité magnétique peut se révéler insuffisante pour déterminer la structure magnétique de manière unique. C'est dans ce contexte que la polarimétrie neutronique peut se révéler extrêmement utile, le changement de direction du vecteur de polarisation au terme du processus d'interaction étant assez directement lié à la direction du vecteur interaction magnétique (voir la table ci-dessous pour un résumé graphique).

6.1.1- Approche graphique de la SNP [10]

$$
\begin{gathered}
\sigma_{t}=\sigma_{n}+\sigma_{m}+\sigma_{c}+\sigma_{i} \\
\vec{P} \sigma_{t}=(P \vec{\sigma})_{n}+(P \vec{\sigma})_{m}+(P \vec{\sigma})_{c}+(P \vec{\sigma})_{i}
\end{gathered}
$$


Tableau 12 : Les 4 parties principales de la Diffusion polarimétrique élastique des neutrons (vue graphique). Remarquons que le cas $a=b$ pour la partie chirale magnétique (c) ne doit pas être considéré comme une restriction : ce qui reste de la composante la plus longue agit simplement comme un vecteur interaction magnétique normal (m).

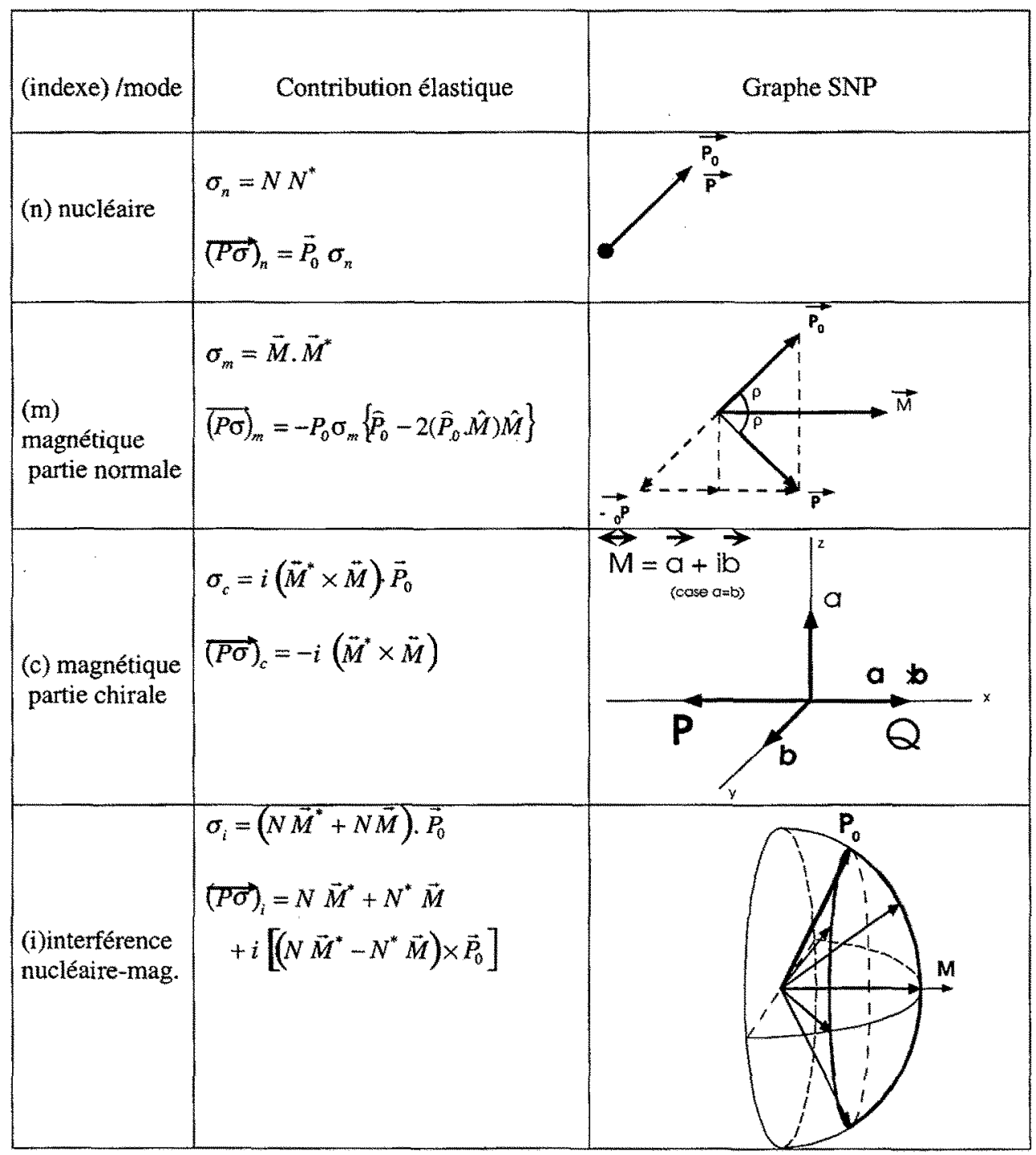




\subsection{2- Dépolarisation due aux domaines antiferromagnétiques}

Les équations qui relient le vecteur polarisation diffusée au vecteur incident ont été démontrées par Blume[9]. La polarisation $\vec{P}$ obtenue pour une réflexion de Bragg avec un vecteur de diffusion $\vec{Q}$ peut s'écrire en fonction du vecteur interaction magnétique $\vec{M}(\vec{Q})$ comme dans l'équation (5.2). Le module au carré du second membre de cette équation

$$
\begin{aligned}
\vec{P} \sigma_{i}= & \vec{P}_{0} N N^{*}+\vec{M}^{*}+\vec{M}^{*} N-i\left(\vec{P}_{0} \times \vec{M} N^{*}-\vec{P}_{0} \times \vec{M}^{*} N\right) \\
& +\vec{M}\left(\vec{P}_{0} \cdot \vec{M}^{*}\right)+\vec{M}^{*}\left(\vec{P}_{0} \cdot \vec{M}\right)-\vec{P}_{0}\left(\vec{M} \cdot \vec{M}^{*}\right)-i\left(\vec{M}^{*} \times \vec{M}\right)
\end{aligned}
$$

est toujours plus grand ou égal à $\left|\vec{P}_{0}\right|^{2} \sigma_{t}^{2}$ ce qui veut dire que l'amplitude de la polarisation est soit augmentée soit inchangée par diffusion sur un état propre du système, alors que sa direction sera changée. Une réelle dépolarisation du faisceau diffusé résulte d'un mélange d'états (plus d'un domaine magnétique). La possibilité de distinguer une vraie dépolarisation d'une rotation triviale du vecteur de polarisation hors de sa direction initiale est une des particularités qui rend la technique SNP plus puissante que l'analyse de polarisation longitudinale conventionnelle (LPA).

\subsection{3.-Classification des domaines}

Il est utile à ce point de rappeler les divers types de domaines magnétiques qui peuvent exister dans les structures antiferromagnétiques. Ils peuvent être classés de la manière suivante :

- Domaines de configuration (domaines $\mathrm{K}$ ),

- Domaines à $180^{\circ}$, (i.e. domaines Magnétoélectriques )

- Domaines d'orientation, (domaines $\mathbf{S}$ )

- Domaines de chiralité.(domaines LR)

Les types de domaines pouvant exister, et leur nombre, dépendent des symétries relatives entre la phase paramagnétique et la phase magnétique ordonnée. En général, si l'ordre du groupe paramagnétique est $\mathrm{p}$ et celui du groupe de symétrie magnétique est $\mathrm{m}$, le nombre de domaines magnétiques différents est $\mathrm{p} / \mathrm{m}$.

\subsubsection{1-Domaines de Configuration (domaines $\mathrm{K}$ )}

Comme nous l'avons dit dans la section 1 , des domaines de configuration naissent dès lors que le vecteur de propagation $\vec{k}$ décrivant la structure magnétique n'est pas transformé en lui même, ou en lui-même plus un vecteur du réseau réciproque, par tous les éléments de symétrie du groupe paramagnétique. Alors, laction de la symétrie paramagnétique sur $\vec{k}$ génère un jeu de vecteurs inéquivalents qui forment une étoile de $\vec{k}$. Chaque vecteur de létoile génère un domaine de configuration différent et chaque domaine de configuration donne naissance à un jeu séparé de réflexions magnétiques.

\subsubsection{2- Domaines à $180^{\circ}$}

Les domaines à 180 correspondent à des régions du cristal dans lesquelles toutes les directions des moments dans un domaine sont renversées par rapport aux moments correspondant dans l'autre domaine. Dans une structure à vecteur de propagation non nul de tels domaines ne peuvent pas être distingués sauf à partir des défauts associés aux parois de domaines. Une translation $\vec{\tau}$ telle que $\vec{\tau} \cdot \vec{k}=n$, transforme un domaine dans l'autre. Quand le vecteur de propagation est nul, la diffusion 
magnétique et nucléaire se retrouvent dans les mêmes réflexions et la différence de phase entre les deux amplitudes change de $\pi$ pour une paire de domaines à $180^{\circ}$. Deux types de structures antiferromagnétiques à $\vec{k}$ nul peuvent être distingués. Dans le premier, la diffusion magnétique et nucléaire diffèrent en phase de zéro ou $\pi$. Ce sont des structures centrosymétriques dans lesquelles les moments reliés par le centre de symétrie sont parallèles entre eux. Un exemple est $\mathrm{Fe}_{2} \mathrm{O}_{3}$, dont une projection illustrant les 2 domaines possibles est montrée dans la fig. 29. Pour de telles structures la section efficace

$$
\sigma_{i}=\sigma_{n}+\sigma_{m}+\sigma_{c}+\sigma_{i}
$$

dépend de la polarisation incidente à cause de la contribution des termes d'interférence nucléairemagnétique $\sigma_{i}=\left(N \vec{M}^{*}+N \vec{M}\right) \cdot \vec{P}_{0}$, eq. (5.13), puisque ces termes, linéaires en $\mathrm{M}$, ont des signes opposés pour les deux domaines. La polarisation du faisceau diffusé est tournée dans le plan de la polarisation incidente $\vec{P}_{0}$ et de $\vec{M}(\vec{Q})$ à cause des termes $\left(-\vec{P}_{0}\left(\vec{M} \cdot \vec{M}^{*}\right)+\vec{M}\left(\vec{P}_{0} \cdot \vec{M}^{*}\right)+\vec{M}^{*}\left(\vec{P}_{0} \cdot \vec{M}\right)\right)+\left(N \vec{M}^{*}+N^{*} \vec{M}\right)$ dans eq. (5.4)

Pour la deuxième classe de structures, l'amplitude de diffusion nucléaire et magnétique diffère en phase de $\pm \frac{\pi}{2}$, ceci se produisant automatiquement quand les moments reliés par un centre de symétrie sont antiparallèles. Dans ce cas, la section efficace ne dépend plus de la polarisation incidente car la quadrature de phase entre $\mathrm{N}$ et $\mathrm{M}$ annule le terme dépendant de la polarisation. Cependant, le deuxième terme dans eq $(5.14)$, qui tourne la polarisation dans une direction perpendiculaire à $\vec{P}_{0}$ et $\vec{M}(\vec{Q})$ devient non nul. Le sens de rotation est déterminé par la phase relative des amplitudes de diffusion magnétiques et nucléaires et il est opposé pour les 2 types de domaines à 180. L'ensemble de cette situation est résumé dans la figure ci-dessous, avec comme exemples les structures magnétiques de $\alpha \mathrm{Fe}_{2} \mathrm{O}_{3}$ (hématite) et $\mathrm{Cr}_{2} \mathrm{O}_{3}$.

$\mathrm{a}_{2} \mathrm{O}_{3}$

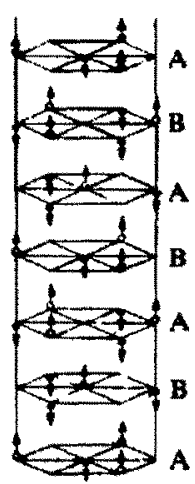

(g)

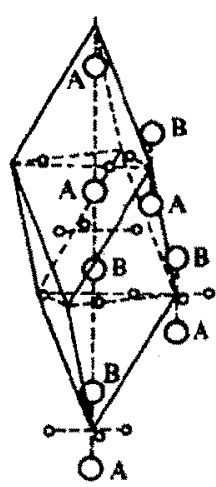

(a)

$\mathrm{AO} \quad \mathrm{BO}$

- Oxygen
$\mathrm{Fe}_{2} \mathrm{O}_{3}$

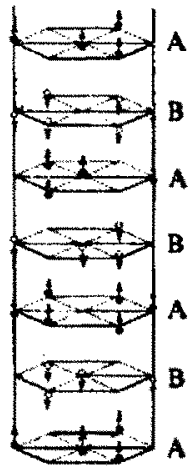

(c)

Fig. 29: Les structures antiferromagnétiques de $\mathrm{Cr}_{2} \mathrm{O}_{3}$ et $\mathrm{\alpha Fe}_{2} \mathrm{O}_{3}$ (hématite). 


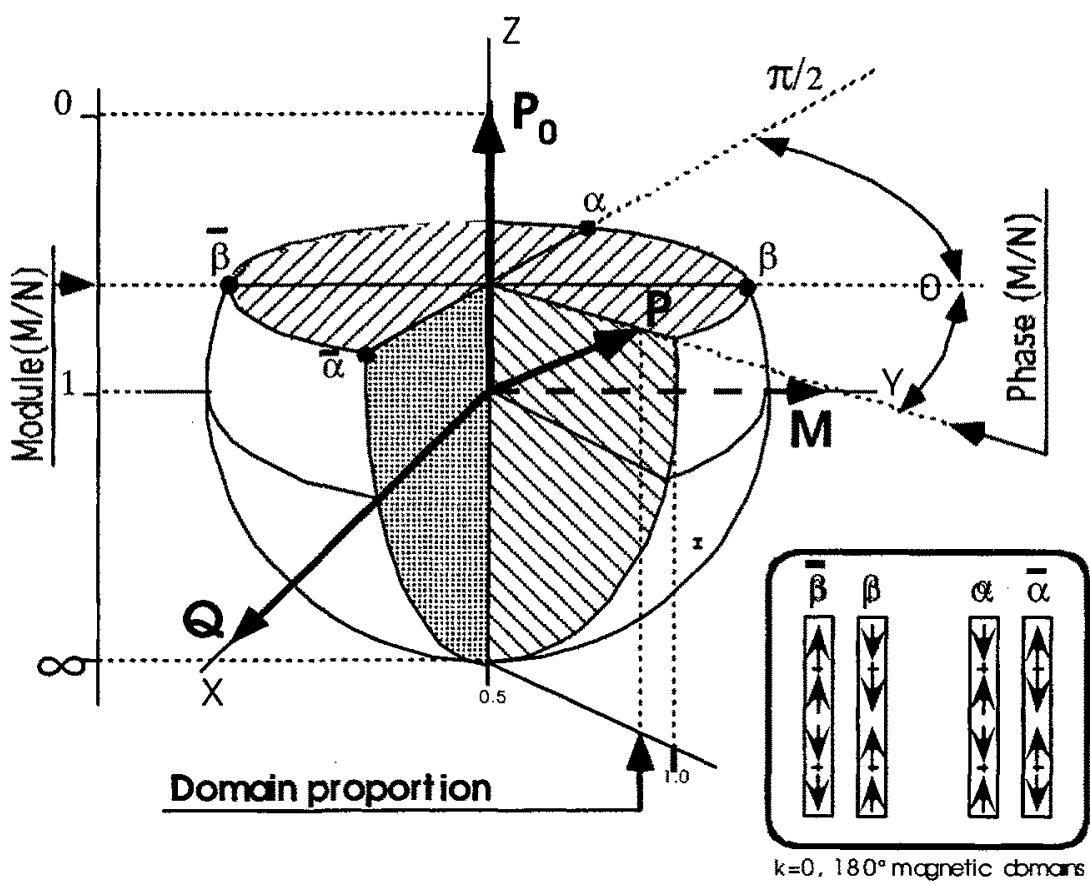

Fig. 30 : Les interactions magnétiques sont impaires dans $\alpha\left(\mathrm{Cr}_{2} \mathrm{O}_{3}\right)$, paires dans $\beta\left(\mathrm{Fe}_{2} \mathrm{O}_{3}\right)$ résultant en différentes rotations pour la polarisation et en une dépolarisation due à la présence des 2 domaines. On peut imaginer quelle sorte de rotation résulterait d'un mélange des 2 types.

\subsubsection{3- Domaines d'orientation}

Les domaines d'orientation se produisent dès qu'une structure magnétique ne peut pas être décrite par un groupe d'espace magnétique congruent avec celui qui décrit la symétrie de configuration. Par exemple, si la symétrie de configuration contient un axe de symétrie d'ordre supérieur à 2 , soit les moments sont parallèles à cet axe, soit la structure est non colinéaire, soit l'axe de symétrie a disparu dans le groupe d'espace magnétique. De manière similaire, dans une structure colinéaire, les moments doivent toujours être parallèles ou perpendiculaires aux plans miroirs et aux axes binaires. En général, le groupe d'espace magnétique $M$ est congruent avec un sous-groupe $S$ de la symétrie de configuration $\mathrm{G}$ et $\mathrm{G}=\mathrm{M} \otimes \mathrm{S}$. Le sous-groupe est constitué des opérateurs contenus dans $\mathrm{G}$ qui ne sont pas dans le groupe magnétique. Si le groupe $S$ est d'ordre $s$ alors il y a $s$ orientations possibles qui sont reliées entre elles par les éléments de $S$. Dans ce cas les vecteurs interaction magnétique des réflexions se correspondant par les éléments de $\mathrm{S}$ seront différents :

$$
\vec{M}(\vec{Q}) \neq \vec{M}\left(R_{s} \vec{Q}\right) \text { mais } \vec{M}_{s}(\vec{Q})=\vec{M}\left(R_{s} \vec{Q}\right)
$$

où $R_{s}$ est un opérateur de $S$ et $\vec{M}_{s}$ est le vecteur interaction pour le domaine généré par $R_{s}$. Pour les structures colinéaires, les facteurs de structure des réflexions reliées par les éléments de $S$ sont égaux: 


$$
R_{s} \vec{F}_{M}(\vec{Q})=\vec{F}_{M}\left(R_{s} \vec{Q}\right)
$$

mais ceci n'est pas vrai dans le cas général.

$\mathrm{La}$ polarisation diffusée par une réflexion purement magnétique avec un vecteur d'interaction magnétique $\vec{M} / / \vec{M}^{*}$ est donnée par eq. (5.10)

Ceci correspond à une précession de la direction de polarisation incidente autour de la direction de $\vec{M}$ par $180^{\circ}$ sans changement de longueur [17] (voir tableau $12(\mathrm{~m})$ ). Si plus d'un domaine est présent dans le cristal, la polarisation finale est la somme des vecteurs de polarisation rendus par chaque domaine pondérée par lintensité due à ce domaine (elle vaut $\alpha_{i} \vec{M}_{i} \vec{M}_{i}^{*}$ où $\alpha_{i}$ est la population relative de domaine i). Considérant le cas de la figure 31 où la polarisation incidente est contenue dans le plan perpendiculaire au vecteur de diffusion et selon l'un des axes qui génère une paire de domaines d'orientation. Les vecteurs interaction magnétique $\vec{M}_{1}$ et $\vec{M}_{2}$ des 2 domaines sont reliés par l'axe de symétrie ( $\mathrm{Y}$ dans la Fig 31 ) et tourne la polarisation rendue dans les directions indiquées par $\vec{P}_{1}$ et $\vec{P}_{2}$ respectivement. Si les populations de domaines sont égales le vecteur de polarisation final est dans la même direction que le vecteur incident, mais de signe contraire si l'angle $\phi$ est supérieur à $45^{\circ}$. Le même type de comportement arrive si le vecteur de polarisation incident est perpendiculaire à l'axe de symétrie, comme indiqué dans la Fig. 31-b

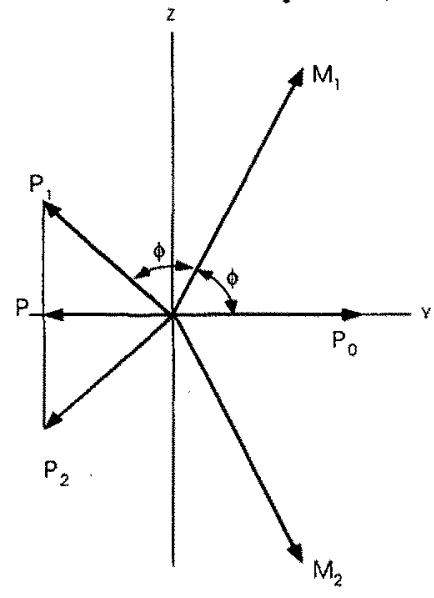

(c)

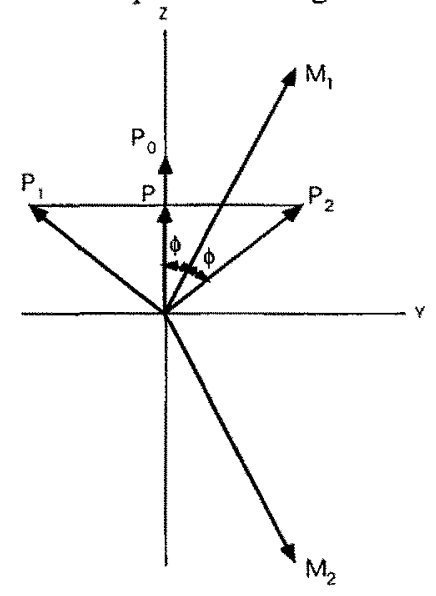

(b)

Fig. 31 : Diagramme montrant la rotation de la direction de polarisation par diffusion dans deux domaines reliés par symétrie avec des vecteurs interaction magnétique $\vec{M}_{1}$ et $\vec{M}_{2}$, La polarisation incidente $\vec{P}_{0}$ est tournée vers $\vec{P}_{1}$ par $\vec{M}_{1}$ et vers $\vec{P}_{2}$ par $\vec{M}_{2}$. La polarisation résultante est la somme pondérée des deux vecteurs. Comme nous supposons que les deux domaines sont en égale proportion la polarisation totale $P_{\text {est }} P_{0} \cos 2 \phi$ où $\phi$ est l'angle entre $\vec{P}_{0}$ et $\vec{M} .(a)$ : La polarisation incidente est perpendiculaire au vecteur de diffusion et parallèle à l'axe de symétrie qui relie $\vec{M}_{1}$ et $\vec{M}_{2}$. (b) : comme pour (a) sauf que la polarisation incidente est perpendiculaire à l'axe de symétrie. 


\subsubsection{4- Domaines de Chiralité}

Les domaines de chiralité arrivent lorsque le groupe d'espace est centrosymétrique mais que la structure magnétique ordonnée ne l'est pas. Le centre de symétrie peut disparaître parce que les moments magnétiques sur les atomes dont les positions sont reliées par des centres de symétrie ne sont pas parallèles ni antiparallèles les uns par rapport aux autres. Une autre possibilité peut correspondre avec une classe spéciale de domaines de configuration : une classe dans laquelle $2 \vec{\kappa}$ n'est pas un vecteur du réseau réciproque $(\vec{K} \neq \vec{\tau}-\vec{K})$, de telle manière qu'il n'y ait plus le centre de symétrie dans le groupe de configuration. Dans ce dernier cas, les deux domaines de chiralité correspondent aux deux valeurs positive et négative de $\bar{\kappa}$; ils donnent tous les deux des réflexions magnétiques à $\vec{g} \pm \vec{\kappa}$ avec

$$
\vec{M}_{\kappa}(\vec{\tau}+\vec{K})=-\vec{M}_{\kappa}^{*}(\vec{\tau}-\vec{K})=-\vec{M}_{-\kappa}^{*}(\vec{\tau}+\vec{K})
$$

Pour chacun de ces arrangements de moments le MrV $\vec{M}$ ne sera pas en général parallèle à $\vec{M}^{*}$ si bien que les termes des Eq. 5.11 et 5.12 respectivement ne sont plus nuls. Le premier de ces termes donne une dépendance de la section efficace avec la polarisation incidente, et le second résulte dans une rotation de la polarisation rendue vers le vecteur de diffusion. Les deux termes changent de signe pour une paire de domaines de chiralité et vont donc s'annuler si les domaines sont peuplés de manière équivalente mais seulement se réduire si les populations sont différentes

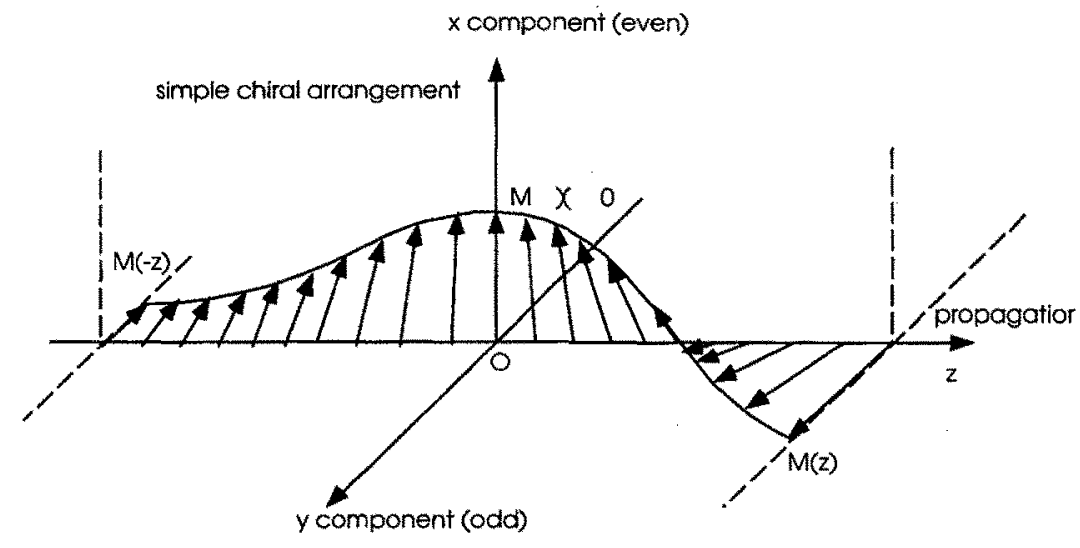

Fig. 32 : Un essai de représentation pour une structure hélicoïdale montrant que la composante $x$ du moment magnétique est une fonction paire de z contrairement a la composante y qui est une fonction impaire. La transformée de Fourier d'une fonction paire étant réelle et celle d'une fonction impaire imaginaire, il en rêsulte que la partie réelle et imaginaire de l'interaction magnétique vont automatiquement pointer dans des directions orthogonales : $\bar{M}=a \hat{x}+i b \hat{y}$. Il en résulte que $\ddot{M} \times \ddot{M}^{*} \neq 0$. 


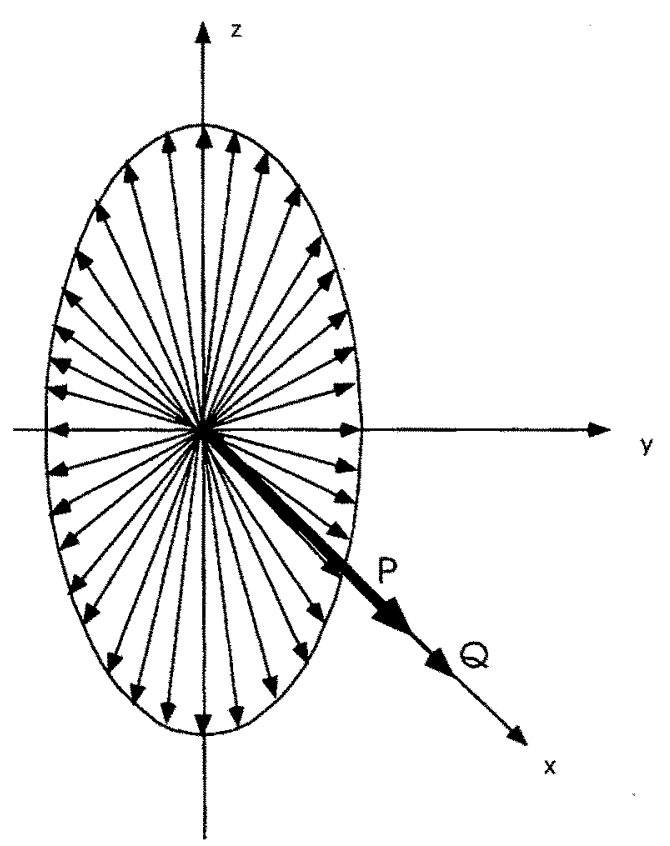

Fig. 33: A cause de la nature dipolaire de son interaction magnétique, le neutron ne voit que la projection de l'hélice sur un plan perpendiculaire au vecteur de diffusion. En fonction de la réflexion observée une hélice parfaitement régulière va donc être perçue comme elliptique ou même comme une modulation sinusoïdale (lorsque $\vec{Q}=\vec{\tau}+\vec{k}$ est contenu dans le "plan de l'hélice"). Comme elle est très influencée par la longueur relative des 2 vecteurs orthogonaux représentant l'interaction magnétique, la mesure de la polarisation finale pour 2 vecteurs de diffusion orthogonaux, lorsqu'elle est possible, est un outil très sensible pour distinguer une hélice d'un arrangement sinusoïdal colinéaire, orienter ses axes principaux et mesurer son ellipticité. (voir par exemple $[2,18])$

\section{2-Facteur de forme antiferro-magnétique \& détermination de la densité magnétique [19]}

Les facteurs de forme des ions magnétiques dans les matériaux ferromagnétiques et paramagnétiques ont été beaucoup étudiés à l'aide des méthodes classiques de rapport de flipping et dans les cas favorables les mesures peuvent être très précises [20]. De tels facteurs de forme sont bien plus difficiles à observer dans les structures antiferromagnétiques parce que, dans ce cas, la section efficace de diffusion ne dépend pas souvent de la polarisation et que la méthode classique n'est donc pas applicable. En conséquence, très peu de facteurs de forme antiferromagnétiques ont été mesurés. Dans les quelques cas où ces mesures ont été réalisées, elles ont donné des résultats très intéressants. Les facteurs de forme antiferromagnétiques sont plus sensibles que les ferromagnétiques ou paramagnétiques à la covalence. C'est parce que la superposition des électrons transférés sur les ions ligands se fait avec des spins contraires résultant en une perte de moment magnétique plutôt qu'une simple redistribution. Jusqu'à présent aucune mesure précise du facteur de forme n'a pu être faite dans des structures antiferromagnétiques où la périodicité des structures nucléaire et magnétique sont les mêmes mais où les atomes de moments magnétiques opposés sont reliés par un centre de symétrie. Dans de telles structures, les réflexions de Bragg d'origine 
nucléaires et magnétique sont superposées ce qui rend difficile la séparation des intensités respectives. En plus les facteurs de structure magnétiques et nucléaires sont en quadrature de phase de telle manière qu'il n'y a pas de partie réelle au terme d'interférence et donc pas de dépendance de la section efficace avec la polarisation incidente. C'est ainsi que la technique nouvelle de Polarimétrie Neutronique Sphérique [14], apte à mesurer la partie imaginaire de l'interférence en question, se révèle apte à des mesures précises de l'amplitude de diffusion magnétique dans de telles structures et que ceci peut servir à la détermination précise des facteurs de forme antiferromagnétique.

Cette technique de polarimétrie que nous avons développée avec Cryopad, consiste dans le choix d'une direction de polarisation initiale et dans la détermination de la direction et du module de la polarisation rendue par l'échantillon pour un vecteur de diffusion et un transfert d'énergie donnés. Dans l'expérience discutée ici nous regardons la diffraction de Bragg élastique.

$\mathrm{Cr}_{2} \mathrm{O}_{3}$ foumit un exemple bien connu d'antiferromagnétisme pour lequel les amplitudes de diffusion magnétique et nucléaires sont non nulles dans les mêmes réflexions de Bragg tout en étant en quadrature de phase

Table 13: Les composantes de polarisation pour $\mathrm{Cr}_{2} \mathrm{O}_{3}$

\begin{tabular}{|l|l|l|}
\hline$P_{x x}=\beta$ & $P_{x y}=0$ & $P_{x z}=\xi$ \\
\hline$P_{y x}=0$ & $P_{y y}=1$ & $P_{y z}=0$ \\
\hline$P_{z x}=-\xi$ & $P_{z y}=0$ & $P_{z z}=\beta$ \\
\hline
\end{tabular}

Il est utile de choisir trois directions orthogonale $(x, y, z)$ pour la polarisation incidente: $z$ est perpendiculaire au plan de diffusion, $x$ est parallèle au vecteur de diffusion, et y complète le système Cartésien de coordonnées (main droite). Les composantes de polarisation rendue paraltèle à ces trois directions peut être représenté par $P_{u x}, P_{u y}, P_{u z}$ ou l'indice u représente la direction de la polarisation incidente $(x, y, z)$ pour cette mesure.

$$
\beta=\left(1-\gamma^{2}\right) /\left(1+\gamma^{2}\right) \text { et } \xi=2 m_{\gamma} \gamma /\left(1+\gamma^{2}\right)
$$

où $\gamma$ est le rapport entre les facteurs de structure magnétiques et nucléaires, et $m_{\gamma}$ la projection de la direction du moment magnétique sur le plan perpendiculaire au vecteur de diffusion. Il y a deux domaines possibles à $180^{\circ}$ pour lesquels $m_{y}$ est de grandeur égale, mais de signe oppose. Si les volumes occupés par les deux domaines dans le cristal sont $v^{+}$et $v^{-}$, on définit l'asymétrie de domaines par:

$$
\eta=\left(v^{+}-v^{-}\right) /\left(v^{+}+v^{-}\right)
$$

et les termes non-diagonaux de la polarisation transverse deviennent

$$
P_{x z}=\eta \xi \quad \text { et } \quad P_{z x}=-\eta \xi
$$

Dans un premier temps, la polarisation du faisceau diffusé pour toutes les réflexions de la forme $h 0 . \ell$ avec $\sin \theta / \lambda\left\langle 0.5 \AA^{-1}\right.$ ont pu être mesurés avec Cryopad-II sur IN20 [21]. Ces mesures, effectuées avec 2 cristaux de tailles différentes et pour trois degrés différents de déséquilibre des domaines, donnèrent des résultats extraordinairement cohérents qui permirent de déterminer avec 
une haute précision la partie aux bas angles du facteur de forme du Cr3+ montré dans la Fig 34 . Cependant il ne fut pas possible d'étendre les mesures à des valeurs plus élevées de $\sin \theta / \lambda$ sur IN20 puisque la valeur maximale du vecteur d'onde incident ne peut dépasser $4.1 \AA^{-1}$.

Nous avons pu prolonger ces mesures pour des vecteurs d'onde plus grands en installant Cryopad-II sur le diffractomètre à neutron polarisés D3 installé sur la source chaude en utilisant le filtre polarisant à 3 He pour analyser la polarisation du faisceau diffracté rendu. Aux valeurs élevées de $\sin \theta / \lambda$ la diffusion magnétique devient très faible et il serait dispendieux de vouloir procéder à toutes les mesures d'analyse de polarisation; mais si le rapport de domaine déterminé avec des réflexions à bas angles est élevé, il est suffisant de mesurer uniquement les composantes $P_{x z}$ et $P_{z x}$ de la polarisation rendue. Celles-ci dépendent linéairement (et non quadratiquement) de $\gamma$. On calibre les efficacités finies du polariseur et de l'analyseur en mesurant la composante $P_{y y}$ qui conserve la polarisation. En utilisant cette méthode nous avons pu déduire les facteurs de structure magnétiques de 14 réflexions $h 0, \ell$ avec un $\sin \theta / \lambda$ compris entre 0.5 et $0.75 \AA^{-1}$. Leur contribution au facteur de forme $\mathrm{Cr}^{3+}$ est montrée dans la Fig 34 et agrandit dans l'inclusion.

Nous avons pu ajuster la partie à bas angle avec le facteur de forme calculé de l'ion libre $\mathrm{Cr}^{3+}$ en prenant un moment de $2.5 \mu_{B}$ pour le chrome. Cette valeur plutôt faible suggère qu'il y ait un transfert covalent significatif vers les oxygènes. Ces points à bas angle sont en bon accord avec une courbe régulière. Les points à plus grands angles sont dispersés de part et d'autre de la courbe représentant une répartition sphérique du moment, cette dispersion contient l'information correspondant aux écarts avec la symétrie sphérique et peut être utilisée pour déterminer la distribution des électrons non-appariés sur les différentes orbitales $3 \mathrm{~d}$.

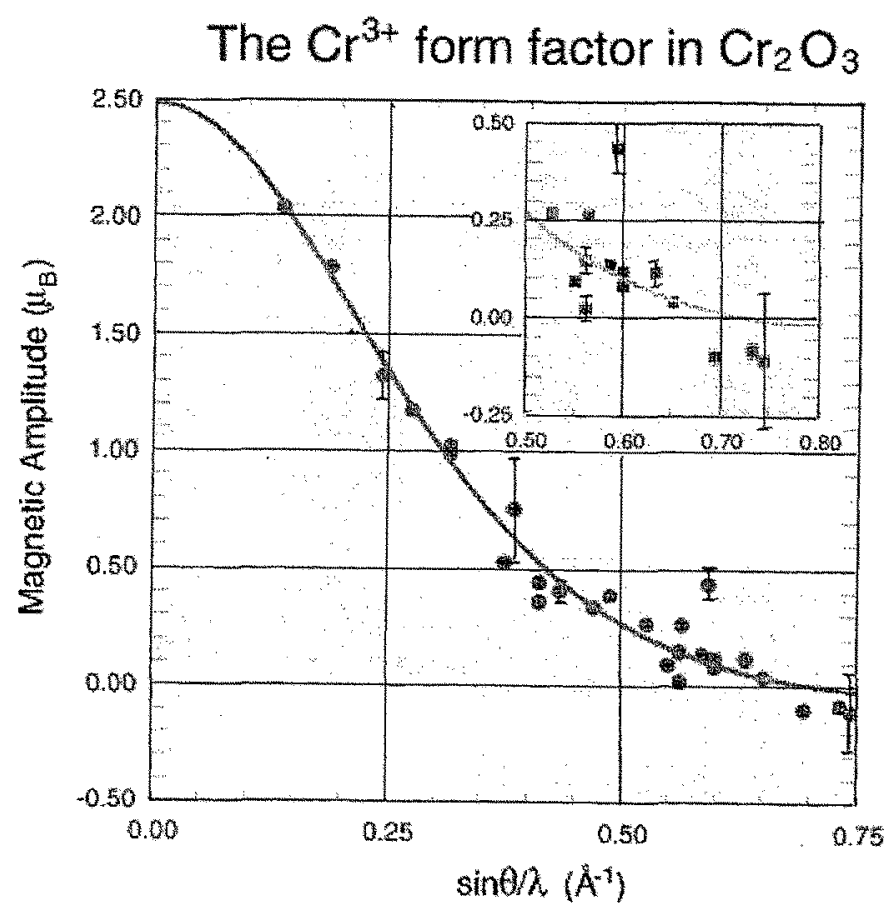

Fig. 34 : les valeurs expérimentales du facteur de forme magnétique mesurées pour les réflexions de Bragg ${ }^{h 0} \mathrm{l}_{\ell}$ de $\mathrm{Cr}_{2} \mathrm{O}_{3}$. La courbe régulière est le facteur de forme spin-pur pour l'ion libre $\mathrm{Cr}^{3+}$ normalisé à $2.5 \mu_{B}$. 


\subsection{Théorie de l'interférence Nucléaire-Magnétique pour la diffusion inélastique des neutrons polarisés}

Très récemment, Maleyev a revisité ce point théorique en détail. Nous reproduisons ici le résumé de son papier [22] suivi par une table des formules clés afin de comparer ses notations et les nôtres.

Le problème des termes d'interférence nucléaire-magnétique (INMI) dans la diffusion (dispersion) des neutrons polarisés est considérée du point de vue théorique. Les expressions générales pour cette interférence sont déduites et discutées. Nous montrons que le INMI apparaît en présence d'un certain type d'interactions qui connectent les variables dynamiques nucléaires et magnétiques et contribue donc au deux parties nucléaire et magnétique de la diffusion. Cependant, à $\vec{H}=0$, ou $\mathrm{H}$ est le champ magnétique appliqué ou l'aimantation de l'échantillon, le INMI s'établit si l'interaction spin-réseau est caractérisée par un vecteur axial. L'exemple de l'interaction de DzialoshinskiiMoriya est considéré. On montre que, dans ce cas, l'INMI est reliée aux termes non diagonaux de la susceptibilité à trois spins qui mettent en jeu des opérateurs non locaux de spin. L'application de cette théorie au composé spin-Peierls $\mathrm{CuGeO}_{3}$ est discutée sur la base de résultats expérimentaux préliminaires.

Les notations utilisées dans notre tableau sont basées sur celles de Maleyev :

En un point $Q$ de l'espace réciproque, l'amplitude de diffusion des neutrons est

$$
F_{Q}=N_{Q}+\vec{M}_{Q} \cdot \vec{\sigma}
$$

avec

$$
\begin{gathered}
N_{Q}=-N^{-\frac{1}{2}} \sum_{n} b_{n} e^{i \vec{Q} \vec{r}_{m}} \text { et } \vec{M}_{Q}=p \vec{F}_{M \perp}(\vec{Q}) \\
p=0.269510^{-12}\left[\mathrm{~cm} / \mu_{B}\right] \text { et } F_{M \perp}(\vec{Q})=-N^{-\frac{1}{2}} \sum_{m} f_{m}(\vec{Q}) e^{i \vec{Q} \cdot \vec{F}_{m}}\left[\vec{m}_{m}-\left(\hat{Q} \cdot \vec{m}_{m}\right) \hat{Q}\right]
\end{gathered}
$$

$b_{n}$ est la longueur de diffusion nucléaire pour l'atome localisé en $\vec{r}_{n}, \vec{m}_{m}$ le moment localisé en $\mu_{B}$ de l'atome magnétique en position $\vec{r}_{m}$ et $f_{m}(\vec{Q})$ le facteur de forme magnétique normalisé à 1 pour $\mathrm{Q}=0$.

Dans le cas inélastique, la susceptibilité généralisée retardée est utilisée

$$
\langle A, B\rangle_{\omega}=i \int_{-\infty}^{+\infty} d t e^{i^{i \omega x}}\langle[A(t), B(0)]
$$

Elle est partagée en une partie dispersive( $\left.{ }^{*}\right)$ et une partie dissipative (")

$$
\langle A, B\rangle_{\omega}=\langle A, B\rangle_{\omega}^{\prime}+i\langle A, B\rangle_{\omega}
$$

La partie dissipative est alors utilisée pour définir les diverses fonctions de corrélation de Van Hove qui interviennent dans les équation de diffusion inélastique des neutrons :

$$
S_{A B}(\omega)=y\left[\mathbb{R}-e^{\left(-\frac{4}{2}\right)}\right]^{1}\langle A, B\rangle_{\omega}^{\prime \prime}
$$

Les 2 équations principales sont découpées en 4 parties qui sont données dans le tableau suivant 


$$
\begin{gathered}
\sigma_{i}=\sigma_{n}+\sigma_{m}+\sigma_{c}+\sigma_{i} \\
\vec{P} \sigma_{i}=(P \vec{\sigma})_{n}+(P \sigma)_{m}+(P \sigma)_{c}+(P \sigma)_{i}
\end{gathered}
$$

Tableau 14: Un parallèle entre les formules de SNP élastiques et inélastiques (notons que dans la

\begin{tabular}{|c|c|c|}
\hline & Elastique:(Blume 1963)[9] & Inélastique : (Maleyev 1998)[22] \\
\hline & $\begin{array}{l}\text { Voir la section } 5.2 \text { pour les } \\
\text { notations }\end{array}$ & $\begin{array}{l}\text { Fonction de corrélation de Van Hove: } \\
v H C F: S_{A B}(\omega)=1 / \pi\left[1-e^{\left(-\frac{4}{T}\right)}\right]^{1}(A, B\rangle^{\prime} \\
(6.15)\end{array}$ \\
\hline $\begin{array}{l}\text { (n) partie } \\
\text { nucléaire }\end{array}$ & $\begin{array}{l}\sigma_{n}=N N^{*} \\
(P \vec{\sigma})_{n}=\vec{P}_{0} \sigma_{n}(5.8)\end{array}$ & $\begin{array}{l}\sigma_{n}=\left(k_{f} / k_{i}\right) S_{n} \\
(\overrightarrow{P \sigma})_{n}=\vec{P}_{0} \sigma_{n} \\
S_{n} \text { is VHCF for }\left\langle N_{-Q}, N_{Q}\right\rangle_{\omega}\end{array}$ \\
\hline $\begin{array}{l}(\mathrm{m}) \text { partie } \\
\text { magnétique } \\
\text { normale }\end{array}$ & $\begin{array}{c}\sigma_{m}=\vec{M} \cdot \vec{M}^{*}(5.9) \\
(P \sigma)_{m}=-P_{0} \sigma_{m}\left\{\hat{P}_{0}-2\left(\hat{P}_{0} \hat{M}\right) \hat{M}\right. \\
(5.10)\end{array}$ & $\begin{array}{l}\sigma_{m}=\left(k_{f} / k_{i}\right) S_{\alpha \beta} \delta_{\alpha \beta} \\
\begin{array}{l}\left(P_{\alpha} \sigma\right)_{m} \\
=\left(\frac{k_{f}}{k_{i}}\right) P_{0 \beta}\left[\left(S_{\alpha \beta}+S_{\beta_{\alpha}}\right)-\delta_{\alpha \beta} S_{\alpha \beta}\right] \\
(6.20)\end{array} \\
S_{\alpha \beta} \text { is VHCF for }\left\langle M_{-Q}^{\alpha}, M_{Q}^{\beta}\right\rangle_{\omega}^{\prime \prime}\end{array}$ \\
\hline $\begin{array}{l}\text { (c) partie } \\
\text { magnétique } \\
\text { chirale }\end{array}$ & $\begin{array}{c}\sigma_{\varepsilon}=i\left(\vec{M}^{*} \times \vec{M}\right) \vec{P}_{0} \\
(5.11) \\
(P \vec{\sigma})_{c}=-i\left(\vec{M}^{*} \times \vec{M}\right) \\
(5.12)\end{array}$ & $\begin{array}{l}\sigma_{c}=\left(k_{f} / k_{i}\right) i S_{\alpha \beta} \varepsilon_{\alpha \beta \gamma} P_{0 \gamma} \\
\left(P_{\alpha} \sigma\right)_{c}=-\left(k_{f} / k_{i}\right) i \varepsilon_{\alpha \beta \gamma} S_{\beta \gamma} \\
S_{\alpha \beta \text { is VHCF for }}\left\langle M_{-Q}^{\alpha}, M_{Q}^{\beta}\right\rangle_{\omega}^{\prime \prime}\end{array}$ \\
\hline $\begin{array}{l}\text { (i) interférence } \\
\text { nucleaire-mag. }\end{array}$ & $\begin{array}{c}\sigma_{i}=\left(N \vec{M}^{*}+N \vec{M}\right) \cdot \vec{P}_{0} \\
(5.13) \\
\overrightarrow{P \sigma})_{i}=N \vec{M}^{*}+N^{*} \vec{M} \\
+i\left[\left(N \vec{M}^{*}-N^{*} \vec{M}\right) \times \vec{P}_{0}\right] \\
(5.14)\end{array}$ & $\begin{array}{l}\sigma_{i}=\left(k_{f} / k_{i}\right) i \vec{S}_{+} \cdot \vec{P}_{0} \\
\left(\overrightarrow{P \sigma}_{i}=\left(k_{f} / k_{i}\right)\left\{\vec{S}_{+}+i\left[\vec{S}_{-} \times \vec{P}_{o}\right]\right\}\right. \\
(6.26) \\
S_{ \pm} \text {is VHCFfor } \\
\left\langle N_{-Q}, \vec{M}_{Q}\right\rangle_{w \pm}^{\prime \prime}=\left\langle N_{-Q}, \vec{M}_{Q}\right\rangle_{\omega}^{\prime \prime} \pm\left\langle\vec{M}_{-Q}, N_{Q}\right\rangle_{w}^{\prime \prime} \\
(6.27)\end{array}$ \\
\hline
\end{tabular}
table originale [4] s'est glissée une erreur de notation dans la formule 4.20 qui est corrigée ici). 


\section{Conclusion}

La diffusion magnétique des neutrons reste un outil de première classe pour l'étude de l'ordre magnétique et des interactions. La diffusion sur poudre a fait de grands progrès grâce à l'introduction des grands détecteurs à localisation combinés avec des optiques optimisées. Les études sur monocristaux sont en pleine êvolution avec l'introduction récente de la méthode QuasiLaue basée sur l'utilisation des plaques photos (image plates) lue par un faisceau laser. On explore ainsi lespace réciproque afin de détecter rapidement les pics de la section efficace de diffusion et le module du vecteur interaction magnétique. Basé sur l'anisotropie dipolaire de son interaction magnétique le neutron peut aussi indiquer la direction des moments magnétiques au travers des extinctions observées et de l'étude systématique des intensités de diffraction mesurées en plusieurs points $\vec{Q}$ différents de l'espace réciproque de Fourier.

En un point $\vec{Q}$ particulier, lutilisation de la Polarimétrie neutronique Sphérique (SNP), peut vérifier, souvent directement, quelle est la direction du vecteur interaction magnétique dans le plan perpendiculaire au vecteur de diffusion. Ceci s'e révèle une méthode puissante, complémentaire des mesures classiques de section efficaces, dans les nombreux cas où elles ne sont pas suffisamment sensibles aux détails de la structure magnétique. (Souvent dû à la coexistence de différents domaines il y a plusieurs modèles possibles pour rendre compte d'un jeu limité d'intensités). De plus, dans de telles situations difficiles, SNP nous conduit à une appréciation plus objective des limites du neutron comme outil d'observation d'une structure antiferromagnétique complexe (arrangements triangulaires ou hélicoïdaux).

Ce qui est le plus spectaculaire avec la diffusion des neutrons polarisés c'est l'exploitation possible du terme d'interférence nucléaire-magnétique qui permet une grande sensibilité pour la mesure des amplitudes magnétiques très petites. Il a été largement utilisé pour la détermination des facteurs de forme magnétiques, c'est-à-dire l'extension spatiale des ions magnétiques, sachant que l'anisotropie des amplitudes de diffusion permet de voir la forme de l'ion magnétique et donc de déterminer son état fondamental à basse température. Nous avons pu montrer récemment que la SNP peut réaliser une telle mesure avec une grande précision dans des arrangements antiferromagnétiques favorables tels que ceux qui donnent lieu à la magnéto-electricité.

Mais l'application la plus passionnante est l'étude des composantes transverses de polarisation dans le domaine de la diffusion inélastique des neutrons; En effet, poussé par des premiers résultats expérimentaux obtenus avec Cryopad-II sur le Cuivre-Germanate, une analyse théorique de la situation a montré que la mesure de la polarisation transverse donne directement accès aux fonctions de corrélation de paires mixtes nuclaire-magnétiques. De telles mesures sont en cours sur plusieurs composés magnétiques présentant des propriétés magnétiques spéciales, spécifiques des basses dimensionalités, avec des excitations de spin non nul dans un rayon fini. Les mesures de polarimétrie neutronique ont le potentiel de détecter le caractère hybride des excitations qui est de plus en plus souvent évoqué dans les modèles électroniques utilisés en physique microscopique à basse dimensionnalité, un domaine très vivant de la matière condensée. 


\section{Annexes: [23]}

\section{A1. Le faisceau de neutrons polarisé}

\section{A.1.1. Aspects quantiques}

Comme le neutron est une particule de spin $1 / 2$, la description quantique de son état dynamique $\mid \Psi>$ nécessite deux composantes $(t,-)$. En terme de fonctions d'onde dans l'espace réel nous aurons

$$
\psi_{+}(\vec{r})=\langle\vec{r},+\mid \psi\rangle \text { et } \psi_{-}(\vec{r})=\langle\vec{r},-\mid \psi\rangle
$$

qui sont généralement groupées en un spineur à deux composantes:

$$
[\psi(\vec{r})]=\left(\begin{array}{l}
\psi_{+}(\vec{r}) \\
\psi_{-}(\vec{r})
\end{array}\right)
$$

Ces deux fonctions des variables d'espace peuvent être complètement différentes si le hamiltonien contient des termes de couplages importants entres les variables orbitales $(\vec{r})$ et les coordonnés de $\operatorname{spin}(t,-)$.

Un exemple très connu est celui de l'expérience de Stern et Gerlach dans laquelle un champ magnétique présentant un gradient important produit deux trajectoires distinctes pour des atomes neutres paramagnétiques d'argent selon l'état de spin dans lequel ils se placent à l'entrée dans le champ magnétique. Un telle expérience ne pouvait pas se faire directement sur un faisceau d'électrons du fait de leur charge électrique q et de la force de Laplace qui en résulte et couple la variable orbitale $v$ directement avec le champ $\mathbf{B}$.

Pour le neutron $q=0$, la force de Laplace s'annule et nous pouvons utiliser l'expérience de SternGerlach pour faire la démonstration de la quantification $1 / 2$ entière de son moment angulaire. Néanmoins, nous verrons plus tard que des champs à évolution lente sont souvent utilisées sur les faisceaux de neutrons sans aucune mention de cet effet. Le moment magnétique du neutron est 3 ordres de grandeur inférieur à celui de l'électron:

$$
\begin{gathered}
\mu_{\text {neut. }}=g_{n} \mu_{0}=-1.913 \frac{m_{e}}{M_{p}} \mu_{B} \\
\mu_{B}=9.2741 \quad 10^{-24} J T^{-1}(S I) \\
\mu_{n e u t .}=9.6622 \quad 10^{-27} J T^{-1}(S I)
\end{gathered}
$$

La déviation Stern-Gerlach est si petite que nous allons pouvoir écrire en première approximation le spineur du neutron comme le produit d'une seule fonction d'onde spatiale par un vecteur état de spin $1 / 2$ :

$$
[\psi(\vec{r})]=\varphi(\vec{r}) \cdot[\chi]
$$


Dans tous les aspects expérimentaux qui seront discutés dans la section 1, ce qui arrive au champ magnétique est à une échelle de longueur plusieurs ordre de grandeur supérieure à la longueur d'onde du neutron. Par suite on peut considérer la position du neutron comme une variable d'espace classique ponctuelle [24] et le moment magnétique du neutron ne va ressentir le champ magnétique qu'en ce point. Dès lors, le temps et l'espace sont simplement couplés par la vitesse classique du neutron et les inhomogénéités magnétiques ressenties sur sa trajectoire vont apparaitre dans l'espace de spin comme de simples quantités dépendant du temps.

L'évolution du vecteur état de spin $1 / 2$,

$$
|\chi\rangle=e^{-i \varphi / 2} \cos \frac{\theta}{2}|+\rangle+e^{i \varphi / 2} \sin \frac{\theta}{2}|-\rangle
$$

sera gouvernée par l'habituelle équation de Schrödinger dépendant du temps. $\theta$ et $\varphi$ vont apparaitre comme des fonctions du temps. (Comme d'habitude elles donnent les angles polaires pour la direction propre de quantification, c'est à dire la direction dans laquelle la composante de spin $S_{z}$ vaut $+\frac{1}{2}$, qui est aussi la direction du vecteur décrivant le moment angulaire classique.)

Les 3 composantes du vecteur opérateur $\vec{S}$ représentant le moment angulaire s'expriment simplement dans la base $|+\rangle,|-\rangle$ par les 3 matrices de Pauli::

$$
\begin{gathered}
\sigma_{x}=\left[\begin{array}{ll}
0 & 1 \\
1 & 0
\end{array}\right], \quad \sigma_{y}=\left[\begin{array}{cc}
0 & -i \\
i & 0
\end{array}\right], \quad \sigma_{z}=\left[\begin{array}{cc}
1 & 0 \\
0 & -1
\end{array}\right] \\
S_{\alpha}=\frac{\hbar}{2}\left\langle\chi\left|\sigma_{\alpha}\right| \chi\right\rangle . \\
\left\langle S_{x}\right\rangle=\frac{\hbar}{2} \sin \theta \cos \varphi,\left\langle S_{y}\right\rangle=\frac{\hbar}{2} \sin \theta \sin \varphi,\left\langle S_{z}\right\rangle=\frac{\hbar}{2} \cos \theta
\end{gathered}
$$

Il faut noter que n'importe quel opérateur hermitique peut aussi s'exprimer dans cette base comme une combinaison linéaire de la matrice unité et des 3 matrices de Pauli.

\section{A.1.2 Moyenne statistique}

Du fait que le faisceau contient de nombreux neutrons qui ne sont pas nécessairement dans le même état de spin, nous définirons la polarisation du faisceau par une moyenne d'ensemble du moment angulaire

$$
\vec{P}=\langle\chi|\hat{\sigma}| \chi\rangle
$$

Un calcul très intéressant est celui de l'évolution de ce vecteur polarisation $\vec{P}$ dans un champ magnétique homogène $\vec{H}$.

Nous partons de l'équation d'évolution des valeurs moyennes quantiques:

$$
\frac{\partial \vec{P}}{\partial t}=\frac{\partial\langle\hat{\sigma}\rangle}{\partial t}=\frac{1}{\hbar}\left\langle\left[\hat{\sigma}, H_{S}\right\rangle\right.
$$

avec le terme d'énergie Zeeman approprié, 


$$
H_{s}=-\hat{\mu} \cdot \vec{H}=-\gamma \frac{\hbar}{2}(\hat{\sigma} \cdot \vec{H})
$$

nous trouvons:

$$
\frac{\partial \vec{P}}{\partial t}=-\frac{\gamma}{2}\left\langle[\hat{\sigma},(\hat{\sigma} \cdot \vec{H}])=\frac{1}{2} i \gamma\langle(\vec{H} \wedge(\hat{\sigma} \wedge \hat{\sigma})))\right.
$$

En utilisant la relation de commutation :

$$
\sigma_{x} \sigma_{y}-\sigma_{y} \sigma_{x}=2 i \sigma_{z}
$$

nous obtenons:

$$
\frac{\partial \vec{p}}{\partial t}=-\gamma\langle(\vec{H} \wedge\langle\hat{\sigma}\rangle))
$$

et finalement

$$
\begin{gathered}
\frac{\partial \vec{P}}{\partial t}=-\gamma((\vec{H} \wedge \vec{P})) \\
\text { avec } \gamma=-18324.61 s^{-1} \text { Gauss }^{-1}
\end{gathered}
$$

\section{A.1.3 ... et comportement classique}

Il est intéressant de chercher quel est, selon la mécanique classique, l'équation du mouvement pour un simple moment magnétique $\vec{m}$ en interaction avec un champ magnétique. Nous avons un système de deux couples, le premier est cinématique

$$
\Gamma_{1}=\vec{m} \wedge \vec{H}
$$

le deuxième vient de l'nertie du moment angulaire:

$$
\vec{\Gamma}_{2}=-\frac{\overrightarrow{d j}}{d t}=-\gamma^{-1} \frac{d \vec{m}}{d t}
$$

A l'équilibre dynamique, la somme des deux couples doit s'annuler et nous avons:

$$
\frac{\partial \vec{m}}{\partial t}=-\gamma(\vec{H} \wedge \vec{m})
$$

Nous constatons que cette dérivation classique aboutit à la même équation que la mécanique quantique, s'agissant de valeur moyennes ( $\vec{P}$ et $\vec{m}$ sont de toute évidence des vecteurs proportionnels). En définitive, s'agissant de champs magnétiques macroscopiques nous avons pu séparé les variables de spin de celles d'espace et nous trouvons finalement que la polarisation, définie comme la valeur moyenne d'opérateurs de spin, se comporte de manière classique.

\section{A.1.4. La précession de Larmor...}

En utilisant l'équation dynamique précédente nous pouvons déduire les caractéristiques principales du mouvement dans un champ constant.

Tout d'abord:

$$
\frac{\partial \vec{m}^{2}}{\partial t}=2 \vec{m} \frac{\partial \vec{m}}{\partial t}=2 \vec{m}(\vec{H} \wedge \vec{m})=0
$$

donc $\vec{m}^{2}=$ const. , le module du vecteur polarisation reste constant.

Ensuite 


$$
\vec{H} \cdot \frac{\partial \vec{m}}{\partial t}=-\gamma \cdot \vec{H} \cdot(\vec{H} \wedge \vec{m})=0
$$

ce qui veut dire que $\vec{H} \cdot \vec{m}=$ Const., la projection de la polarisation sur le champ magnétique appliqué reste constant comme indiqué sur la figure 1 .

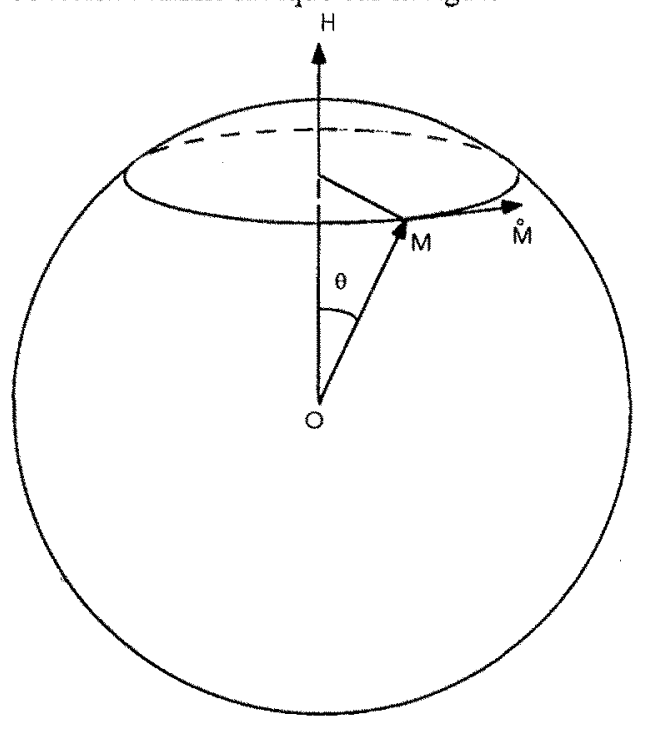

Fig A.I La précession de Larmor du moment magnétique du neutron dans un champ magnétique constant

En définitive

$$
\begin{gathered}
\left|\omega_{L}\right|=\frac{1}{m_{\perp}}\left|\frac{d \vec{m}}{d t \mid}\right|=\frac{\gamma \vec{m} \wedge \vec{H}}{\frac{|\vec{m} \wedge \vec{H}|}{|\vec{H}|}=\gamma H} \\
\omega_{L}=\frac{-1.913 e}{M_{p}} \cdot B(S I) \\
\left|\omega_{L}\right|[\mathrm{rad} / \mathrm{sec}]=18325 \cdot H[\text { Gauss }]
\end{gathered}
$$

ce qui aboutit a une précession lors du déplacement de:

$$
\frac{\Delta \varphi}{\Delta x}[\mathrm{deg} / \mathrm{cm}]=2.65 \lambda[\AA] H[\text { Gauss }]
$$

Un neutron de longueur d'onde $2.4 \AA$ va précesser de $60^{\circ}$ s'il traverse un champ de 1 Gauss pendant $10 \mathrm{~cm}$. 


\section{A.1.5 Réorientation de la polarisation à l'aide d'un "champ de guidage": Paramètre d'adiabaticité}

Nous allons voir plus tard quil est souvent souhaitable de changer la direction de la polarisation du neutron au cours de son trajet dans l'instrument pour satisfaire aux besoins de l'expérience. Ceci est traditionnellement effectué en introduisant des sections de champ de guidage hélicoïdales sur la trajectoire du neutron. La grandeur du champ reste approximativement constante comme dans la Fig. A 2:,

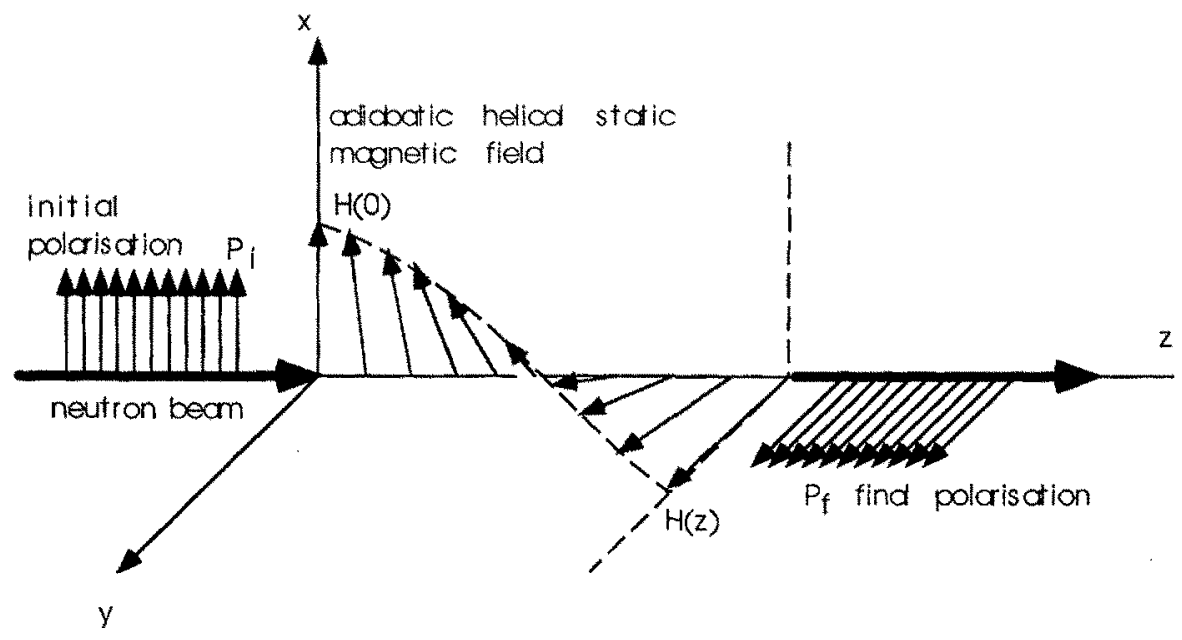

Fig A.2 : Propagation de la polarisation du neutron dans un champ hélicoidal toumant de $\pi / 2$ à module constant. On a supposé ici que le vecteur polarisation suit adiabatiquement la direction du champ de guidage hélicoïdal (voir texte).

En résolvant l'équation de Schrödinger dépendant du temps ou l'équation "classique" dont nous venons de montrer qu'elle lui est équivalente, la polarisation finale a été calculée pour une rotation de $\pi$ [25] ainsi que dans le cas général [26] Le comportement dynamique apparât comme contrôlé par un seul "paramètre d'adiabaticité $\mathrm{E}$ ", le rapport de la vitesse de précession de Larmor et de celle de rotation du champ de guidage (toutes deux peuvent être exprimés en $\mathrm{deg} / \mathrm{cm}$.).

$$
E=\frac{\omega_{L}}{\omega_{H}}
$$

Nous donnons dans la figure 3 les 3 composantes finales de polarisation pour le cas pratique important d'une polarisation initiale parfaite subissant une rotation de champ de $\pi / 2$ 


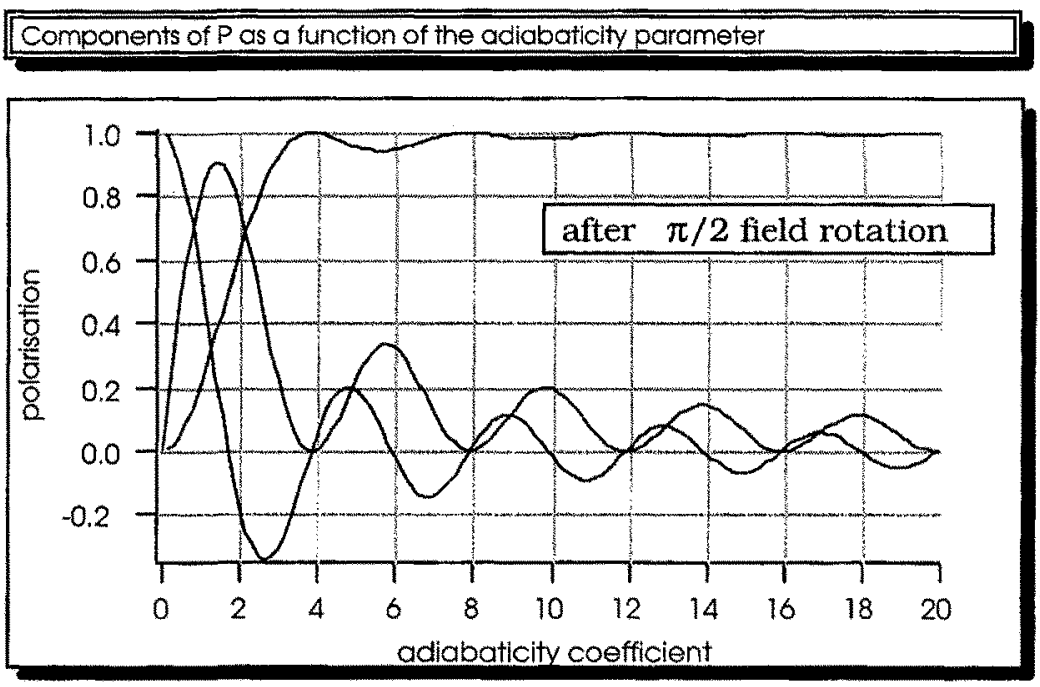

Fig. A.3: Les trois composantes finales de la polarisation du neutron en fonction du paramètre d'adiabaticité à l'issue de son passage dans une configuration de champ de guidage montrée dans la Fig. A.2.

A l'extrême gauche de l'échelle d'adiabaticité, E est très petit, dans le repère du neutron le champ se réoriente rapidement, en moins de temps que la période de Précession de Larmor, le moment de spin du neutron reste dans la direction initiale. Ceci a pour conséquence que la direction du champ magnétique qui était parallèle au moment de spin à l'entrée du système est orthogonale à la sortie. (L'énergie Zeeman est changée et par suite le processus est non-adiabatique pour le neutron). Au contraire, à l'extrême droite de l'échelle la réorientation est lente (Le neutron a fait plusieurs précessions de Larmor pendant le temps passé dans la région de champ hélicoïdal. Nous voyons sur les courbes que deux composantes du moment de spin sont interchangées, de telle sorte que le moment magnétique reste parfaitement aligné avec le champ appliqué. (L'énergie Zeeman est inchangée, le champ a en quelque sorte guidé la composante du spin neutronique qui lui était parallèle et le processus est donc adiabatique pour le neutron).

Pour préciser ce point, nous donnons l'expression analytique de la composante parallèle à la direction finale du champ magnétique en faisant l'hypothèse que la polarisation est selon $x$ à l'entrée:

Pour une rotation de $\pi / 2$ :

$$
\begin{gathered}
P_{y}^{\pi / 2}=1-\frac{2}{1+E^{2}} \operatorname{Sin}^{2}\left(\frac{\pi}{4} \sqrt{1+E^{2}}\right), \\
\text { avec } E=0.03 H[\text { Gauss }] \cdot S[\mathrm{~cm}] \cdot \lambda[\AA]
\end{gathered}
$$

Pour une rotation de $\pi$ : 


$$
\begin{gathered}
P_{x}^{\pi}=-1+\frac{2}{1+E^{2}} \operatorname{Sin}^{2}\left(\frac{\pi}{2} \sqrt{1+E^{2}}\right), \\
\operatorname{avec} E=0.015 H[\text { Gauss }] \cdot S[\mathrm{~cm}] \cdot \lambda[\AA]
\end{gathered}
$$

Le transport de la polarisation par le champ de guidage devient meilleure que $95 \%$ pour les valeurs de E supérieures à $3 ; 98 \%$ pour 8 ; et $99.5 \%$ pour 15 . Cependant nous devons être prudent, lorsqu'elles ont une importance, avec les composantes orthogonales qui ne décroissent que lentement et sont encore de l'ordre de $10 \%$ pour $\mathrm{E}=20$.

Pour finir, nous regardons l'expression de la dérivée par rapport au temps du terme Zeeman.

$$
\frac{d \vec{H} \cdot \vec{P}}{d t}=\vec{H} \cdot \frac{d \vec{P}}{d t}+\vec{P} \frac{d \vec{H}}{d t}=-\gamma \vec{H} \cdot(\vec{H} \wedge \vec{P})+\vec{P} \frac{d \vec{H}}{d t}
$$

Comme le produit mixte dans le terme de droite est toujours nul, il est clair que seul un changement du champ peut changer l'énergie. Si le module du champ de guidage reste constant $\frac{d \vec{H}}{d t}$ est orthogonal à $\vec{H}$ et ne se couple qu'avec les composantes de P qui oscillent à la fréquence de Larmor. Le produit est nul au premier ordre et seuls des termes du second ordre viennent changer l'énergie. C'est là l'origine mécanique du guidage adiabatique.

\section{A.1.6. Passage non-adiabatique à travers une discontinuité de champ magnétique}

Dans certaines situations expérimentales rencontrés en Polarimétrie Neutronique, on doit s'intéresser à ce qui se passe à l'extrême gauche de l'échelle d'adiabaticité. Nous verrons par exemple que pour la mesure de la dépendance de spin de la section efficace des neutrons nous allons devoir retourner (flipper) la polarisation incidente des neutrons afin de mesurer le "flipping ratio".

Parmi les techniques qui ont été utilisées pour accomplir ce retournement une des plus anciennes est connue sous le nom de Feuille de Dabbs, "Dabbs-Foil" en anglais. Le neutron traverse une fine feuille métallique dans laquelle passe un courant électrique intense qui produit un champ magnétique de direction opposée de part et d'autre du plan de la feuille. On pourrait vouloir appliquer les courbes de rotation de champ calculées plus haut avec une rotation de $\pi$ du champ magnétique et dire que le renversement est si brutal qu'on se trouve à $\mathrm{E}=0$. En fait le problème nécessite une discussion différente car le module du champ magnétique n'est pas constant, au contraire il passe par un noud au centre de la feuille.

Ce qui arrive à l'endroit de champ nul va dépendre de manière critique de n'importe quelle petite composante orthogonale parasite présente dans l'environnement. Voici bien longtemps, ce problème a été discuté avec soin dans le contexte d'arrangements Stern-Gerlach successifs pour lesquels de tels difficultés expérimentales se sont présentées [27].

A partir des équations de Majorana appliquées à ce problème il est possible de calculer la polarisation après le renversement du champ: 


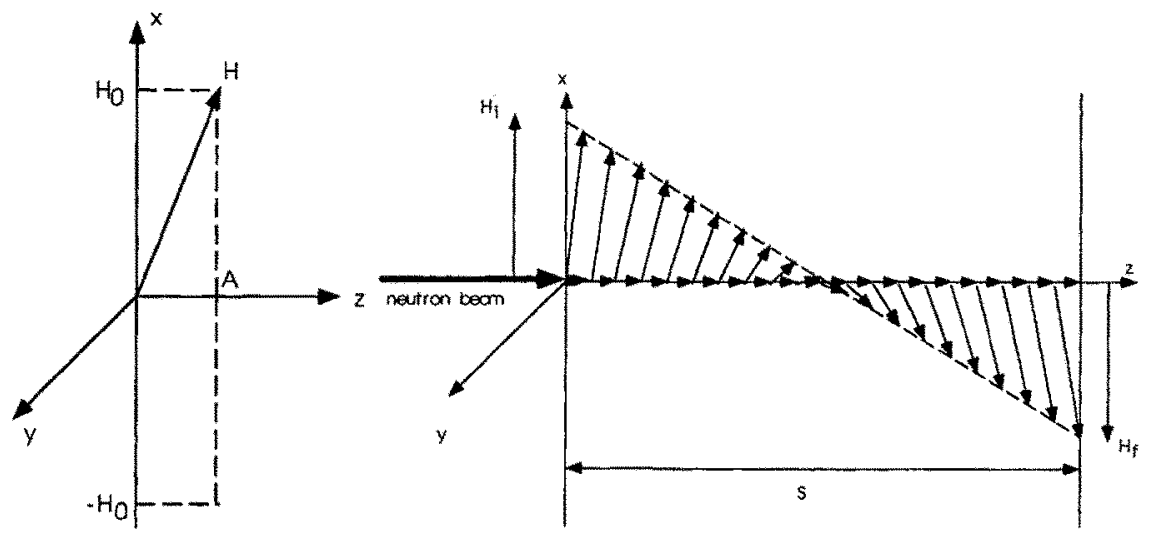

Fig A-4 Composantes de champ magnétique utilisểes dans le calcul du passage non adiabatique à travers une discontinuité de champ

Avec le champ parasite $H_{z}=A$; et le champ principal $H_{y}=0 ; H_{x}=-C t$ et $\mathrm{P}_{\mathrm{f}}=\mathrm{P}_{\mathrm{X}}$ nous trouvons

$$
\frac{P_{f}^{x}}{P_{0}^{x}}=1-2 \exp \left(-k \frac{\pi}{2}\right) \text { avec } k=\frac{g \mu_{N} A^{2}}{\hbar C}
$$

Pour les neutrons thermiques, et en faisant l'approximation au premier ordre, on trouve:

$$
\frac{P_{x}^{f}}{P_{x}^{0}} \cong-1+\frac{0.144 A^{2}\left[\text { Gauss }^{2}\right] \cdot \lambda[A]}{\frac{d y}{d x}[\text { Gauss } / \mathrm{cm}]}
$$

On s'éloigne du retournement idéal d'une manière linéaire en fonction du gradient inverse du champ magnétique principal mais de façon quadratique en fonction du champ parasite. Dans la Fig. 5 nous avons tracé la variation du rapport $\frac{P_{f}}{P_{0}}$ pour plusieurs valeurs plausibles du champ parasite A et du gradient de champ principal en fonction de la longueur d'onde dans le domaine thermique. Nous voyons que pour des neutrons froids et une nappe de courant ordinaire l'écart à la nonadiabaticité idéale peut devenir important. 


\section{Majorana Curves as for Neutron}
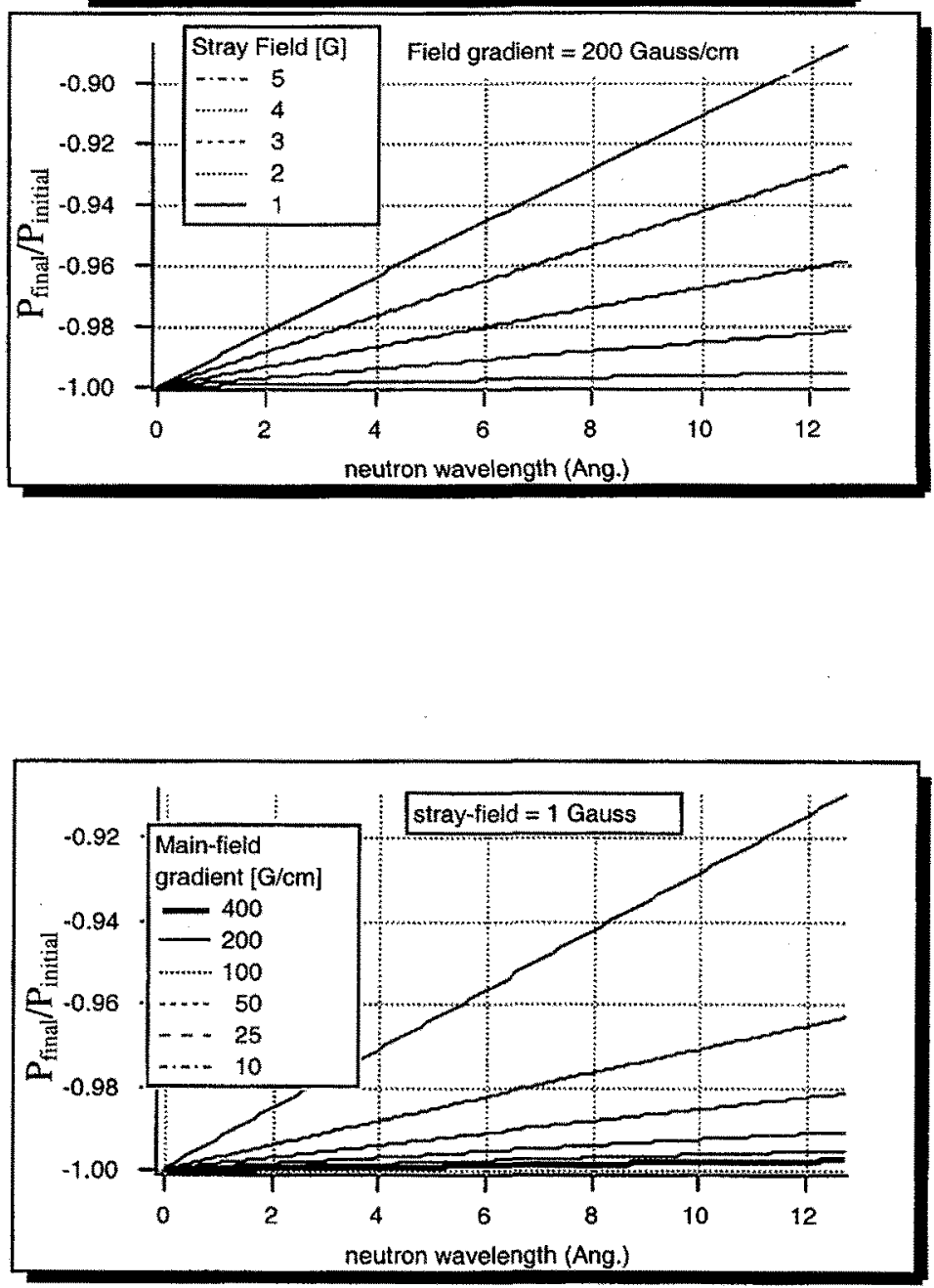

Fig. A.5 Dépendance en longueur d'onde du retournement de la polarisation au passage à travers une "feuille de Dabbs" pour diverses valeurs de la composante parasite constante $A$ et du gradient de champ principal. (N. B. Par suite d'une erreur de graphique il faut diviser l'échelle horizontale par 2 avant lecture). 


\section{A2. Le filtre de spin}

\section{A 2.1. Plus de neutrons polarisés: le filtre de spin à ${ }^{3}$ He polarisé}

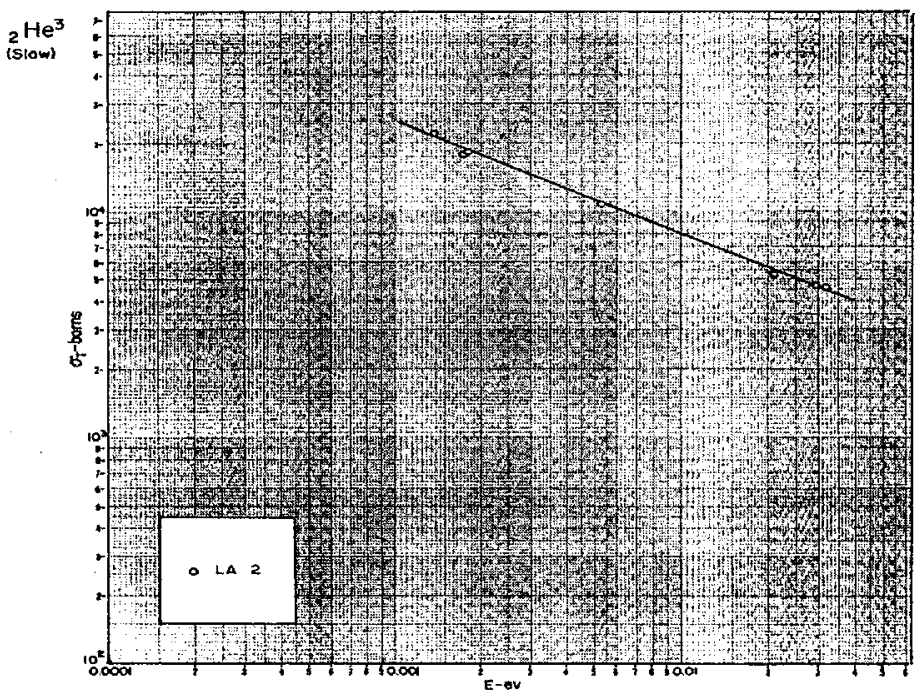

Fig A.6 La section efficace totale de $l^{3}$ He pour les neutrons.

\section{A.2.1.1 Découplage de l'optique et de la polarisation grâce au filtre en transmission}

La préparation de faisceaux de neutrons chauds polarisés à plus de $95 \%$ avec des énergies allant jusqu'à $500 \mathrm{meV}(\lambda=0.4 \AA$.) a pu se faire jusqu'à présent en utilisant des réflexions de Bragg particulières sur certains cristaux magnétiques. La polarisation résulte du fait que les amplitudes de diffusion magnétique et nucléaires s'annulent l'une l'autre pour un des états propre de spin du neutron. Malheureusement, avec les cristaux actuellement disponibles, les réflexions polarisantes se produisent à une valeur relativement basse du transfert de moment et présentent de faibles facteurs de structure pour des raisons qui sont inhérentes au processus d'annulation [28]. Ceci résulte en général en une réflexivité faible et/ou des conditions de résolution médiocres pour des mesures de neutrons polarisés à haute énergie et grand transfert de moment.

L'absorption sélective par certains noyaux polarisés conduit également à une polarisation du faisceau de neutrons transmis. Elle a l'avantage évident de découpler complètement la sélection en énergie de la sélection de spin, ce qui permet toute liberté d'optimiser les conditions de résolution pour le faisceau incident comme pour le faisceau diffusé. 


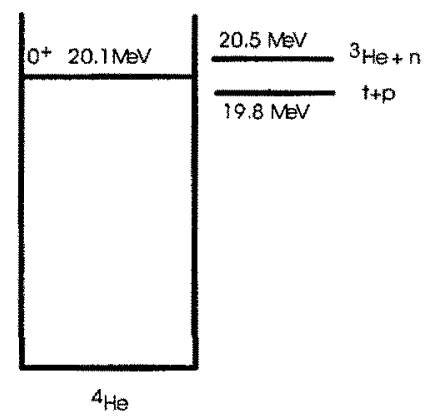

Fig. A.7 Le niveau de ${ }^{4} \mathrm{He}$ correspondant à la résonance responsable de la force et de la dépendance de spin dans la réaction ${ }^{3} \mathrm{He}(n, p) t$

\section{A.2.1.2 Absorption très sélective en Spin}

A priori, l'isotope léger de l'Hélium, ${ }^{3} \mathrm{He}$, peut sembler un candidat étrange pour un dispositif destiné à transmettre des neutron thermiques puisqu'il les absorbe très efficacement dans de nombreux détecteurs de neutrons. De fait, la section efficace de capture des neutrons est énorme et décroît de manière inversement proportionnelle avec la vitesse des neutrons(Fig A.6).

En réalité la mesure de la section efficace de capture pour la réaction ${ }^{3} H e+n \rightarrow t+p$ a montré une forte dépendance de spin [29]. Elle est associée à une résonance large $(270 \mathrm{keV})$ pour ${ }^{3} \mathrm{He}+\mathrm{n}$ qui correspond à un niveau d'énergie $\mathrm{J}=\mathrm{O}^{+}$pour ${ }^{4} \mathrm{He}$ (Fig A.7). En présence d'un absorbeur ${ }^{3} \mathrm{He}$ totalement polarisé, seuls les neutrons de spin antiparallèle à la polarisation de 3 He peuvent subir cette absorption résonante. En pratique on peut espérer atteindre une polarisation de $80 \%$ pour l'3He, si bien qu'avec une épaisseur appropriée, il serait possible de produire un filtre de spin neutronique donnant une polarisation élevée $(95 \%)$ pour une atténuation relativement faible du flux de spin désiré (Fig. A.8) [30-32].

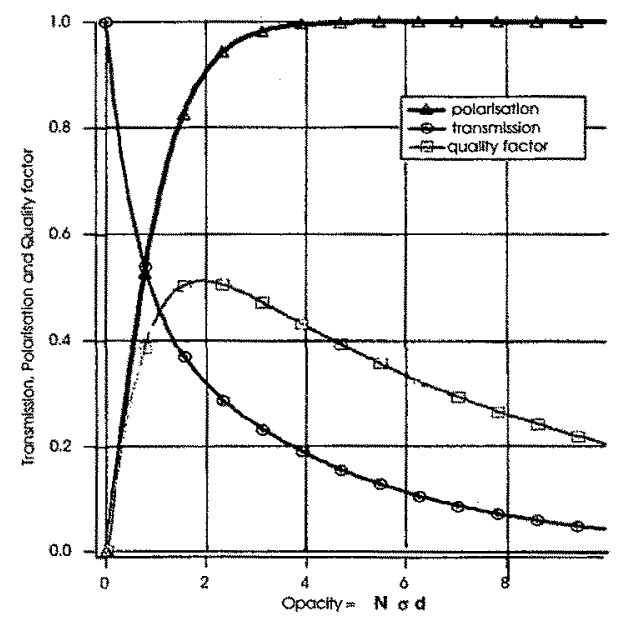

Fig. A.8 Transmission, $T$, polarisation $P$ et facteur de qualité $P T^{1 / 2}$ attendue pour les neutrons en fonction de l'opacitén $e^{\prime \prime} \sigma d$ du filtre pour des noyaux de ${ }^{3}$ He polarisés à $80 \%$. On peut montrer que l'optimum est pour une "opacité" $N \sigma d$ de 2.2 ( $N$ densité atomique, $\sigma$ section efficace d'absorption, $d$ distance traversée) 


\section{Bibliographie}

1. Shull, C.G. and J.S. Smart, MnO. Phys. Rev., 1949. 76: p. 1256.

2. Brown, P.J. and T. Chattopadhyay, The helimagnetic structure of $\operatorname{Eu}(\mathrm{As} 0.2 \mathrm{P} 0.8) 3$

determined by zero-field neutron polarimetry. , Journal of Physics: Condensed Matter, 1997. 9: p. 9167-9173.

3. Tasset, F., Zero field neutron polarimetry. ,Physica B, 1989. 156-157: p. 627-630.

4. Tasset, F., et al., Spherical neutron polarimetry with Cryopad-II. , Physica B, 1999. 267-268: p. $69-74$.

5. Regnault, L.P., et al., Polarized neutron inelastic scattering on the spin-Peierls system CuGeO3: "three-directional" versus "three-dimensional polarization analysis". Physica B, 1999. 267-268: p. 227-235.

6. Nathans, R., et al., The use of polarized neutrons in determining the magnetic scattering by Iron and Nickel., J. Phys. Chem. Solids, 1959. 10: p. 138-146.

7. Brown, P.J. and J.C. Matthewman, The Cambridge Crystallographic Subroutine LibraryMK3, 1987.

8. Schweizer, J., Hercules 1991, Lecture Notes:A .3 Magnetism; Form factors and magnetization densities, MDN/SPh-DRF, CEN Grenoble.

9. Blume, M., Polarization Effects in the magnetic Elastic Scattering of Slow Neutrons. Phys. Rev., 1963. 130: p. 1670-1676.

10. Nunez, $V_{\text {, }}$ et al., Zero field neutron polarimetry. Physica B, 1991. 174: p. 60-65.

11. Brown, P.J., et al., Determination of the magnetic structure of Mn3Sn using generalized neutron polarization analysis. J. Phys. : Cond. Mat., 1990. 2: p. 9409-9422.

12. Moon, R.M., T. Riste, and W. Koehler, Polarization Analysis of Thermal-Neutron Scattering. Phys. Rev., 1969. 181: p. 920-931.

13. Alperin, H. Rotation of the polarization of neutrons scattered from $\mathrm{Cr} 2 \mathrm{O} 3$. in Int. Conf. Magnetism. 1973. Moscow: Proc. ICM-73.

14. Brown, P.J., J.B. Forsyth, and F. Tasset, Neutron polarimetry., Proc. R. Soc. Lond. A, 1993. 442: p. $147-160$.

15. Freund, A.K., Cross-Sections of Materials used as neutron Monochromators and Filters. Nuclear Instruments and Methods, 1983. 213: p. 495-501.

16. Brown, P.J., Magnetic Structure studied with Zero Field Polarimetry. Physica B, 1993. 192(1\&2): p. 14-24.

17. Izyumov, Y.A. and S.V. Maleev, Scattering of polarised neutrons by ferromagnets and antiferromagnets. Sov. Phys. JETP, 1962. 14(5): p. 1168-1171.

18. Brown, P.J., et al., Antiferromagnetism in CuO studied by neutron polarimetry. J. Phys.: Condens. Matter, 1991. 3: p. 4281-4287.

19. Brown, P.J., et al., Determination of the antiferromagnetic form-factor of $\mathrm{Cr} 3+, \mathrm{ILL}$ Annual Report. 1999, Grenoble.

20. Mook, H.A., Magnetic moment distribution of Nickel Metal., Phys. Rev., 1966. 148(2): p. 495.

21. Brown, P.J., J.B. Forsyth, and F. Tasset, Precision measurement of antiferromagnetic form factors. Physica B, 1999. 267-268: p. 215-220.

22. Maleyev, S.V., Nuclear-Magnetic interference at the inelastic scattering of the polarized neutrons. Physica $B, 1999$. 267-268: p. 236-242.

23. Tasset, F., Hercules 1999 -A8 Lecture notes -Neutron Polarimetry, ILL Grenoble. 
24. Halpern, O. and T. Holstein, On the Passage of Neutrons Through Ferromagnets. Phys. Rev., 1941. 59: p. 960-981.

25. Güttinger, P., Das Verhalten von Atomen im Magnetischen Drehfeld. Z. Phys., 1932. 73: p. 169.

26. Newton, R.R. and C. Kittel, On a Proposal for Determining the Thickness of the Transition Layer between Ferromagnetic Domains by a Neutron Polarization Experiment. Phys. Rev., 1948. 74(11): p. 1604-1605.

27. Majorana, E., Atomi orientati in campo magnetico variabile.

Nuovo Cimento, 1932.9 : p. 43.

28. Tasset, F., Report on Allevard discussion for polarisation techniques, . 1989, ILL.

29. Passel, L. and R.I. Schermer, Measurement of the spin dependence of the $3 \mathrm{He}(\mathrm{n}, \mathrm{p}) \mathrm{T}$ Reaction and of the nuclear susceptibility of adsorbed 3He., Phys. Rev., 1966. 150: p. 146.

30. Tasset, F., Towards Helium-3 neutron polarisers. Physica B, 1994. 213\&214: p. 935-938.

31. Tasset, F. and E. Ressouche, Optimum Transmission for an $3 \mathrm{He}$ Neutron Polariser. NIM, A., 1995. 359: p. 537-541.

32. Becker, J., et al., Development of a dense polarized 3 He spin filter based on compression of optically pumped gas. Journal of Neutron Research, 1996. 5(1-2): p. 1-10. 